\title{
WATER USE IN WISCONSIN, 1979
}

\author{
C. L. LAWRENCE AND B. R. ELLEFSON
}

U. S. GEOLOGICAL SURVEY

Water Resources Investigations 82-444

Prepared in cooperation with the

Wisconsin Department of Natural Resources

July 1982 


\title{
UNITED STATES DEPARTMENT OF THE INTERIOR
}

JAMES G. WATT, SECRETARY

\section{GEOLOGICAL SURVEY}

\author{
Dallas L. Peck, Director
}

For additional information write to:

U. S. Geological Survey

1815 University Avenue

Madison, Wisconsin 53706 


\section{CONTENTS}

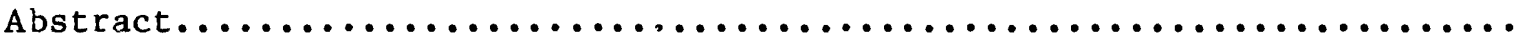

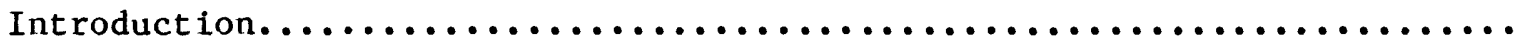

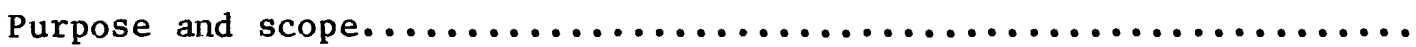

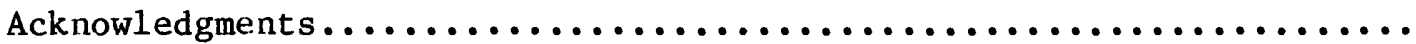

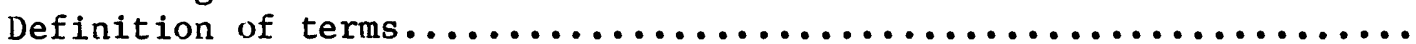

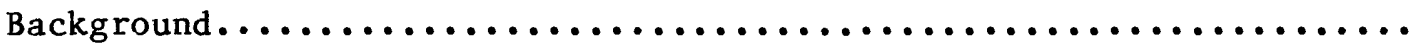

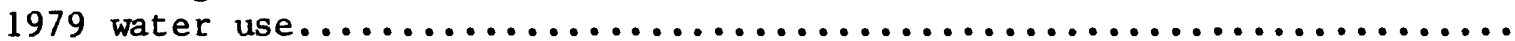

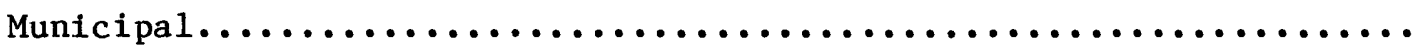

Total withdrawals, excluding hydroelectric and thermoelectric use...

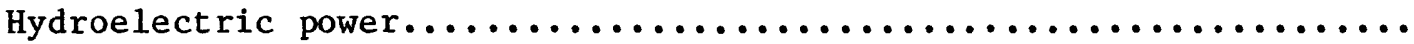

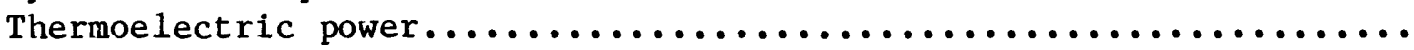

Summary .................................................

References.

\section{ILLUSTRATION}

Figure 1. Map showing location of counties in Wisconsin............

\section{TABLES}

Tables Water use in 1979

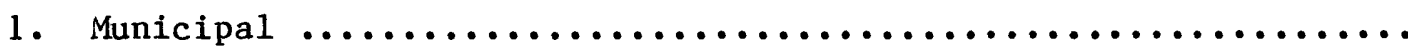

2. Total withdrawal use, excluding hydroelectric and thermo-

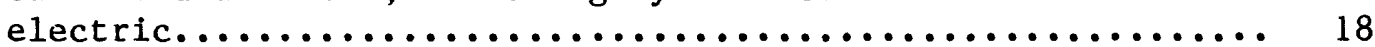

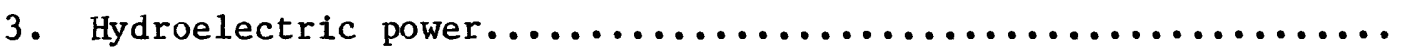

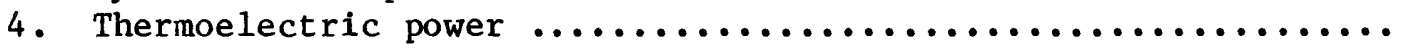

5. Summary for Wisconsin. 


\section{CONVERSION TABLE}

The inch-pound units used in this report may be converted to International System of Units (SI) by the following conversion factors:

Multiply

gallon (gal)

gallon per minute (gal/min)

foot ( $f t$ )

cubic foot per second $\left(\mathrm{ft}^{3 / \mathrm{s}}\right)$
By

3.785

$6.31 \times 10^{-5}$

0.305

0.0283
To obtain

liter (L)

cubic meter per second $\left(\mathrm{m}^{3} / \mathrm{s}\right)$ meter (m)

cubic meter per second $\left(\mathrm{m}^{3} / \mathrm{s}\right)$ 
WATER USE IN WISCONSIN, 1979

By C. L. Lawrence and B. R. Ellefson

\begin{abstract}
This report summarizes the uses of water in Wisconsin for 1979, except aesthetics, navigation, and recreational use.

The greatest single use of water, an instream use, was for hydroelectric power production. About 26 trillion gallons, or 93 percent, was used for this purpose. Of the other 7 percent where water is pumped to a different location, 81 percent ( 6 percent of all water use) was cooling water for thermoelectric powerplants.
\end{abstract}

Withdrawals of surface water and ground water by public and private systems are for residential, industrial, commercial, irrigation, and stock uses.

\title{
INTRODUCTION
}

Wisconsin, by some comparisons, is considered a water-rich State because water is available for almost all present uses. However, as the demands increase, some of the State's water resources w111 be stressed. Information about the use of water is necessary in managing water.

Water-use information is essential for two major purposes: (1) for evaiuating the adequacy of current water sources and (2) for projections of future water needs.

This report tabulates all withdrawals in Wisconsin involving pumping or diverting ground water or surface water from its natural flow pattern. Some typical uses not considered are (1) navigation, (2) recreation, (3) aesthetic considerations, and (4) other purposes than energy production on dammed streams.

\section{PURPOSE AND SCOPE}

A major undocumented factor in many water-resources investigations is the quantity of water actually used for various purposes. The purpose of this report is to document water use in Wisconsin for 1979 .

Although water-use reports have been prepared previously, this report is based on a considerable number of new data sources, different methods of estimating water use, and a classification, by county, of all withdrawals except for thermoelectric power. 
The water-use figures in this report apply only to Wisconsin. Several streams separate Wisconsin from other States. Water for hydroelectric power production along boundary streams is listed only where the generating plant is in Wisconsin. This also is the case for thermoelectric powerplants.

All water-use figures include only fresh water; saline or brackish water use, if any, is not significant.

\section{ACKNOWLEDGEMENTS}

Most of the data in this report were extracted from the files of various State agencies, as mentioned under "Methods". Partial support for the project came from the Department of Natural Resources in the form of personnel service, advice, and use of their facilities. In particular, we acknowledge the help of Tom Calabresa and Robert Devaul of the Department of Natural Resources, who supplied the information on noncommunity public systems and on rural domestic and stock ground-water use. They also supplied much information about nonmunicipal community systems and on private industrial use. The Fox Valley Water Quality Planning Agency provided helpful information on industrial water use in Brown, Outagamie, and Winnebago Counties ( $\mathrm{fig} \cdot 1$ ).

The report was prepared as part of a cooperative program between the Wisconsin Department of Natural Resources and the U.S. Geological Survey. Data collection functions under this program are an element in a National Water Use Data System.

\section{DEFINITION OF TERMS}

Commercial use--Water used for commercial purposes, such as use by motels, retail outlets, restaurants, resorts, and apartment buildings.

Community system--A public water system which has at least 15 service connections used by year-round residents or regularly serves at least 25 year-round residents.

$$
\begin{aligned}
& \text { Ft } 3 / \text { s--Cubic feet per second. } \\
& \text { Gal/d--Gallons per day. } \\
& \text { Gal/min--Gallons per minute. }
\end{aligned}
$$

Industrial use--Water used by industries, such as papermills, canneries, and manufacturing plants.

Irrigation use--Water applied to crops, golf courses, parks, or commercial grounds, but not domestic lawns. 
Kwh--Kilowatt hours.

Municipal system--A community water system owned and operated by an incorporated city or village.

Mwh--Megawatt hours.

Noncommunity system--A public water system having less than 15 service connections used by year-round residents, or serving less than 25 year-round residents.

Nonmunicipal system--A water system not owned or operated by an incorporated city or village.

Other community system--A nonmunicipal water system operated by a town, sanitary district, subdivision, or mobile home park.

Other use--Water uses not specifically categorized, such as use in parks, schools, and public buildings, fire control, water-main flushing and leakage.

Private system--A water system belonging to a particular person or group of persons.

Public system--A water system belonging to, or operated by, the people as a whole or the community at large.

Residential use--Water used for personal services in housing units of not more than two families.

Stock use--Water consumed by domestic farm animals.

\section{BACKGROUND}

Much water-use information for the State has been collected. By State statute, many users are required to report quantities of water withdrawn from ground-water or surface-water bodies and the quantities of surface water returned. Much of these data are in files of State agencies and, although available to the public, have not previously been assembled and published in a readily accessible form. Agencies that provided access to these data for this report include:

1. Wisconsin Department of Natural Resources

2. Wisconsin Public Service Commission

3. Fox Valley Water Quality Planning Agency.

A brief description of the known literature related to water use in Wisconsin follows. 
A thesis written by Harvey E. Wirth (1959) summarizes estimated water use in 1957 and discusses legal aspects of water-use conflicts.

A report, "The Water Use Information Gap--The Wisconsin Experience" (Daniel and Mara, 1975), discusses the needs and uses of water-use information. Suggestions for filling gaps in data needs are given.

Each 5 years the U.S. Geological Survey has published reports summarizing estimated use of water by State and region. The most recent available (Murray and Reeves, 1977) provides estimates for 1975. The data given are useful at the national but not State level.

A publication of the Department of Natural Resources "State of Wisconsin Public Water Supply Data 1970" provides helpful statistical data about municipal wells in existence at the time of its publication.

The Public Service Commission of Wisconsin (PSC) publishes an annual report giving statistical data on power generation that can be related to water use (Wisconsin PSC, 1980).

\section{WATER USE}

Water-use data are summarized in five tables. Tables 1 and 2 summarize water-use data for individual counties. Tables 3, 4, and 5 categorize data in a statewide format. The data were obtained and tabulated as described in the following five sections.

\section{MUNICIPAL WATER USE}

Municipal water use is tabulated by county (fig. 1). The residential, industrial, and commercial categories of water deliveries by municipal systems were taken from files of the Wisconsin Public Service Commission (PSC). All incorporated cities and villages that have centralized water systems are listed. Two towns, Allouez in Brown County and Fitchburg in Dane County, are included because they are extensively urbanized. Cities, villages, and towns are not shown in figure 1. Figures of total water use for the municipal systems are from files of the Public Water Supply Section of the Wisconsin Department of Natural Resources (DNR) and are sums of total pumpage into the system from all sources. The column labeled "other" is the difference between the total pumpage as reported to the DNR and the sum of residential, industrial, and commercial deliveries reported to the PSC. It includes water used for public buildings, firefighting, parks, and transmission losses. Population figures are 1979 estimates supplied by the Wisconsin Department of Administration (1979). 


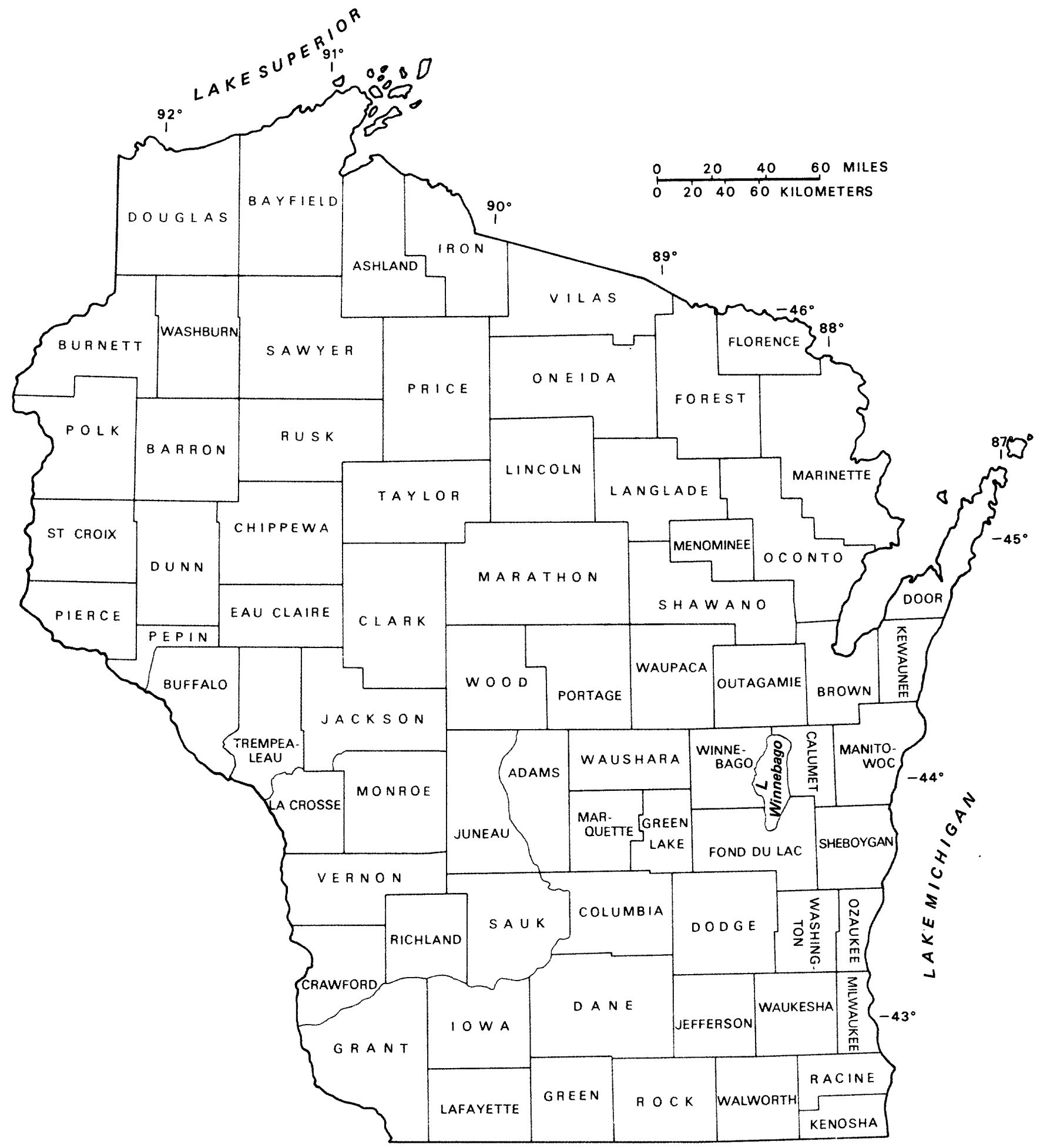

Figure 1. Location of counties in Wisconsin. 
TOTAL WITHDRAWALS, EXCLUDING HYDROELECTRIC

AND THERMOELECTRIC USE

Table 2 includes all uses except that for hydroelectric and thermoelectric power generation. The data are listed by county (fig. 1) and come from several sources.

Under public community systems, figures for municipal water use have been taken from table 1 .

The "other" category includes all nonmunicipal community systems. Most such systems serve townships, sanitary districts or subdivisions, and mobile home parks. The figures are from reports of individual systems to the Wisconsin DNR and PSC. Where Individual reports were not avallable, community use was estimated from population figures applicable to water plants as complled by the DNR and an average consumption figure of $50 \mathrm{gal} / \mathrm{d}$ per person. This figure is based on observations by the Public Water Supply Section of the Wisconsin DNR. It was assumed that all water use by nonreporting systems was residential.

Noncommunity public systems include, but are not limited to, camps, schools, motels, resorts, churches, and parks. They are commonly small users, but are numerous. Typlcally, they draw water from low-capacity (less than $70 \mathrm{gal} / \mathrm{mln}$ ) we11s. Calebresa and Devaul (1981, written commun.) calculated total groundwater use based on a DNR inventory of noncommunity public systems, full operational population, and a use factor to reflect limited seasonal use. A use factor of $13 \mathrm{gal} / \mathrm{d}$ per person was used together with the seasonal use factor, when appropriate. The average per day per person use factor was obtained by analyzing use in five counties having populations between 10,000 and 50,000, estimating per person consumption for each type of system (motel and resort).

Private water systems are those that are self-supplied and that do not normally provide service to the public. Examples are rural homes, farms, and businesses. To illustrate further, a restaurant pumping its own water is considered to be a commercial use from a noncommunity public system, whereas a paper mill is considered to be an industrial use from a private system, provided it supplies its own water.

Calabresa and Devaul (1981, written commun.) provided figures for stock and private residential ground-water use. The residential use was computed by multiplying the average consumption of $50 \mathrm{gal} / \mathrm{d}$ per person times the county population not already considered in community systems. The stock use was computed by multiplying numbers of farm animals (Wisconsin Agriculture Reporting Service, 1979) in counties by standard consumptive values for each type of farm animal; for example, $20 \mathrm{gal} / \mathrm{d}$ for a dalry cow (Todd, 1970).

The industrial, commercial, irrigation, and other categories for private systems were complled from a 1979 DNR summary of annual pumpage of all private high-capacity wells in the State. Pumpage for 1979 for each category was computed based on numbers of wells, and pumpages for those wells with reported pumpage. 
Industrial use of ground water was derived from several sources; the reported high-capacity (greater than $70 \mathrm{gal} / \mathrm{min}$ ) well pumpage, available lowcapacity well pumpage, computed high- and low-capacity well pumpage based on equipment-design criteria and raw materials processed, waste-water discharge permits and waste-discharge volumes from sewer rental lists.

Commercial use of ground water is the sum of reported high-capacity well pumpage, and estimates of unreported pumpage. In estimating unreported pumpage, it was assumed that 9 of 10 high-capacity wells were pumped in 1979 and at 85 percent of their permitted pumpage. This is equivalent to 6 days of operation per week. Most wells used commercially fall into the public noncommunity system category.

Irrigation use of ground water is the sum of the reported pumpage and estimates of unreported pumpage. Estimates for 95 percent of wells with unreported pumpage were made by applying the average rate and time of operation of weils with reported pumpage.

Private-system water use in the "other" category was taken from the previously mentioned DNR summary of annual pumpage of privately owned high-capacity wells. To this was added the flow at State fish hatcheries, which generally use water from flowing wells (Calabresa and Devaul, 1981, written commun.).

Except for municipal systems, all water use discussed thus far for table 2 has been for ground water. Private systems do make some use of surface water. In Wisconsin, surface water is used by industry, by irrigators, and for stock.

Surface-water use by industry is principally by pulp and paper companies. These companies are required to have discharge permits and to report discharges to the Wisconsin DNR. Mills normally measure quantities pumped and assume that it is all discharged to the receiving stream. Tabulations were made from DNR files of average annual flow and numbers of days in operation. The number of gallons pumped from and returned to the stream in 1979 were determined from these tabulations.

Tabulations of surface-water use for irrigation were provided by the six district offices of DNR and coordinated by Robert Roden of DNR. Irrigators are required to have permits to pump from lakes and streams and to report quantities pumped to DNR. Permits are not required in Wisconsin for pumpage from artificial ponds not connected to a surface drainage system. Estimates of these small amounts of use in the form of a percentage of permitted pumpage were made by personnel of the DNR district of fices.

In determining surface-water use by stock each type of animal was not considered because of the small amount of water involved. Surface-water use for all stock was estimated to be 5 percent of the computed ground-water use by cattle alone. This percentage was based on estimates by several county agricultural agents throughout the state. 
The listed values in table 2 do not necessarily add up precisely to the totals because of rounding.

\section{HYDROELECTRIC POWER}

The use of water to generate hydroelectric power is estimated for the plants of all private or municipally owned utilities in the State (table 3 ). Water-use estimates were determined by the formula

$$
\text { MGD }=\frac{1.25 \mathrm{Kwh} / \mathrm{yr}}{1,000 \mathrm{H}},
$$

where: $\quad$ MGD $=$ million gallons per day, $\mathrm{kwh} / \mathrm{yr}=\mathrm{kilowat}$ hours per year, and

$\mathrm{H}=$ difference, in feet, between headwater and tailwater.

This is a standard relation between streamflow, power potential, and net head, assuming 70 percent turbine/generator efficiency. Flow capacity at normal head is the maximum rate of discharge that can flow through a plant at any time. These values and average head values (H) were obtained from the Upper Mississippi River Comprehensive Basin Study (1970) or from the individual plant owners. Annual power generation data were obtained from the Public Service Commission (1980).

\section{THERMOELECTRIC POWER}

The use of water at thermoelectric powerplants also is estimated for all private, municipal, or cooperatively owned utilities in the State (table 4). Surface-water use figures were obtained from DNR's reports of daily coolingwater diversions. The ground-water values listed in this table are from the DNR reports of pumpage for private high-capacity wells. This ground-water pumpage is primarily for make-up water. Any water used for domestic purposes within the plant is insignificant and is not pertinent to this table. Annual power generation data were obtained from the Public Service Commission (1980).

\section{SUMMARY}

Table 5 summarizes water use for the State incorporating data from tables $1-4$.

By far the greatest use of water is for hydroelectric power production. Of the total 1979 use of 28.3 trillion gallons, 26.1 trillion gallons, or 
92 percent, was used for this purpose. This use was not necessarily exclusionary. That is, the same water may be reused downstream for the same or other uses. Privately owned companies operated the dams and powerplants using 23.1 trillion gallons, and the remaining was used by municipally owned plants.

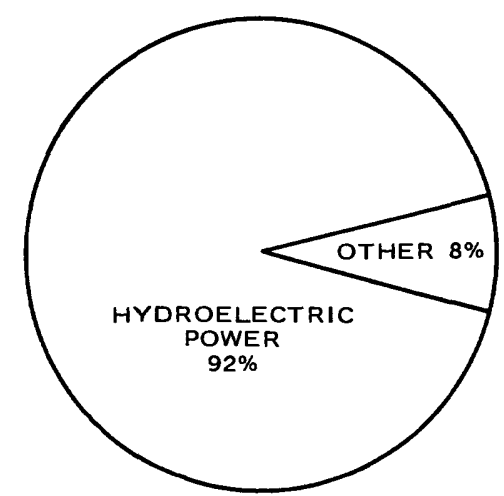

of the remaining 2.15 trillion gallons ( 8 percent of total water use), 1.7 trillion gallons ( 81 percent) were used for cooling water for thermoelectric powerplants. Most of this use was by privately owned companies; the second-greatest user was a rural electric cooperative, entered under "other" as a type of public community systems in table 5 . Almost all this water was pumped from surface sources.

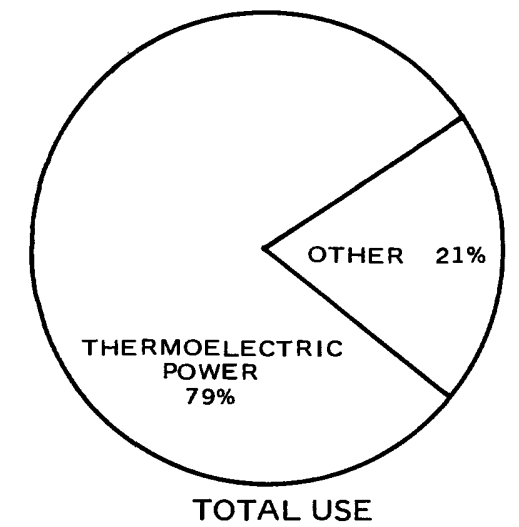

OTHER THAN FOR HYDROELECTRIC POWER

The other 0.45 trillion gallons withdrawn was distributed among the various uses shown in table 5. The largest single type of use for this remainder was as process water at pulp and paper industries, about 0.13 trillion gallons.

The sources of water, other than that for thermoelectric and hydroelectric power, are approximately equally divided between surface water and ground water. However, individual uses may be heavily from one source. For example, irrigation and stock water come mostly from ground water, whereas water used by industry comes principally from surface water.

Water pumped by cities and villages is about equally divided between surface-water and ground-water sources. Ninety-four percent of these municipalities use ground water; however, because several large population centers are located on the shore of Lakes Michigan, Superior, and Winnebago and pump from those lakes, the actual gallonages from surface-water and ground-water sources are similar. At present, with one exception, all communities not on or near the shore of these lakes pump their domestic water supply from underground. The exception is the village of Port Edwards in Wood County, which in 1979 pumped 24 percent of its domestic water supply from a nearby lake. 
The uses of water from municipal utilities were distributed as follows: residential, 32 percent; industrial, 32 percent; commercial, 15 percent; other, 21 percent. The "other" category includes water used for public installations of various kinds (buildings and parks), firesfighting, and for system losses. Water sold by one municipality to another is not considered, except as distributed by the receiving entity.

\section{REFERENCES}

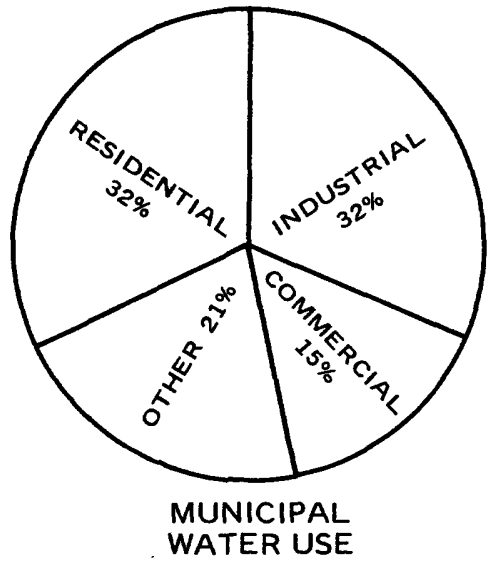

Daniel, D., and Mara, S., 1975, The water use Information gap--the Wisconsin experience: Wisconsin Department of Natural Resources, 124 p.

Murray, C. R., and Reeves, E. B., 1977, Estimated use of water in the United States in 1975: U.S. Geological Survey Circular 765, 39 p.

Public Service Commission of Wisconsin, 1980, Generating plants operated by Wisconsin electric utilities in 1979: Bulletin No. $46,13 \mathrm{p}$.

Todd, D. K., ed., 1970, The water encyclopedia: Water Information Center, p. $275-281$.

Upper Mississippi River Comprehensive Basin Study Coordinating Committee, 1970, Upper Mississippi River comprehensive basin study: Appendix M, Power, 68 p.

Wirth, H. M., 1959, Water use in Wisconsin: Madison, University of Wisconsin, Master's thesis, 113 p.

Wisconsin Agriculture Reporting Service, 1980, Wisconsin agricultural statistics 1979: Publication 200-79, 99 p.

Wisconsin Department of Administration Demographic Services Center, 1979, official population estimates for 1979: $47 \mathrm{p}$.

Wisconsin Department of Natural Resources, 1970, State of Wisconsin public water supply data 1970: $101 \mathrm{p}$. 
Table 1.--Municipal water use in 1979

\begin{tabular}{|c|c|c|c|c|c|c|c|c|}
\hline & & & & & WATER DE & VERIES IN & IONSO & \\
\hline CDINTYY & MUNICIPALITY & SOURCE & POPULATION & RESIDENTIAL & INDUSTRIAL & COMMERCIAL & OTHER & TOTAL \\
\hline ADAMS & $\begin{array}{l}\text { ADAMS CITY OF } \\
\text { FRIENNSHIP VILLLAGE OF } \\
\text { TOTAL }\end{array}$ & $\begin{array}{l}\text { GW } \\
\text { GW }\end{array}$ & $\begin{array}{r}1510 \\
818 \\
2328\end{array}$ & $\begin{array}{l}26.60 \\
10.20 \\
36.80\end{array}$ & $\begin{array}{l}21.90 \\
4.16 \\
26.06\end{array}$ & $\begin{array}{l}12.20 \\
14.80 \\
27.00\end{array}$ & $\begin{array}{r}7.66 \\
5.66 \\
13.32\end{array}$ & $\begin{array}{c}68.36 " \\
34.82 \\
103.18\end{array}$ \\
\hline ASHLAND & $\begin{array}{l}\text { ASHLANO CITY OF } \\
\text { BUTTERNUT VILLAGE OF } \\
\text { MELLEN CITY OF } \\
\text { TOTAL }\end{array}$ & $\begin{array}{l}\text { SW } \\
\text { GW } \\
\text { GW }\end{array}$ & $\begin{array}{r}9768 \\
477 \\
1033 \\
10278\end{array}$ & $\begin{array}{r}135.04 \\
6.01 \\
13.10 \\
154.15\end{array}$ & $\begin{array}{l}0.0 \\
4.67 \\
15.80 \\
20.47\end{array}$ & $\begin{array}{r}147.81 \\
1.52 \\
4.22 \\
153.55\end{array}$ & $\begin{array}{r}151.87 \\
27.95 \\
37.37 \\
192.19\end{array}$ & $\begin{array}{r}434.72 \\
15.15 \\
70.49 \\
520.36\end{array}$ \\
\hline RARRON & $\begin{array}{l}\text { ALMENA VILLAGE OF } \\
\text { AARRON CITY OF } \\
\text { CAMERON VILLAGE OF } \\
\text { CHETEK CITY OF } \\
\text { CUMBERLAND CITY OF } \\
\text { DALLAS VILLAGE OF } \\
\text { HAUGEN VILLAGE OF } \\
\text { RICE LAKE CITY OF } \\
\text { TURTLF LAKE VILLAGE OF } \\
\text { TOTAL }\end{array}$ & $\begin{array}{l}G W \\
G W \\
G W \\
G W \\
G W \\
G W \\
G W \\
G W \\
G W\end{array}$ & $\begin{array}{r}479 \\
2420 \\
1168 \\
1721 \\
1986 \\
393 \\
248 \\
7860 \\
714 \\
16989\end{array}$ & $\begin{array}{r}7.72 \\
39.90 \\
18.06 \\
26.74 \\
33.19 \\
6.55 \\
2.63 \\
106.00 \\
10.31 \\
251.10\end{array}$ & $\begin{array}{r}0.0 \\
168.00 \\
0.0 \\
1.26 \\
97.27 \\
0.0 \\
0.97 \\
48.40 \\
5.91 \\
321.80\end{array}$ & $\begin{array}{r}3.12 \\
117.00 \\
8.58 \\
20.50 \\
22.41 \\
2.75 \\
0.14 \\
143.00 \\
4.61 \\
322.11\end{array}$ & $\begin{array}{r}6.93 \\
36.14 \\
47.23 \\
67.85 \\
63.07 \\
3.00 \\
2.30 \\
223.20 \\
10.49 \\
460.20\end{array}$ & $\begin{array}{r}17.78 \\
361.04 \\
73.87 \\
116.34 \\
215.93 \\
12.30 \\
6.04 \\
520.60 \\
31.32 \\
1355.22\end{array}$ \\
\hline BAYFIELO & $\begin{array}{l}\text { WASHBU,RN CITY OF } \\
\text { RAYFIFLD CITY OF } \\
\text { TOTAL }\end{array}$ & $\begin{array}{l}\text { GW } \\
\text { GW }\end{array}$ & $\begin{array}{r}1972 \\
883 \\
2855\end{array}$ & $\begin{array}{l}25.47 \\
13.50 \\
38.97\end{array}$ & $\begin{array}{l}5.93 \\
0.0 \\
5.93\end{array}$ & $\begin{array}{r}8.09 \\
12.40 \\
20.49\end{array}$ & $\begin{array}{l}\begin{array}{l}1.60 \\
30.55 \\
72.15\end{array}\end{array}$ & $\begin{array}{r}81.09 \\
56.45 \\
137.54\end{array}$ \\
\hline BROWN & $\begin{array}{l}\text { ALLOUF Z TOWN OF } \\
\text { ASHWAUBENON VILLAGE OF } \\
\text { DENMARK VILLAGE OF } \\
\text { DE PERE CITY OF } \\
\text { GREEN PAY CITY OF } \\
\text { HDWARO VILLAGE OF } \\
\text { PULASKI VILLAGE OF } \\
\text { WRIGHTSTOWN VILLAGE OF } \\
\text { TOTAL. }\end{array}$ & $\begin{array}{l}\text { GW } \\
G W \\
G W \\
G W \\
S W \\
G W \\
G W\end{array}$ & $\begin{array}{r}14638 \\
15315 \\
1665 \\
14913 \\
89918 \\
7842 \\
1874 \\
1192 \\
147357\end{array}$ & $\begin{array}{r}315.76 \\
262.57 \\
20.24 \\
326.38 \\
1570.00 \\
111.62 \\
30.00 \\
16.05 \\
2653.21\end{array}$ & $\begin{array}{r}0.0 \\
117.61 \\
6.67 \\
73.52 \\
2330.00 \\
1.21 \\
12.70 \\
1.13 \\
2542.84\end{array}$ & $\begin{array}{r}36.97 \\
206.60 \\
7.88 \\
43.48 \\
927.00 \\
32.28 \\
10.40 \\
3.17 \\
1267.79\end{array}$ & $\begin{array}{r}203.06 \\
184.17 \\
23.91 \\
96.23 \\
958.00 \\
54.42 \\
6.52 \\
7.83 \\
1534.13\end{array}$ & $\begin{array}{r}555.79 \\
770.95 \\
58.70 \\
539.60 \\
5785.00 \\
199.53 \\
59.62 \\
28.78 \\
1997.91\end{array}$ \\
\hline BUFFALO & $\begin{array}{l}\text { ALMA CITY OF } \\
\text { COCHRANE VILLAGE OF } \\
\text { FOUNTAIN CITY CITY OF } \\
\text { MONOOOVI CITY OF } \\
\text { NELSON VILLAGE OF } \\
\text { TOTAL. }\end{array}$ & $\begin{array}{l}G W \\
G W \\
G W \\
G W \\
G W\end{array}$ & $\begin{array}{r}942 \\
603 \\
1093 \\
2391 \\
383 \\
5412\end{array}$ & $\begin{array}{r}25.60 \\
7.17 \\
2.72 \\
37.20 \\
4.77 \\
77.46\end{array}$ & $\begin{array}{l}0.0 \\
0.33 \\
0.00 \\
10.20 \\
0.43 \\
10.95\end{array}$ & $\begin{array}{r}13.30 \\
3.77 \\
2.93 \\
22.70 \\
3.75 \\
46.45\end{array}$ & $\begin{array}{r}11.98 \\
9.89 \\
0.25 \\
21.80 \\
1.94 \\
45.86\end{array}$ & $\begin{array}{r}50.88 \\
21.16 \\
51.90 \\
91.90 \\
10.88 \\
180.73\end{array}$ \\
\hline BURNETT & $\begin{array}{l}\text { GRANTSBURG VILLLAGE OF } \\
\text { SIREN VILLAGE OF } \\
\text { WEESTER VILLAGE OF } \\
\text { TOTAL. }\end{array}$ & $\begin{array}{l}\text { GW } \\
\text { GW } \\
\text { GW }\end{array}$ & $\begin{array}{r}1102 \\
841 \\
580 \\
2523\end{array}$ & $\begin{array}{l}11.83 \\
12.46 \\
8.89 \\
33.18\end{array}$ & $\begin{array}{l}10.63 \\
0.0 \\
0.0 \\
10.63\end{array}$ & $\begin{array}{l}15.59 \\
15.37 \\
5.18 \\
36.15\end{array}$ & $\begin{array}{r}9.91 \\
6.31 \\
11.07 \\
27.29\end{array}$ & $\begin{array}{r}47.96 \\
34.14 \\
25.14 \\
107.24\end{array}$ \\
\hline CALUMET & $\begin{array}{l}\text { ARILLION CITY OF } \\
\text { CHILTON CITY OF } \\
\text { HILAERT VILLAGE OF } \\
\text { NEW HOLSTEIN CITY OF } \\
\text { SHERWNOD VILLAGE OF } \\
\text { TOTAL. }\end{array}$ & $\begin{array}{l}G W \\
G W \\
G W \\
G W \\
G W\end{array}$ & $\begin{array}{r}2752 \\
2929 \\
1075 \\
3356 \\
395 \\
10507\end{array}$ & $\begin{array}{r}50.03 \\
45.54 \\
15.61 \\
57.10 \\
5.60 \\
173.90\end{array}$ & $\begin{array}{r}228.71 \\
191.36 \\
4.89 \\
74.48 \\
0.0 \\
499.45\end{array}$ & $\begin{array}{l}17.15 \\
24.35 \\
4.54 \\
16.32 \\
1.98 \\
64.33\end{array}$ & $\begin{array}{r}4.98 \\
14.02 \\
5.45 \\
19.19 \\
11.46 \\
55.10\end{array}$ & $\begin{array}{l}300.87 \\
275.28 \\
30.49 \\
167.10 \\
19.04 \\
792.78\end{array}$ \\
\hline CHIPPFWA & $\begin{array}{l}\text { ALOOMFR CITY OF } \\
\text { AOYD VILLAGE OF } \\
\text { CADOTT VILLAGE OF } \\
\text { CHIPPFWA FALLS CITY OF } \\
\text { CORNELL CITY OF } \\
\text { NEW AUBURN VILLAGE OF } \\
\text { STANLFY CITY OF } \\
\text { TOTAL }\end{array}$ & $\begin{array}{l}G W \\
G W \\
G W \\
G W \\
G W \\
G W \\
G W\end{array}$ & $\begin{array}{r}3371 \\
622 \\
1242 \\
12658 \\
1535 \\
415 \\
2004 \\
21847\end{array}$ & $\begin{array}{r}56.11 \\
7.37 \\
18.40 \\
189.54 \\
17.00 \\
6.71 \\
27.66 \\
322.80\end{array}$ & $\begin{array}{r}103.23 \\
0.69 \\
3.45 \\
673.99 \\
5.27 \\
0.0 \\
9.83 \\
796.45\end{array}$ & $\begin{array}{r}24.24 \\
2.31 \\
5.83 \\
89.95 \\
8.43 \\
2.08 \\
12.86 \\
145.71\end{array}$ & $\begin{array}{r}10.08 \\
6.46 \\
9.55 \\
221.19 \\
13.06 \\
5.51 \\
50.67 \\
316.52\end{array}$ & $\begin{array}{r}193.67 \\
16.83 \\
37.23 \\
1174.67 \\
43.76 \\
14.30 \\
101.02 \\
1581.48\end{array}$ \\
\hline CLARK & $\begin{array}{l}\text { ABBOTSFORO CITY OF } \\
\text { COLAY CITY OF } \\
\text { OORCESTER VILLAGE OF } \\
\text { GRANTON VILLAGE OF } \\
\text { GREENWOOO CIIY OF } \\
\text { LOYAL CITY OF } \\
\text { NEILLSVILLE CITY OF } \\
\text { OWEN CITY OF } \\
\text { THORP CITY OF } \\
\text { WITHEF VILLAGE OF } \\
\text { TOTAL. }\end{array}$ & $\begin{array}{l}\text { GW } \\
G W \\
G W \\
G W \\
G W \\
G W \\
G W \\
G W \\
G W \\
G W\end{array}$ & $\begin{array}{r}1895 \\
1353 \\
598 \\
347 \\
1176 \\
1252 \\
2881 \\
958 \\
1683 \\
491 \\
12634\end{array}$ & $\begin{array}{r}24.87 \\
16.40 \\
7.28 \\
5.15 \\
15.65 \\
17.80 \\
35.30 \\
10.90 \\
34.80 \\
6.43 \\
174.58\end{array}$ & $\begin{array}{r}14.63 \\
6.28 \\
3.43 \\
0.0 \\
18.55 \\
3.12 \\
43.10 \\
3.79 \\
1.04 \\
0.06 \\
94.00\end{array}$ & $\begin{array}{r}16.44 \\
9.40 \\
2.50 \\
0.600 \\
2.82 \\
6.80 \\
27.90 \\
7.98 \\
14.50 \\
3.05 \\
92.00\end{array}$ & $\begin{array}{r}18.22 \\
7.41 \\
4.21 \\
3.11 \\
13.96 \\
2.79 \\
26.05 \\
14.26 \\
17.36 \\
9.30 \\
116.68\end{array}$ & $\begin{array}{r}74.16 \\
39.49 \\
17.42 \\
8.86 \\
50.97 \\
30.51 \\
132.35 \\
36.93 \\
67.70 \\
18.85 \\
477.25\end{array}$ \\
\hline COLUMBIA & $\begin{array}{l}\text { ARLINGTUN VILLAGE OF } \\
\text { CAMBRIA VILLAGE OF } \\
\text { COLUMAUS CIIY OF } \\
\text { FALL RIVER VILLAGE OF } \\
\text { FRIESLAND VILLAG OF } \\
\text { LOIDI CITY OF } \\
\text { PARDEFVILLE VILLAGE OF } \\
\text { PDRTAGE CIYY OF } \\
\text { POYNETIE VILLAGE OF } \\
\text { RANDOLP VILLAGE OF } \\
\text { RIO VILLAGE OF } \\
\text { WISCONSIN OELLS CITY OF } \\
\text { WYOCENA VILLAGE OF } \\
\text { TDTAL }\end{array}$ & $\begin{array}{l}G w \\
G W \\
G W \\
G W \\
G W \\
G W \\
G W \\
G W \\
G W \\
G W \\
G W \\
G W \\
G W\end{array}$ & $\begin{array}{r}406 \\
683 \\
3966 \\
791 \\
289 \\
2123 \\
1695 \\
7655 \\
1497 \\
1306 \\
859 \\
2447 \\
447 \\
24164\end{array}$ & $\begin{array}{r}8.63 \\
12.64 \\
66.40 \\
12.20 \\
4.09 \\
35.50 \\
28.60 \\
178.00 \\
23.44 \\
25.22 \\
18.53 \\
43.80 \\
4.54 \\
461.59\end{array}$ & $\begin{array}{l}0.11 \\
20.12 \\
19.80 \\
24.50 \\
1.00 \\
37.40 \\
0.0 \\
80.00 \\
2.45 \\
6.32 \\
2.15 \\
0.0 \\
0.0 \\
194.04\end{array}$ & $\begin{array}{r}1.72 \\
4.52 \\
34.00 \\
7.86 \\
0.14 \\
20.70 \\
9.13 \\
89.90 \\
11.52 \\
6.26 \\
1.54 \\
77.20 \\
0.99 \\
265.79\end{array}$ & $\begin{array}{r}5.38 \\
13.25 \\
41.22 \\
13.46 \\
1.22 \\
29.20 \\
16.81 \\
88.80 \\
14.60 \\
11.11 \\
7.83 \\
49.29 \\
11.96 \\
304.14\end{array}$ & $\begin{array}{r}15.84 \\
50.54 \\
161.42 \\
58.02 \\
6.45 \\
122.80 \\
54.54 \\
437.30 \\
52.02 \\
48.91 \\
30.05 \\
170.59 \\
17.49 \\
1225.96\end{array}$ \\
\hline CRAWFORD & $\begin{array}{l}\text { EASTMAN VILLAGE OF } \\
\text { GAYS MILLS VILLAGE DF } \\
\text { MOUNT STERLING VILLAGE O } \\
\text { PQAIRIE DU CHIEN CITY OF } \\
\text { SOLDIEHS GROVE VILLAGE O } \\
\text { WAUZEKA VILLAGE OF } \\
\text { TOTAL. }\end{array}$ & $\begin{array}{l}\text { GW } \\
G W \\
G W \\
G W \\
G W \\
G W\end{array}$ & $\begin{array}{r}330 \\
661 \\
157 \\
5544 \\
507 \\
456 \\
7655\end{array}$ & $\begin{array}{r}2.79 \\
8.18 \\
2.91 \\
108.00 \\
5.64 \\
7.44 \\
134.96\end{array}$ & $\begin{array}{c}0.0 \\
4.37 \\
1.14 \\
65.20 \\
1.48 \\
0.0 \\
72.19\end{array}$ & $\begin{array}{r}3.40 \\
2.20 \\
0.26 \\
62.10 \\
3.68 \\
1.03 \\
72.67\end{array}$ & $\begin{array}{r}1.60 \\
15.21 \\
0.49 \\
213.50 \\
18.03 \\
11.75 \\
260.58\end{array}$ & $\begin{array}{r}7.79 \\
29.96 \\
4.80 \\
448.80 \\
28.83 \\
20.22 \\
540.40\end{array}$ \\
\hline
\end{tabular}


Table 1.--Municipal water use in 1979--Continued

WATER DELIVERIES IN MILLIONS OF GALLONS

counTr

MUNICIPALITY
SOURCE POPULATION

DANE

RFLLEVILLE VILLAGE OF AL ACKEARTH VILLAGE OF CAMBRIDGE VILLAGE OF COTTAGE GROVE VILLAGE OF
CROSS PLAINS VILLAGE DF CROSS PLAINS VILLA DANE VILLAGE DF
DEERFIELO VILLAGE OF DEERFIELN VILLAGE OF FITCHRURG TOWN OF MADISON CITY OF MAPLE BLUFF VILLAGE OF MARSHALL VILLAGE OF MAZOMANIE VILLAGE OF MC FARLAND VILLAGE OF MIDDLF TON CITY OF

MONONA CITY OF MOUNT HOREH VILLAGE SHEREWOON HLS VILLAGE OF SHOREWOON HLS VILLAI SUN PRAIRIE CITY OF SUN PRAIRIE CITY OF

VAUNAKEE VILLAGE OF TOTAL

DODGE

REAVED DAM CITY OF RROWNGVILLE VILLAGE OF CLYMAN VILLAGE OF FDX LAKE CITY OF HDRICON CITY OF HUSTISFORD VILLAGE OF IRON RIDGE VILLA JUNEAU CITY OF LOMIRA VILLAGE OF LOWELL VILLAGE OF RFESEVILLE VILLAGE OF THERESA VILLAGE OF WAUPUN CITY OF

DOOR

SISTER BAY VILLAGE OF STURGFON BAY CITY OF

DOUGLAS

oliver VILLAGE of SUPERTOR CITY OF TOTAL

DUNN

AOYCEVILLE VILLAGE OF CDLFAX VILLAGE OF ELK MNUN VILLAGE OF KNAPP VILLAGE OF MENOMONIE CIIY OF WHEELFR

EAU CLAIRE

aL TOONA CITY OF EAU CLAIRE CITY OF FAIRCHILO VILLAGE OF FALL CREEK VILLAGE OF TOTAL

FOND DU LAC

gRANDON VILLAGE OF CAMPBELLSPORT VILLAGE OF FAIRWATER VILLAGE OF NOR FOND DU LAC VILLG OF NARFIFLD VILLAGE OF DAKFIFLD VILLAGE OF ST CLNUD VILLAGE TOTAL

FOREST

CRANDON CITY OF TOTAL

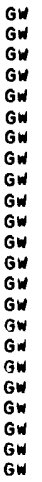

RAGLEY VILLAGE OF

BLOOMINGTON VILLAGE OF ALUE RIVER VILLAGE OF RO5COREL CITY OF

CASSVTLLE VILLAGE OF

DICKEYVILLE VILLAGE OF FEN IMORF CITY OF HAZEL GREEN VILLAGE OF HAZEL GREEN VILLAGE LIVINFSTON VILLAGE OF MONTFORT VILLAGE OF MOUNT HOPE VILLAGE OF MOUT HOPE VILLAGE OF PATCH GROVE VILLAGE OF PLATTFVILLE CIIY OF PLATTFVILLE CITY OF TENAYCON VILLAGE OF TENAL

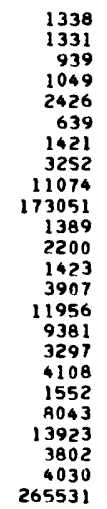

RESIDENTIAL

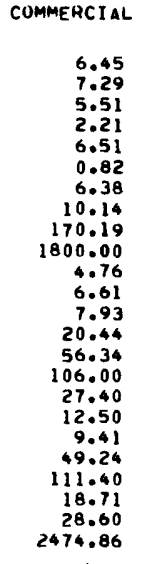

104.17

3.63
1.38

249.00

7.60
4.12

4.12
24.60

67.36

12.00

7.33
28.16

22.84

4.03
70.37

70.37
7.94

7.94
10.87

109.00
625.23

9.39

164.00
173.39

1.46
472.00

473.46

17.40
16.46

7.88

10.10
144.00

7.05
202.90

70.79

18.43
1010.00

010.00
5.66

16.56

1121.44

14.00
24.40
4.63

4.63
704.00

69.88
19.60

19.60
8.52

121.30
966.33

966.33

22.70
22.70

5.11

12.00
6.80

6.80
12.70
19.60

19.60
36.40

36.40
20.20

32.9

20.40
70.10

11.42

9.98
4.00

19.40

4.36

134.00

10.15
9.54
227.29

0.0

49.87
0.97

97.67

14.23

0.52
23.90

6.50

0.13
134.20

1.45

1.97

35.40
594.10

0.0
213.00

213.00
213.00

0.0
588.00

588.00
588.00

3.00
0.18

0.0

0.31
27.10

0.0
30.59

0.0
116.15
1500.0

1500.00
0.0

0.0

0.0
1616.15

0.0
20.40

1.13

475.00

9.61
31.80

0.0
104.90

642.83

3.97
3.97

$$
\begin{aligned}
& 0.0 \\
& 0.0 \\
& 0.0
\end{aligned}
$$

0.0

26.80

26.80
4.78

1.52
13.30

13.30
0.0

93.90
4.16

4.16
1.11

0.0

0.0
0.0

23.45

0.0
0.0
169.02
13.21

4.25
6.87

6.88
8.32

0.48
27.68

5.47

2.27
38.70

38.70
225.92

8.71
103.00

111.71

0.35
323.00

323.35

3.96
6.42

6.42
4.79

2.34
199.00

1.63
218.15

37.23
8.44
456.00
2.70
4.66

2.45

18.70
0.84
211.00

211.00
16.23

2.73

0.45
91.86

91.86
34.27

18.60
18.60

0.95

6.14

50.80

7.33
12.30

2.00
14.00

14.00
13.40

13.40
26.50
1.75

1.75
1.45

2.00
+4.50

4.50
0.31

34.87

2.30
1.72
OTHER

TOTAL

33.93
6.70
12.46
2.60
5.19
4.91
2.64
10.76
66.09
3264.00
4.58
12.06
14.32
37.69
122.16
9.67
105.78
39.80
15.90
174.65
160.85
30.28
31.21
4168.25

151.49

10.61
3.57

3.57
25.57

38.15
6.20

2.99

49.81
7.32

2.52
42.21

3.47
5.34

5.34
169.60
518.84

3.29

187.00
190.29

0.62

291.20
291.82

5.64
17.57
7.60

7.60
2.81

2.81
126.00

0.65
160.26

37.25
17.41

17.41
856.00

4.81
10.27

10.27
925.73

11.30
31.54

1.76
1582.00

1582.00
22.17

6.64

03.20
142.24

1742.24

17.56
17.56

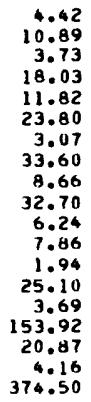

10.89

18.03

11.82
23.80

3.07
33.60

8.66

6.24

1.94
25.10

3.69
153.92

4.16
374.50

69.85

32.75
32.02

28.36

66.48

17.67

46.10

137.81
328.83

10324.00

74.84

63.03

46.61
144.32

589.21

335.67

208.68

134.73 


\section{Table 1.--Municipal water use in 1979--Continued}

\begin{tabular}{|c|c|c|c|c|c|c|c|c|}
\hline COUNTY & MUNICIPALITY & SOURCE & POPULATIDN & RESIDENTIALL & INDUSTRIAL & COMMERCIAL & OTHER & TOTAL \\
\hline GREEN & $\begin{array}{l}\text { ALBANY VILLAGE OF } \\
\text { BROOHEAO CIIYY OF } \\
\text { BROOKLYN VILLAGE OF } \\
\text { RROWNTOWN VILLAGE DF } \\
\text { MDNROE CITY OF } \\
\text { MONTICELLO VILLAGE OF } \\
\text { NEW GI.ARUS VILLAGE OF } \\
\text { TOTAL. }\end{array}$ & $\begin{array}{l}\text { GW } \\
\text { GW } \\
G W \\
G W \\
G W \\
\text { GW }\end{array}$ & $\begin{array}{r}1001 \\
3113 \\
673 \\
272 \\
9618 \\
955 \\
1628 \\
17260\end{array}$ & $\begin{array}{r}15.60 \\
58.80 \\
13.19 \\
4.33 \\
170.00 \\
17.70 \\
26.40 \\
306.02\end{array}$ & $\begin{array}{r}0.01 \\
18.90 \\
0.03 \\
0.03 \\
234.00 \\
6.93 \\
0.0 \\
259.90\end{array}$ & $\begin{array}{r}4.35 \\
11.60 \\
1.78 \\
0.90 \\
187.00 \\
8.22 \\
27.30 \\
241.14\end{array}$ & $\begin{array}{r}18.23 \\
26.93 \\
5.22 \\
0.32 \\
276.40 \\
16.57 \\
39.53 \\
383.20\end{array}$ & $\begin{array}{r}38.19 \\
116.23 \\
20.21 \\
5.57 \\
867.40 \\
49.42 \\
93.23 \\
1190.26\end{array}$ \\
\hline GREEN LAKE & $\begin{array}{l}\text { RERLIN CITY OF } \\
\text { GREEN LAKE CITY OF } \\
\text { MARKESAN CITY OF } \\
\text { PRINCFTON CITY OF } \\
\text { TOTAL }\end{array}$ & $\begin{array}{l}\text { GW } \\
\text { GW } \\
G W \\
G W\end{array}$ & $\begin{array}{l}5286 \\
1124 \\
1412 \\
1386 \\
9208\end{array}$ & $\begin{array}{r}93.90 \\
22.50 \\
23.10 \\
22.00 \\
161.50\end{array}$ & $\begin{array}{r}192.00 \\
0.0 \\
15.00 \\
2.52 \\
209.52\end{array}$ & $\begin{array}{r}50.80 \\
17.80 \\
6.34 \\
18.00 \\
92.94\end{array}$ & $\begin{array}{l}33.30 \\
10.55 \\
15.70 \\
22.40 \\
81.95\end{array}$ & $\begin{array}{r}370.00 \\
50.85 \\
60.14 \\
64.92 \\
545.91\end{array}$ \\
\hline IOWA & $\begin{array}{l}\text { ARENA VILLAGE OF } \\
\text { AVOCA VILLAGE OF } \\
\text { RARNEVELD VILLAGE OF } \\
\text { COBB VILLAGE OF } \\
\text { NOOGEVILLE ITY OF } \\
\text { HIGHLAND VILLAGE OF } \\
\text { HOLLANDALE VILLAGE OF } \\
\text { LINDEN VILLAGE OF } \\
\text { MINERAL POINT CITY OF } \\
\text { MINEY VILLAGE OF } \\
\text { RFWEY VILAGE } \\
\text { RIDGEWAY VILLAGE OF } \\
\text { TOTAL }\end{array}$ & $\begin{array}{l}G W \\
G W \\
G W \\
G W \\
G W \\
G W \\
G W \\
G W \\
G W \\
G W\end{array}$ & $\begin{array}{r}446 \\
464 \\
603 \\
418 \\
3426 \\
761 \\
293 \\
386 \\
2360 \\
235 \\
505 \\
9897\end{array}$ & $\begin{array}{r}8.01 \\
6.49 \\
9.56 \\
5.89 \\
67.10 \\
11.84 \\
3.18 \\
5.35 \\
33.51 \\
3.39 \\
7.03 \\
161.35\end{array}$ & $\begin{array}{l}4.03 \\
1.83 \\
6.07 \\
5.67 \\
13.77 \\
0.0 \\
0.00 \\
2.31 \\
8.118 \\
1.83 \\
0.00 \\
43.69\end{array}$ & $\begin{array}{l}0.65 \\
0.0 \\
0.0 \\
1.10 \\
0.0 \\
2.38 \\
11.70 \\
2.26 \\
18.97 \\
1.85 \\
1.87 \\
30.78\end{array}$ & $\begin{array}{r}2.15 \\
5.59 \\
27.66 \\
6.87 \\
114.17 \\
7.52 \\
2.92 \\
14.20 \\
93.28 \\
6.35 \\
7.78 \\
288.48\end{array}$ & $\begin{array}{r}14.85 \\
13.91 \\
43.29 \\
19.53 \\
195.03 \\
21.74 \\
7.80 \\
24.11 \\
153.94 \\
13.43 \\
16.68 \\
524.30\end{array}$ \\
\hline IRON & $\begin{array}{l}\text { HURLEY CITY OF } \\
\text { MONTAFAL CITY OF } \\
\text { TOTAL }\end{array}$ & $\begin{array}{l}G W \\
G W\end{array}$ & $\begin{array}{l}2139 \\
791 \\
2930\end{array}$ & $\begin{array}{l}32.64 \\
14.00 \\
46.64\end{array}$ & $\begin{array}{l}0.0 \\
0.0 \\
0.0\end{array}$ & $\begin{array}{l}21.01 \\
4.00 \\
25.01\end{array}$ & $\begin{array}{l}71.56 \\
17.00 \\
88.56\end{array}$ & $\begin{array}{r}125.22 \\
35.00 \\
160.22\end{array}$ \\
\hline JACKSON & $\begin{array}{l}\text { ALMA CENTER VILLAGE OF } \\
\text { RLACK RIVER FALLS CITY } \\
\text { HIXTON VILLAGE OF } \\
\text { MELROSE VILLAGE OF } \\
\text { MERRILLAN VILLAGE OF } \\
\text { TAYLOR VILLAGE OF } \\
\text { TOTAL }\end{array}$ & $\begin{array}{l}G W \\
G W \\
G W \\
G W \\
G W \\
G W\end{array}$ & $\begin{array}{r}494 \\
3366 \\
378 \\
485 \\
608 \\
364 \\
5695\end{array}$ & $\begin{array}{r}5.82 \\
46.71 \\
6.26 \\
7.78 \\
8.31 \\
4.31 \\
79.26\end{array}$ & $\begin{aligned} 1.42 \\
27.05 \\
0.00 \\
0.0 \\
0.00 \\
0.35 \\
28.02\end{aligned}$ & $\begin{array}{r}1.85 \\
40.33 \\
2.70 \\
1.73 \\
2.86 \\
1.72 \\
51.19\end{array}$ & $\begin{array}{r}6.78 \\
03.46 \\
4.68 \\
3.27 \\
5.20 \\
3.34 \\
106.73\end{array}$ & $\begin{array}{r}15.87 \\
197.55 \\
13.64 \\
12.79 \\
16.37 \\
9.79 \\
266.01\end{array}$ \\
\hline JEFFERSON & $\begin{array}{l}\text { FORT ATKINSON CITY OF } \\
\text { JEFFERSON CITY OF } \\
\text { JOHNSON CREEK VILLAAGE OF } \\
\text { LAKE MILLS CITY OF } \\
\text { PALMYYRA VILLAGE OF } \\
\text { WATERLDO VILLAGE OF } \\
\text { WATERTOWN CITY OF } \\
\text { TOTAL. }\end{array}$ & $\begin{array}{l}G W \\
G W \\
G W \\
G W \\
G W \\
G W \\
G W\end{array}$ & $\begin{array}{r}9761 \\
5850 \\
1030 \\
3954 \\
1558 \\
2235 \\
17311 \\
41699\end{array}$ & $\begin{array}{r}224.00 \\
108.70 \\
13.64 \\
76.39 \\
26.60 \\
37.80 \\
320.08 \\
807.21\end{array}$ & $\begin{array}{r}389.00 \\
171.00 \\
21.65 \\
95.33 \\
2.95 \\
65.91 \\
356.31 \\
1102.15\end{array}$ & $\begin{array}{r}95.00 \\
48.80 \\
16.10 \\
57.66 \\
7.41 \\
23.46 \\
156.56 \\
404.98\end{array}$ & $\begin{array}{l}50.60 \\
92.60 \\
5.19 \\
98.94 \\
13.67 \\
30.34 \\
254.80 \\
546.14\end{array}$ & $\begin{array}{r}758.60 \\
421.10 \\
56.58 \\
328.32 \\
50.63 \\
157.51 \\
1087.75 \\
2860.48\end{array}$ \\
\hline JUNEAU & $\begin{array}{l}\text { CAMP NOUGLAS VILLLAGE OF } \\
\text { ELROY CITY OF } \\
\text { LYNDON STIIION VILLLGE } \\
\text { MAUSTON CITY OF } \\
\text { NECEDAH VILLAGE OF } \\
\text { NEW LISBON CITY OF } \\
\text { UNIDN CENER VILLAGE OF } \\
\text { WONEWO VILLAGE OF } \\
\text { TOTAL }\end{array}$ & $\begin{array}{l}\text { GW } \\
G W \\
G W \\
G W \\
G W \\
G W \\
G W\end{array}$ & $\begin{array}{r}592 \\
1402 \\
512 \\
3189 \\
801 \\
1343 \\
173 \\
786 \\
8798\end{array}$ & $\begin{array}{r}8.31 \\
22.85 \\
4.56 \\
62.07 \\
9.46 \\
17.20 \\
3.48 \\
12.20 \\
140.14\end{array}$ & $\begin{array}{l}0.0 \\
2.06 \\
0.15 \\
8.88 \\
6.92 \\
0.0 \\
0.0 \\
11.30 \\
29.31\end{array}$ & $\begin{array}{r}3.01 \\
7.30 \\
3.70 \\
56.33 \\
16.23 \\
17.30 \\
1.02 \\
5.06 \\
110.54\end{array}$ & $\begin{array}{l}5.19 \\
43.99 \\
15.82 \\
58.77 \\
20.73 \\
15.34 \\
1.01 \\
16.09 \\
176.94\end{array}$ & $\begin{array}{r}16.51 \\
76.21 \\
24.23 \\
186.04 \\
53.34 \\
49.84 \\
6.11 \\
44.65 \\
456.93\end{array}$ \\
\hline KENOSHA & $\begin{array}{l}\text { KENOSHA CITY OF } \\
\text { PADDOCK LAKE VILLAGE OF } \\
\text { TOTAL }\end{array}$ & $\begin{array}{l}\text { SW } \\
\text { GW }\end{array}$ & $\begin{array}{r}80775 \\
468 \\
81243\end{array}$ & $\begin{array}{l}2007.05 \\
7.41 \\
2014.46\end{array}$ & $\begin{array}{r}2060.99 \\
3.29 \\
2064.28\end{array}$ & $\begin{array}{r}558.53 \\
2.47 \\
561.00\end{array}$ & $\begin{array}{r}1855.47 \\
4.14 \\
1859.61\end{array}$ & $\begin{array}{r}6482.04 \\
17.32 \\
0499.35\end{array}$ \\
\hline KEWAIINEE & $\begin{array}{l}\text { ALGOMA CITY OF } \\
\text { KEWAUNEE CITY OF } \\
\text { LUXEMAURG VILLAGE OF } \\
\text { TOTAL }\end{array}$ & $\begin{array}{l}\text { GW } \\
\text { GW } \\
\text { GW }\end{array}$ & $\begin{array}{l}3796 \\
2898 \\
1119 \\
7813\end{array}$ & $\begin{array}{r}54.64 \\
45.33 \\
16.87 \\
116.84\end{array}$ & $\begin{array}{l}24.43 \\
20.41 \\
10.90 \\
55.73\end{array}$ & $\begin{array}{r}26.70 \\
15.21 \\
6.37 \\
48.27\end{array}$ & $\begin{array}{l}46.49 \\
27.54 \\
14.85 \\
88.87\end{array}$ & $\begin{array}{l}152.25 \\
108.49 \\
48.98 \\
309.72\end{array}$ \\
\hline LA CROSSE & $\begin{array}{l}\text { BANGOR VILLAGF OF } \\
\text { HOLMEN VILLAGE OF } \\
\text { LA CROSSE CITY OF } \\
\text { ONALASKA CITY OF } \\
\text { ROCKLAND VILLAGE OF } \\
\text { WEST SALEM VILLLGE OF } \\
\text { TOTAL }\end{array}$ & $\begin{array}{l}\text { GW } \\
G W \\
G W \\
G W \\
G W \\
G W\end{array}$ & $\begin{array}{r}1052 \\
2522 \\
49403 \\
8061 \\
350 \\
3489 \\
64877\end{array}$ & $\begin{array}{r}20.60 \\
48.20 \\
925.00 \\
183.00 \\
5.60 \\
50.60 \\
1233.00\end{array}$ & $\begin{array}{r}0.0 \\
7.76 \\
1710.00 \\
2.03 \\
0.00 \\
6.24 \\
1726.63\end{array}$ & $\begin{array}{r}12.30 \\
9.58 \\
1220.00 \\
95.90 \\
0.066 \\
36.50 \\
1374.94\end{array}$ & $\begin{array}{r}37.37 \\
44.50 \\
2611.00 \\
54.00 \\
2.88 \\
24.93 \\
2774.68\end{array}$ & $\begin{array}{r}70.27 \\
110.04 \\
6466.00 \\
335.53 \\
9.14 \\
118.27 \\
7109.24\end{array}$ \\
\hline LAFAYETTE & $\begin{array}{l}\text { ARGYLF VILLAGE OF } \\
\text { BELMONT VILLAGE OF } \\
\text { RFNION VILLAGE OF } \\
\text { BLANCHAROVILLE VILLAG OF } \\
\text { DARLINGTON CITY OF } \\
\text { GRATINT VILLAGE OF } \\
\text { SHULLSBERG CITY OF } \\
\text { SOUTH WAYNE VILLAGE OF } \\
\text { TOTAL }\end{array}$ & $\begin{array}{l}G W \\
G W \\
G W \\
G W \\
G W \\
G W \\
G W \\
G W\end{array}$ & $\begin{array}{r}532 \\
840 \\
960 \\
773 \\
2420 \\
295 \\
1450 \\
524 \\
7794\end{array}$ & $\begin{array}{r}11.70 \\
12.80 \\
14.60 \\
11.80 \\
39.80 \\
3.97 \\
25.10 \\
6.80 \\
126.57\end{array}$ & $\begin{array}{l}0.0 \\
8.36 \\
0.0 \\
11.80 \\
6.43 \\
0.0 \\
3.89 \\
0.0 \\
30.48\end{array}$ & $\begin{aligned} 8.39 \\
1.82 \\
2.31 \\
5.48 \\
14.00 \\
1.96 \\
4.70 \\
2.98 \\
41.64\end{aligned}$ & $\begin{array}{l}17.93 \\
24.03 \\
66.06 \\
31.10 \\
96.00 \\
100.94 \\
41.99 \\
18.27 \\
246.92\end{array}$ & $\begin{array}{r}38.02 \\
47.01 \\
23.57 \\
60.18 \\
156.23 \\
16.87 \\
75.68 \\
28.05 \\
445.61\end{array}$ \\
\hline AOE & $\begin{array}{l}\text { ANTIGN CITY OF } \\
\text { WHITE LAKE VILLAGE OF } \\
\text { INTAL }\end{array}$ & $\begin{array}{l}\text { GW } \\
\text { GW }\end{array}$ & $\begin{array}{r}8565 \\
308 \\
9873\end{array}$ & $\begin{array}{l}138.00 \\
4.95 \\
142.95\end{array}$ & $\begin{array}{l}101.00 \\
0.04 \\
101.04\end{array}$ & $\begin{array}{l}58.50 \\
0.80 \\
59.30\end{array}$ & $\begin{array}{l}154.80 \\
4.70 \\
159.50\end{array}$ & $\begin{array}{r}452.30 \\
10.49 \\
462.79\end{array}$ \\
\hline
\end{tabular}




\section{Table 1.--Municipal water use in 1979--Continued}

\begin{tabular}{|c|c|c|c|c|c|c|c|c|}
\hline COINNTY & MUNICIPALITY & SOURCE & POPULATION & RESIDENTIAL & \multicolumn{3}{|c|}{ WATER DELIVERIES IN MILLIONS OF GALLONS } & TOTAL \\
\hline LINCOLN & $\begin{array}{l}\text { MERRILL CITY OF } \\
\text { TOMAHAWK CITY OF } \\
\text { TOTAL }\end{array}$ & $\begin{array}{l}\text { GW } \\
\text { GW }\end{array}$ & $\begin{array}{r}9390 \\
3767 \\
13157\end{array}$ & $\begin{array}{r}148.00 \\
51.20 \\
199.20\end{array}$ & $\begin{array}{r}145.00 \\
11.60 \\
156.60\end{array}$ & $\begin{array}{r}63.40 \\
38.50 \\
101.90\end{array}$ & $\begin{array}{r}89.30 \\
59.29 \\
148.59\end{array}$ & $\begin{array}{l}445.70 \\
160.59 \\
606.29\end{array}$ \\
\hline MANITONOC & $\begin{array}{l}\text { CLEVELAND VILLAGE OF } \\
\text { KELLNERSVLLE VILLAGE OF } \\
\text { KIEL CITY OF } \\
\text { MANITOWDC CITY OF } \\
\text { MARIBFL VILLAGE OF } \\
\text { MISHICOT VILLAGE OF } \\
\text { REEDSVILLE VILLAGE OF } \\
\text { ST NAZIANZ VILLAGE OF } \\
\text { TWO RIVRS CITY OF } \\
\text { VALDERS VILLAGE OF } \\
\text { WHITELAW VILLAGE OF } \\
\text { TOTAL }\end{array}$ & $\begin{array}{l}G W \\
G W \\
G W \\
S W \\
G W \\
G W \\
G W \\
G W \\
S W \\
G W \\
G W\end{array}$ & $\begin{array}{r}1174 \\
318 \\
2715 \\
32940 \\
391 \\
1473 \\
1119 \\
787 \\
13208 \\
1054 \\
688 \\
55867\end{array}$ & $\begin{array}{r}23.30 \\
3.43 \\
53.70 \\
669.00 \\
5.14 \\
17.30 \\
19.00 \\
11.30 \\
271.00 \\
17.50 \\
10.30 \\
1100.97\end{array}$ & $\begin{array}{r}0.33 \\
0.0 \\
6.03 \\
1320.00 \\
0.10 \\
13.00 \\
0.00 \\
0.00 \\
227.00 \\
0.00 \\
0.00 \\
1567.07\end{array}$ & $\begin{array}{r}4.21 \\
3.15 \\
11.90 \\
228.00 \\
1.66 \\
0.02 \\
3.84 \\
3.64 \\
62.20 \\
12.90 \\
1.91 \\
333.43\end{array}$ & $\begin{array}{r}8.19 \\
4.03 \\
24.035 \\
271.30 \\
1.09 \\
10.76 \\
8.43 \\
6.81 \\
161.90 \\
9.52 \\
3.06 \\
510.04\end{array}$ & $\begin{array}{r}36.03 \\
10.61 \\
95.98 \\
2488.30 \\
8.59 \\
11.68 \\
31.27 \\
21.75 \\
722.10 \\
39.92 \\
15.27 \\
3511.50\end{array}$ \\
\hline MARATHON & $\begin{array}{l}\text { ATHENE VILLAGE OF } \\
\text { BRDKAW VILLAGE OF } \\
\text { EDGA VIILAGE OF } \\
\text { MARATHUN VILLAGE OF } \\
\text { MOSINFE CITY OF } \\
\text { ROTHSCHID VILLAGE OF } \\
\text { SCHOFTELI CITY OF } \\
\text { SPENCFR VILLAGE OF } \\
\text { STRRTFORO VILLAGE OF } \\
\text { WAUSAU CITY OF } \\
\text { TOTAL }\end{array}$ & $\begin{array}{l}G W \\
G W \\
G W \\
G W \\
G W \\
G W \\
G W \\
G W \\
G W \\
G W\end{array}$ & $\begin{array}{r}904 \\
251 \\
1184 \\
1558 \\
3415 \\
3635 \\
3763 \\
1720 \\
1400 \\
33342 \\
51172\end{array}$ & $\begin{array}{r}11.10 \\
5.96 \\
14.90 \\
24.20 \\
45.70 \\
65.90 \\
39.50 \\
20.40 \\
18.00 \\
608.00 \\
853.66\end{array}$ & $\begin{array}{r}0.00 \\
1900.00 \\
22.80 \\
37.00 \\
33.60 \\
94.10 \\
81.60 \\
1.79 \\
0.15 \\
329.00 \\
2500.04\end{array}$ & $\begin{array}{r}5.29 \\
0.02 \\
6.15 \\
7.74 \\
12.50 \\
14.90 \\
35.30 \\
10.70 \\
4.04 \\
378.00 \\
474.64\end{array}$ & $\begin{array}{r}12.62 \\
4.97 \\
34.20 \\
14.01 \\
33.86 \\
31.07 \\
46.710 \\
8.13 \\
11.30 \\
462.00 \\
658.86\end{array}$ & $\begin{array}{r}29.01 \\
1910.95 \\
78.05 \\
82.95 \\
125.66 \\
205.97 \\
203.10 \\
41.02 \\
33.49 \\
1777.00 \\
4487.19\end{array}$ \\
\hline MAR INETTF & $\begin{array}{l}\text { COLEMAN VILLAGE OF } \\
\text { MARINFTTE CITY OF } \\
\text { NIAGARA VILLAGE OF } \\
\text { PESHTIGO CITY OF } \\
\text { POUND VILLAGE OF } \\
\text { WAUSAIISEE VILLAGE OF } \\
\text { TOTAL }\end{array}$ & $\begin{array}{l}G W \\
S W \\
G W \\
G W \\
G W \\
G W\end{array}$ & $\begin{array}{r}789 \\
11913 \\
2305 \\
2909 \\
389 \\
590 \\
18895\end{array}$ & $\begin{array}{r}12.10 \\
216.00 \\
37.20 \\
46.10 \\
4.79 \\
10.80 \\
326.99\end{array}$ & $\begin{array}{r}28.90 \\
585.00 \\
14.10 \\
10.80 \\
0.0 \\
1.23 \\
640.03\end{array}$ & $\begin{array}{r}8.13 \\
83.10 \\
14.70 \\
13.80 \\
3.28 \\
3.96 \\
126.97\end{array}$ & $\begin{array}{r}10.60 \\
94.20 \\
106.40 \\
84.00 \\
3.85 \\
9.07 \\
308.12\end{array}$ & $\begin{array}{r}59.73 \\
978.30 \\
172.40 \\
154.70 \\
11.92 \\
25.06 \\
1402.11\end{array}$ \\
\hline MARQUETTE & $\begin{array}{l}\text { MONTELLO CITY OF } \\
\text { TOTAL }\end{array}$ & GW & $\begin{array}{l}1234 \\
1234\end{array}$ & $\begin{array}{l}25.40 \\
25.40\end{array}$ & $\begin{array}{l}0.75 \\
0.75\end{array}$ & $\begin{array}{l}13.80 \\
13.80\end{array}$ & $\begin{array}{l}8.67 \\
8.67\end{array}$ & $\begin{array}{l}48.62 \\
48.62\end{array}$ \\
\hline MILWAUKEE & 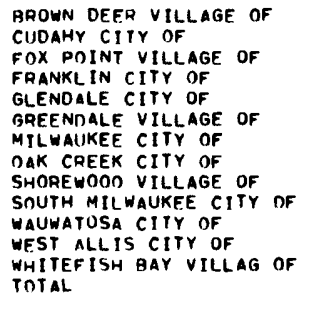 & $\begin{array}{l}\text { SW } \\
\text { SW } \\
\text { SW } \\
\text { GW } \\
\text { SW } \\
\text { SW } \\
\text { SW } \\
\text { SW } \\
\text { SW } \\
\text { SW } \\
\text { SW } \\
\text { SW } \\
\text { Sw }\end{array}$ & $\begin{array}{l}14128 \\
207 A 4 \\
7626 \\
17650 \\
13678 \\
18141 \\
612074 \\
15376 \\
13643 \\
22587 \\
53813 \\
65628 \\
15413 \\
890541\end{array}$ & $\begin{array}{r}260.00 \\
448.00 \\
291.00 \\
75.00 \\
408.00 \\
425.000 \\
14670.00 \\
207.00 \\
415.00 \\
433.00 \\
1260.00 \\
1500.00 \\
510.00 \\
20902.59\end{array}$ & $\begin{array}{r}207.00 \\
289.00 \\
0.0 \\
44.80 \\
598.00 \\
16.70 \\
18110.00 \\
608.00 \\
0.00 \\
732.00 \\
544.00 \\
1380.00 \\
0.0 \\
22529.50\end{array}$ & $\begin{array}{r}114.00 \\
123.00 \\
56.20 \\
22.40 \\
248.00 \\
108.00 \\
9080.00 \\
206.00 \\
56.30 \\
105.00 \\
442.00 \\
442.00 \\
49.40 \\
11052.29\end{array}$ & $\begin{array}{r}45.70 \\
267.60 \\
32.96 \\
\$ 1.70 \\
19.50 \\
30.40 \\
6230.00 \\
165.00 \\
94.30 \\
162.70 \\
366.30 \\
400.20 \\
115.89 \\
7972.24\end{array}$ & $\begin{array}{r}626.70 \\
1127.60 \\
380.16 \\
184.50 \\
1273.50 \\
580.10 \\
48090.001 \\
1186.00 \\
565.60 \\
1432.70 \\
2612.30 \\
3722.20 \\
675.29 \\
62456.64\end{array}$ \\
\hline MONROE & $\begin{array}{l}\text { CASHTON VILLAGE OF } \\
\text { KFNDALL VILLAGE OF } \\
\text { NORWALK VILLAGE OF } \\
\text { SPARTA CITY OF } \\
\text { TOMAH CITY OF } \\
\text { WARRENS VILLAGE OF } \\
\text { WTLTON VILLAGE OF } \\
\text { TOTAL }\end{array}$ & $\begin{array}{l}G W \\
G W \\
G W \\
G W \\
G W \\
G W \\
G W\end{array}$ & $\begin{array}{r}827 \\
444 \\
488 \\
6711 \\
7090 \\
305 \\
474 \\
16339\end{array}$ & $\begin{array}{r}19.80 \\
7.33 \\
9.03 \\
119.00 \\
103.00 \\
4.06 \\
6.92 \\
269.14\end{array}$ & $\begin{aligned} 0.0 \\
0.0 \\
11.030 \\
267.00 \\
52.30 \\
0.0 \\
0.0 \\
330.60\end{aligned}$ & $\begin{array}{r}4.79 \\
3.81 \\
2.26 \\
55.50 \\
80.70 \\
0.73 \\
2.89 \\
150.68\end{array}$ & $\begin{array}{r}16.92 \\
1.84 \\
16.12 \\
247.80 \\
26.40 \\
0.54 \\
16.30 \\
325.92\end{array}$ & $\begin{array}{r}41.51 \\
12.98 \\
38.71 \\
689.30 \\
262.40 \\
5.33 \\
26.11 \\
1076.34\end{array}$ \\
\hline OCONTO & $\begin{array}{l}\text { GILLETT CITY OF } \\
\text { LENA VILLAGE OF } \\
\text { OCONTO CITY OF } \\
\text { OCONTO FALLS CITY OF } \\
\text { SURING VILLAGE OF } \\
\text { TOTAL }\end{array}$ & $\begin{array}{l}G W \\
G W \\
G W \\
G W \\
G W\end{array}$ & $\begin{array}{l}1306 \\
643 \\
4661 \\
2684 \\
573 \\
9867\end{array}$ & $\begin{array}{r}21.80 \\
9.37 \\
62.50 \\
46.90 \\
8.27 \\
148.84\end{array}$ & $\begin{array}{l}61.20 \\
59.90 \\
59.10 \\
28.60 \\
0.00 \\
209.80\end{array}$ & $\begin{array}{r}6.85 \\
4.01 \\
21.20 \\
18.90 \\
5.06 \\
56.02\end{array}$ & $\begin{array}{r}15.43 \\
27.40 \\
74.60 \\
25.16 \\
7.77 \\
150.36\end{array}$ & $\begin{array}{l}105.28 \\
100.68 \\
217.40 \\
119.56 \\
21.10 \\
564.02\end{array}$ \\
\hline DNEIUA & $\begin{array}{l}\text { RHINEL ANDER CITY OF } \\
\text { TOTAL }\end{array}$ & Gw & $\begin{array}{l}8407 \\
8407\end{array}$ & $\begin{array}{l}145.00 \\
145.00\end{array}$ & $\begin{array}{l}190.00 \\
190.00\end{array}$ & $\begin{array}{l}120.00 \\
120.00\end{array}$ & $\begin{array}{l}301.30 \\
301.30\end{array}$ & $\begin{array}{l}756.30 \\
756.30\end{array}$ \\
\hline OUTAGAMIE & $\begin{array}{l}\text { APPLETON CITY OF } \\
\text { RLACK CRFEK VILLAGE OF } \\
\text { COMEINEO LOCKS VILLGE OF } \\
\text { HORONVILLE VILLAE OF } \\
\text { KAUKAUNA CITY OF } \\
\text { KIMERLY VILLAGE OF } \\
\text { LITTLE CHUTE VILLAGE OF } \\
\text { NICOOLS VILLAGE OF } \\
\text { SEYMOUR CITY OF } \\
\text { SHIOCTON VILLAGE OF } \\
\text { TOTAL }\end{array}$ & $\begin{array}{l}\text { SW } \\
G W \\
G W \\
G W \\
G W \\
G W \\
G W \\
G W \\
G W \\
G W\end{array}$ & $\begin{array}{r}62170 \\
1131 \\
3234 \\
2022 \\
11802 \\
6285 \\
7297 \\
312 \\
2509 \\
917 \\
91679\end{array}$ & $\begin{array}{r}1110.00 \\
23.90 \\
45.60 \\
30.70 \\
210.00 \\
112.00 \\
131.00 \\
3.36 \\
42.10 \\
11.30 \\
1719.96\end{array}$ & $\begin{array}{r}974.00 \\
61.60 \\
18.70 \\
5.84 \\
86.40 \\
130.00 \\
11.40 \\
0.00 \\
91.30 \\
0.0 \\
1379.24\end{array}$ & $\begin{array}{r}395.00 \\
7.08 \\
3.16 \\
4.45 \\
71.20 \\
9.11 \\
46.10 \\
11.43 \\
11.80 \\
4.13 \\
553.46\end{array}$ & $\begin{array}{r}504.00 \\
2.34 \\
0.74 \\
26.25 \\
145.80 \\
36.70 \\
37.78 \\
3.85 \\
114.50 \\
5.14 \\
877.10\end{array}$ & $\begin{array}{r}2983.00 \mathrm{~N} \\
94.92 \\
68.20 \\
67.24 \\
513.40 \\
287.81 \mathrm{~W} \\
226.28 \\
8.64 \\
259.70 \\
20.57 \\
4529.75\end{array}$ \\
\hline OZAUKEE & $\begin{array}{l}\text { RFLGIUM VILLAGE OF } \\
\text { CEOARRURG CCIYY OF } \\
\text { FREDONIA VILLAGE OF } \\
\text { GRAFTNN VILAAE OF } \\
\text { PORT WASHILATON CITY OF } \\
\text { SAUKVILLF VILLAGE OF } \\
\text { TOTAL }\end{array}$ & $\begin{array}{l}G W \\
G W \\
G W \\
G W \\
S W \\
G W\end{array}$ & $\begin{array}{r}865 \\
10438 \\
1600 \\
8955 \\
8639 \\
3654 \\
34151\end{array}$ & $\begin{array}{r}15.20 \\
232.00 \\
26.50 \\
171.00 \\
180.00 \\
68.00 \\
692.70\end{array}$ & $\begin{array}{l}45.50 \\
115.00 \\
1.06 \\
196.00 \\
102.00 \\
214.00 \\
674.036\end{array}$ & $\begin{array}{r}6.20 \\
48.00 \\
6.44 \\
67.40 \\
45.60 \\
5.70 \\
179.34\end{array}$ & $\begin{array}{r}6.64 \\
106.40 \\
13.94 \\
90.20 \\
88.30 \\
4.10 \\
309.57\end{array}$ & $\begin{array}{r}73.54 \\
501.40 \\
48.74 \\
524.60 \\
415.90 \\
291.80 \\
1855.97\end{array}$ \\
\hline
\end{tabular}


Table 1.--Municipal water use in 1979--Continued

WATER DELIVEHIES IN MILLLIONS OF GALLONS

\begin{tabular}{|c|c|c|c|c|c|c|c|c|}
\hline COUNTY & MUNICIPALITY & SOURCE & POPULATION & RESIDENTI AL & INDUSTRIAL & COMMERCIAL & OTHER & TOTAL \\
\hline PEPIN & $\begin{array}{l}\text { DURANN CITY OF } \\
\text { PFPIN VILLAGE OF } \\
\text { TOTAL }\end{array}$ & $\begin{array}{l}\text { GW } \\
\text { GW }\end{array}$ & $\begin{array}{r}2104 \\
763 \\
2867\end{array}$ & $\begin{array}{l}29.20 \\
13.80 \\
43.00\end{array}$ & $\begin{array}{r}18.70 \\
0.35 \\
19.05\end{array}$ & $\begin{array}{r}13.90 \\
6.17 \\
20.07\end{array}$ & $\begin{array}{r}34.10 \\
\$ .58 \\
38.68\end{array}$ & $\begin{array}{r}95.90 \\
24.90 \\
120.80\end{array}$ \\
\hline PIERCE & $\begin{array}{l}\text { ELLSWORTH VILLAGE OF } \\
\text { ELMWOAD VILLAGE OF } \\
\text { PRESCAT CITY OF } \\
\text { RIVER FALLS CITY OF } \\
\text { SPRINA VALLEY VILLAGE OF } \\
\text { TOTAL }\end{array}$ & $\begin{array}{l}\text { GW } \\
\text { GW } \\
\text { GW } \\
\text { GW } \\
\text { GW }\end{array}$ & $\begin{array}{r}2056 \\
839 \\
2738 \\
8276 \\
994 \\
14903\end{array}$ & $\begin{array}{r}42.60 \\
12.60 \\
46.80 \\
141.00 \\
14.40 \\
257.40\end{array}$ & $\begin{array}{l}0.14 \\
0.0 \\
0.0 \\
0.19 \\
0.0 \\
0.33\end{array}$ & $\begin{array}{r}23.80 \\
5.70 \\
18.70 \\
54.70 \\
11.50 \\
114.40\end{array}$ & $\begin{array}{r}14.24 \\
14.11 \\
51.99 \\
126.70 \\
27.90 \\
234.94\end{array}$ & $\begin{array}{r}80.78 \\
32.41 \\
117.49 \\
322.59 \\
53.80 \\
607.07\end{array}$ \\
\hline POLK & $\begin{array}{l}\text { AMERY CITY OF } \\
\text { RALSAM LAKE VILLLAGE OF } \\
\text { CENTURIA VILLAGE OF } \\
\text { CLAYTON VILLAGE OF } \\
\text { CLEAR LAKE VILLAGE OF } \\
\text { DRESSF VILLAGE OF } \\
\text { FREDERIC VILLAGE OF } \\
\text { LUCK VILLAGE OF } \\
\text { MILLTOWN VILLAGE } \\
\text { OSCEOLA VILLAGE OF } \\
\text { ST CROIX FALLS CITY OF } \\
\text { TOTAL }\end{array}$ & $\begin{array}{l}\text { GW } \\
\text { GW } \\
\text { GW } \\
\text { GW } \\
\text { GW } \\
\text { GW } \\
\text { GW } \\
\text { GW } \\
\text { GW } \\
\text { GW } \\
\text { GW }\end{array}$ & $\begin{array}{r}2430 \\
742 \\
704 \\
378 \\
843 \\
691 \\
1024 \\
913 \\
723 \\
1498 \\
1593 \\
11539\end{array}$ & $\begin{array}{r}32.00 \\
8.41 \\
7.71 \\
7.92 \\
13.10 \\
9.82 \\
23.30 \\
14.80 \\
9.20 \\
19.20 \\
20.50 \\
165.96\end{array}$ & $\begin{array}{r}6.20 \\
0.0 \\
0.0 \\
21.40 \\
88.10 \\
49.60 \\
70.30 \\
3.97 \\
0.20 \\
21.40 \\
23.50 \\
284.67\end{array}$ & $\begin{array}{r}25.70 \\
6.69 \\
10.80 \\
1.70 \\
3.99 \\
8.09 \\
2.48 \\
8.50 \\
1.74 \\
14.60 \\
24.70 \\
114.99\end{array}$ & $\begin{array}{r}36.00 \\
12.28 \\
7.98 \\
3.02 \\
32.45 \\
16.17 \\
14.86 \\
21.01 \\
15.06 \\
27.21 \\
19.64 \\
205.68\end{array}$ & $\begin{array}{r}99.90 \\
27.38 \\
26.49 \\
34.04 \\
137.64 \\
83.68 \\
110.94 \\
48.28 \\
32.19 \\
82.41 \\
88.34 \\
771.29\end{array}$ \\
\hline PORTAGE & $\begin{array}{l}\text { AMHERST VILLAGE OF } \\
\text { JUNCTION CITY VILLAGE OF } \\
\text { STEVENS POINT CITY OF } \\
\text { WHITING VILLAGE OF } \\
\text { TOTAL }\end{array}$ & $\begin{array}{l}\text { GW } \\
\text { GW } \\
\text { GW } \\
\text { GW }\end{array}$ & $\begin{array}{r}688 \\
499 \\
23607 \\
2025 \\
26819\end{array}$ & $\begin{array}{r}15.00 \\
6.92 \\
404.00 \\
34.90 \\
460.82\end{array}$ & $\begin{array}{r}0.0 \\
0.0 \\
132.00 \\
85.40 \\
217.40\end{array}$ & $\begin{array}{r}5.72 \\
1.06 \\
313.00 \\
2.74 \\
322.52\end{array}$ & $\begin{array}{r}5.57 \\
3.27 \\
591.00 \\
50.57 \\
650.41\end{array}$ & $\begin{array}{r}26.29 \\
11.25 \\
1440.00 \\
173.61 \\
1651.15\end{array}$ \\
\hline PRICE & $\begin{array}{l}\text { PARK FALLS CITY OF } \\
\text { PHILLIPS CITY OF } \\
\text { PRENTICE VILLAGE OF } \\
\text { TOTAL }\end{array}$ & $\begin{array}{l}\text { GW } \\
\text { GW } \\
\text { GW }\end{array}$ & $\begin{array}{r}3130 \\
1566 \\
589 \\
5285\end{array}$ & $\begin{array}{l}48.00 \\
24.90 \\
11.60 \\
34.50\end{array}$ & $\begin{array}{r}40.80 \\
69.10 \\
4.87 \\
114.77\end{array}$ & $\begin{array}{r}31.10 \\
16.80 \\
3.45 \\
51.95\end{array}$ & $\begin{array}{r}52.99 \\
6.64 \\
18.89 \\
78.52\end{array}$ & $\begin{array}{r}173.49 \\
117.44 \\
38.81 \\
329.74\end{array}$ \\
\hline RACINE & $\begin{array}{l}\text { AURL INGTON CITY OF } \\
\text { RACINF CITY OF } \\
\text { STURTFVANT VILLAGE OF } \\
\text { UNIDN GROVE VILLAGE OF } \\
\text { WATERFORD VILLAGE OF } \\
\text { WIND POINT VILLAGE OF } \\
\text { TOTAL }\end{array}$ & $\begin{array}{l}\text { GW } \\
\text { SW } \\
\text { SW } \\
\text { GW } \\
\text { GW } \\
\text { SW }\end{array}$ & $\begin{array}{r}9199 \\
92946 \\
4571 \\
3496 \\
2418 \\
1646 \\
114276\end{array}$ & $\begin{array}{r}175.10 \\
2170.00 \\
77.60 \\
63.30 \\
40.20 \\
58.70 \\
2584.90\end{array}$ & $\begin{array}{r}163.70 \\
3290.00 \\
3.51 \\
137.00 \\
3.50 \\
0.00 \\
3597.71\end{array}$ & $\begin{array}{r}99.70 \\
962.00 \\
25.40 \\
29.40 \\
12.80 \\
17.50 \\
1146.80\end{array}$ & $\begin{array}{r}69.60 \\
1600.00 \\
0.00 \\
83.50 \\
22.91 \\
2.23 \\
1778.24\end{array}$ & $\begin{array}{r}508.10 \\
8022.00 \\
106.51 \\
313.20 \\
79.41 \\
78.43 \\
9107.64\end{array}$ \\
\hline RICHLAND & $\begin{array}{l}\text { CAZENOVIA VILLAGE OF } \\
\text { LONE POCK VILLAGE OF } \\
\text { RICHLAND CENTER CITY OF } \\
\text { VIOLA VILLAGE DF } \\
\text { YUBA VILLAGE OF } \\
\text { TOTAL }\end{array}$ & $\begin{array}{l}\text { GW } \\
\text { GW } \\
\text { GW } \\
\text { GW }\end{array}$ & $\begin{array}{r}315 \\
646 \\
4628 \\
455 \\
69 \\
6113\end{array}$ & $\begin{array}{r}3.46 \\
12.00 \\
82.50 \\
10.00 \\
0.70 \\
108.66\end{array}$ & $\begin{array}{r}0.0 \\
0.0 \\
33.20 \\
0.0 \\
0.49 \\
33.69\end{array}$ & $\begin{array}{r}1.87 \\
1.91 \\
51.40 \\
5.02 \\
0.57 \\
60.77\end{array}$ & $\begin{array}{r}4.42 \\
8.30 \\
57.80 \\
15.45 \\
1.96 \\
87.93\end{array}$ & $\begin{array}{r}9.75 \\
22.21 \\
224.90 \\
30.47 \\
3.73 \\
291.06\end{array}$ \\
\hline Rock & $\begin{array}{l}\text { CLINTON VILLAGE OF } \\
\text { FDGERTON CITY OF } \\
\text { FVANSVILLE CITY OF } \\
\text { FOOTVILLE VILLAGE OF } \\
\text { JANESVILLE CITY OF } \\
\text { MILTON CITY OF } \\
\text { ORFORNVILLE VILLAGE OF } \\
\text { BELOIT CITY OF } \\
\text { TOTAL }\end{array}$ & $\begin{array}{l}\text { GW } \\
\text { GW } \\
\text { GW } \\
\text { GW } \\
\text { GW } \\
\text { GW } \\
\text { GW } \\
\text { GW }\end{array}$ & $\begin{array}{r}1626 \\
4580 \\
3229 \\
744 \\
51500 \\
4836 \\
1146 \\
34711 \\
102372\end{array}$ & $\begin{array}{r}32.30 \\
83.50 \\
59.60 \\
16.40 \\
1530.00 \\
91.60 \\
20.10 \\
854.00 \\
2677.50\end{array}$ & $\begin{array}{r}3.30 \\
29.90 \\
0.00 \\
0.0 \\
685.00 \\
5.47 \\
4.31 \\
631.00 \\
1358.98\end{array}$ & $\begin{array}{r}7.84 \\
36.50 \\
36.50 \\
7.12 \\
737.00 \\
23.00 \\
0.02 \\
346.00 \\
1193.98\end{array}$ & $\begin{array}{r}14.39 \\
235.07 \\
31.10 \\
5.55 \\
753.00 \\
55.43 \\
23.59 \\
1075.30 \\
2193.43\end{array}$ & $\begin{array}{r}57.83 \\
384.97 \\
127.20 \\
29.07 \\
3705.00 \\
165.50 \\
48.02 \\
2906.30 \\
7423.88\end{array}$ \\
\hline RUSK & $\begin{array}{l}\text { ARUCE VILLAGE OF } \\
\text { HAWKINS VILLAGE OF } \\
\text { LADYSMITH CITY DF } \\
\text { WFYERHAEUSER VILLAGE OF } \\
\text { TOTAL }\end{array}$ & $\begin{array}{l}\text { GW } \\
\text { GW } \\
\text { GW } \\
\text { GW }\end{array}$ & $\begin{array}{r}874 \\
396 \\
3716 \\
299 \\
5285\end{array}$ & $\begin{array}{r}12.20 \\
8.00 \\
64.10 \\
4.46 \\
88.76\end{array}$ & $\begin{array}{r}0.29 \\
0.0 \\
17.70 \\
0.00 \\
17.99\end{array}$ & $\begin{array}{r}3.62 \\
3.58 \\
51.40 \\
1.12 \\
59.72\end{array}$ & $\begin{array}{r}13.33 \\
8.21 \\
45.93 \\
2.39 \\
69.86\end{array}$ & $\begin{array}{r}29.44 \\
19.79 \\
179.13 \\
7.97 \\
236.33\end{array}$ \\
\hline ST. CROIX & $\begin{array}{l}\text { RALDWIN VILLAGE OF } \\
\text { GLENWOOD CITY VILLLAGE OF } \\
\text { HAMMOND VILLAGE OF } \\
\text { HUDSON CITY OF } \\
\text { NFW RICHMONO CITY OF } \\
\text { ROBERTS VILLAGE OF } \\
\text { SOMERSET VILLAGE OF } \\
\text { STAR PRAIRIE VILLAGE DF } \\
\text { WOOOVILLE VILLAGE OF } \\
\text { TOTAL }\end{array}$ & $\begin{array}{l}\text { GW } \\
\text { GW } \\
\text { GW } \\
\text { GW } \\
\text { GW } \\
\text { GW } \\
\text { GW } \\
\text { GW } \\
\text { GW }\end{array}$ & $\begin{array}{r}1626 \\
934 \\
994 \\
9088 \\
4321 \\
805 \\
998 \\
465 \\
646 \\
18877\end{array}$ & $\begin{array}{r}21.80 \\
15.00 \\
16.00 \\
147.00 \\
68.60 \\
16.00 \\
13.00 \\
8.87 \\
8.03 \\
314.30\end{array}$ & $\begin{array}{r}0.0 \\
0.0 \\
0.0 \\
12.40 \\
108.00 \\
0.95 \\
2.27 \\
0.0 \\
2.66 \\
126.28\end{array}$ & $\begin{array}{r}58.80 \\
6.02 \\
2.98 \\
59.60 \\
39.60 \\
1.48 \\
6.25 \\
0.34 \\
2.84 \\
177.91\end{array}$ & $\begin{array}{r}4.32 \\
12.11 \\
15.99 \\
75.00 \\
65.30 \\
6.58 \\
15.08 \\
4.52 \\
8.06 \\
206.96\end{array}$ & $\begin{array}{r}84.92 \\
33.13 \\
34.97 \\
294.00 \\
281.50 \\
25.01 \\
36.60 \\
13.74 \\
21.59 \\
825.46\end{array}$ \\
\hline SAUK & $\begin{array}{l}\text { RARABDO CITY OF } \\
\text { LAVALLE VILLAGE OF } \\
\text { LOGANVILLE VILLAGE OF } \\
\text { MFRRIMAC VILLAGE } \\
\text { NORTH FREEDOM VILLLAGE OF } \\
\text { PLAIN VILLAGE OF } \\
\text { PRAIRTE NU SAC CITY OF } \\
\text { REEDSRURG CIITY OF } \\
\text { ROCK SPRINGS VILLAGE OF } \\
\text { SAUK CITY VILLAGE OF } \\
\text { 5PRING GREEN VILLLGE OF } \\
\text { TOTAL }\end{array}$ & $\begin{array}{l}\text { GW } \\
\text { GW } \\
\text { GW } \\
\text { GW } \\
\text { GW } \\
\text { GW } \\
\text { GW } \\
\text { GW } \\
\text { GW } \\
\text { GW } \\
\text { GW }\end{array}$ & $\begin{array}{r}9511 \\
381 \\
189 \\
420 \\
585 \\
707 \\
2306 \\
4821 \\
389 \\
2812 \\
1465 \\
22586\end{array}$ & $\begin{array}{r}160.00 \\
6.49 \\
3.07 \\
5.84 \\
8.55 \\
14.30 \\
44.70 \\
76.70 \\
7.58 \\
54.10 \\
24.60 \\
405.93\end{array}$ & $\begin{array}{c}62.20 \\
0.0 \\
0.0 \\
0.0 \\
0.0 \\
0.0 \\
2.18 \\
245.00 \\
0.0 \\
0.0 \\
0.53 \\
309.91\end{array}$ & $\begin{array}{r}85.30 \\
2.31 \\
1.10 \\
2.33 \\
3.90 \\
7.67 \\
15.20 \\
50.00 \\
1.59 \\
62.40 \\
9.74 \\
241.54\end{array}$ & $\begin{array}{r}84.60 \\
3.30 \\
0.56 \\
2.54 \\
8.80 \\
10.44 \\
35.43 \\
44.90 \\
2.23 \\
31.00 \\
24.03 \\
247.82\end{array}$ & $\begin{array}{r}392.10 \\
12.10 \\
4.73 \\
10.71 \\
21.25 \\
32.41 \\
97.51 \\
416.60 \\
11.40 \\
147.50 \\
58.90 \\
1205.20\end{array}$ \\
\hline SAWYER & $\begin{array}{l}\text { HAYWARD VILLAGE OF } \\
\text { RAOISSON VILLAGE OF } \\
\text { WINTER VILLAGE OF } \\
\text { TOTAL }\end{array}$ & $\begin{array}{l}\text { GW } \\
\text { GW } \\
\text { GW }\end{array}$ & $\begin{array}{r}1595 \\
242 \\
352 \\
2189\end{array}$ & $\begin{array}{r}20.30 \\
.00 \\
3.55 \\
27.85\end{array}$ & $\begin{array}{l}2.30 \\
0.0 \\
0.29 \\
2.59\end{array}$ & $\begin{array}{r}39.20 \\
2.00 \\
4.00 \\
45.20\end{array}$ & $\begin{array}{r}30.71 \\
1.32 \\
7.09 \\
39.12\end{array}$ & $\begin{array}{r}92.51 \\
7.32 \\
14.93 \\
114.76\end{array}$ \\
\hline
\end{tabular}




\section{Table 1.--Municipal water use in 1979--Continued}

\begin{tabular}{|c|c|c|c|c|c|c|c|c|}
\hline \multirow[b]{2}{*}{ COUNTY } & \multirow[b]{2}{*}{ MUNICIPALITY } & \multirow[b]{2}{*}{ SOURCE } & \multirow[b]{2}{*}{ POPULATION } & \multirow[b]{2}{*}{ RESIDENTIAL } & \multicolumn{4}{|c|}{ WATER DELIVERIES IN MILLIONS OF GALLONS } \\
\hline & & & & & INOUSTRI AL & COMMERCIAL & OTHEK & TOTAL \\
\hline SHAWANO & $\begin{array}{l}\text { RIRNANHOOD VILLAGE DF } \\
\text { BONDUEL VILLAGE OF } \\
\text { BOWLER VILLAGE OF } \\
\text { GRESHAM VILLAGE OF } \\
\text { MATIONN VILLAGE OF } \\
\text { SHAWANO CTIY OF } \\
\text { TIGERTON VILLAGE OF } \\
\text { WITTENGURG VILLAGE OF } \\
\text { TOTAL }\end{array}$ & $\begin{array}{l}\text { GW } \\
\text { GW } \\
\text { GW } \\
\text { GW } \\
\text { GW } \\
\text { GW } \\
\text { GW } \\
\text { GW }\end{array}$ & $\begin{array}{r}707 \\
1168 \\
297 \\
510 \\
344 \\
5881 \\
811 \\
993 \\
11711\end{array}$ & $\begin{array}{r}6.43 \\
19.20 \\
4.52 \\
7.87 \\
4.24 \\
126.00 \\
9.35 \\
12.20 \\
189.81\end{array}$ & $\begin{array}{r}0.08 \\
54.00 \\
0.0 \\
0.10 \\
2.13 \\
92.00 \\
2.07 \\
0.05 \\
151.03\end{array}$ & $\begin{array}{r}5.49 \\
9.65 \\
0.67 \\
3.36 \\
0.89 \\
144.00 \\
5.27 \\
12.60 \\
181.93\end{array}$ & $\begin{array}{r}7.40 \\
18.98 \\
5.44 \\
4.50 \\
5.45 \\
115.40 \\
16.70 \\
16.15 \\
190.42\end{array}$ & $\begin{array}{r}19.40 \\
101.83 \\
10.63 \\
15.83 \\
13.11 \\
478.00 \\
33.39 \\
41.00 \\
713.19\end{array}$ \\
\hline SHEBOYGAN & 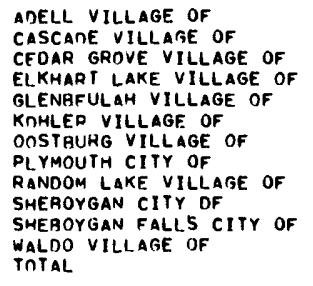 & $\begin{array}{l}G W \\
G W \\
G W \\
G W \\
G W \\
S W \\
G W \\
G W \\
G W \\
S W \\
S W \\
G W\end{array}$ & $\begin{array}{r}554 \\
630 \\
1465 \\
994 \\
488 \\
1697 \\
1662 \\
6139 \\
1321 \\
48436 \\
5253 \\
442 \\
69081\end{array}$ & $\begin{array}{r}8.32 \\
9.17 \\
29.30 \\
20.40 \\
7.04 \\
37.50 \\
33.80 \\
106.00 \\
25.50 \\
1070.00 \\
81.70 \\
6.34 \\
1435.07\end{array}$ & $\begin{array}{r}1.30 \\
0.0 \\
43.00 \\
3.24 \\
0.0 \\
34.20 \\
5.02 \\
364.00 \\
88.10 \\
1650.00 \\
427.00 \\
9.27 \\
2625.13\end{array}$ & $\begin{array}{r}2.58 \\
2.06 \\
3.74 \\
9.37 \\
1.62 \\
11.60 \\
9.60 \\
81.50 \\
9.64 \\
379.00 \\
16.40 \\
0.39 \\
527.54\end{array}$ & $\begin{array}{r}2.46 \\
0.54 \\
8.28 \\
33.49 \\
6.54 \\
13.70 \\
6.36 \\
99.30 \\
16.23 \\
662.00 \\
77.00 \\
11.86 \\
937.76\end{array}$ & $\begin{array}{r}14.66 \\
11.77 \\
84.36 \\
66.50 \\
15.20 \\
97.00 \\
54.78 \\
650.80 \\
139.47 \\
3761.001 \\
602.10 \\
27.86 \\
5525.49\end{array}$ \\
\hline TAYLOR & $\begin{array}{l}\text { GILMAN VILLAGE OF } \\
\text { MEOFORO VILLAGE OF } \\
\text { RIB LAKE VILLAGE OF } \\
\text { TOTAL }\end{array}$ & $\begin{array}{l}\text { GW } \\
\text { GW } \\
\text { GW }\end{array}$ & $\begin{array}{r}422 \\
4127 \\
887 \\
5436\end{array}$ & $\begin{array}{l}5.00 \\
66.70 \\
9.77 \\
81.47\end{array}$ & $\begin{array}{r}5.24 \\
53.00 \\
1.15 \\
59.39\end{array}$ & $\begin{array}{l}2.85 \\
15.80 \\
4.22 \\
22.87\end{array}$ & $\begin{array}{l}6.57 \\
63.70 \\
5.14 \\
75.41\end{array}$ & $\begin{array}{l}19.66 \\
199.20 \\
20.28 \\
239.14\end{array}$ \\
\hline TREMPEALEAU & $\begin{array}{l}\text { ARCAOIA CIIY OF } \\
\text { RLAIR CIIY OF } \\
\text { ELEVA VILLAGE OF } \\
\text { ETIRICK VILLAGE OF } \\
\text { GALESVILLE CITY OF } \\
\text { INDEPFNOENCE CITY OF } \\
\text { OSSEO CIYY OF } \\
\text { PIGEON FALLS VILLAGE OF } \\
\text { STRUM VILLAGE OF } \\
\text { IREMPFALEAU VILLAGE OF } \\
\text { WHITEHAL CITY OF } \\
\text { TOTAL }\end{array}$ & $\begin{array}{l}G W \\
G W \\
G W \\
G W \\
G W \\
G W \\
G W \\
G W \\
G W \\
G W \\
G W\end{array}$ & $\begin{array}{r}2305 \\
1144 \\
633 \\
478 \\
1291 \\
1089 \\
1478 \\
284 \\
872 \\
897 \\
1558 \\
12029\end{array}$ & $\begin{array}{l}27.40 \\
16.40 \\
9.42 \\
10.20 \\
20.10 \\
16.00 \\
21.20 \\
4.67 \\
12.60 \\
14.20 \\
23.60 \\
175.79\end{array}$ & $\begin{array}{c}191.00 \\
15.20 \\
0.0 \\
1.91 \\
26.30 \\
0.34 \\
0.0 \\
0.0 \\
0.0 \\
0.0 \\
21.30 \\
166.05\end{array}$ & $\begin{array}{r}25.20 \\
6.56 \\
2.22 \\
4.26 \\
10.40 \\
6.94 \\
11.90 \\
1.30 \\
5.51 \\
3.20 \\
16.10 \\
93.39\end{array}$ & $\begin{array}{r}22.98 \\
19.10 \\
4.48 \\
5.40 \\
46.39 \\
13.19 \\
19.60 \\
2.32 \\
5.17 \\
8.39 \\
22.46 \\
169.48\end{array}$ & $\begin{array}{r}176.54 \\
57.26 \\
16.12 \\
21.77 \\
103.19 \\
36.47 \\
52.50 \\
0.29 \\
23.28 \\
25.79 \\
83.46 \\
604.70\end{array}$ \\
\hline VERNON & $\begin{array}{l}\text { CHASEAURG VILLAGE OF } \\
\text { COON VALLEY VILLAGE OF } \\
\text { GFNOA VILLAGE OF } \\
\text { HILLSRORO CITY OF } \\
\text { LAFARGE VILLAGE OF } \\
\text { DNTARIO VILLAGE OF } \\
\text { READSTOWN VILLAGE OF } \\
\text { STODOARO VILLAGE OF } \\
\text { VIROQIIA CITY OF } \\
\text { WESTBY CITY OF } \\
\text { TOTAL }\end{array}$ & $\begin{array}{l}G W \\
G W \\
G W \\
G W \\
G W \\
G W \\
G W \\
G W \\
G W\end{array}$ & $\begin{array}{r}272 \\
750 \\
313 \\
1202 \\
668 \\
390 \\
323 \\
911 \\
3595 \\
17 A 4 \\
10208\end{array}$ & $\begin{array}{r}3.47 \\
12.20 \\
4.77 \\
20.40 \\
9.90 \\
5.35 \\
5.02 \\
14.10 \\
63.70 \\
28.40 \\
167.31\end{array}$ & $\begin{array}{l}5.32 \\
0.0 \\
0.00 \\
14.00 \\
5.70 \\
0.14 \\
2.71 \\
0.0 \\
3.92 \\
41.80 \\
73.59\end{array}$ & $\begin{array}{r}1.11 \\
9.62 \\
2.47 \\
9.38 \\
3.62 \\
1.24 \\
6.58 \\
3.57 \\
50.70 \\
9.16 \\
97.45\end{array}$ & $\begin{array}{r}3.69 \\
5.67 \\
3.46 \\
19.90 \\
10.17 \\
4.97 \\
4.78 \\
5.23 \\
57.40 \\
23.81 \\
139.07\end{array}$ & $\begin{array}{l}13.59 \\
27.49 \\
10.70 \\
63.68 \\
29.39 \\
11.70 \\
19.09 \\
22.90 \\
175.72 \\
103.17 \\
477.42\end{array}$ \\
\hline VILAS & $\begin{array}{l}\text { EAGLE NIVER CITY OF } \\
\text { TDTAL }\end{array}$ & GW & $\begin{array}{l}1421 \\
1421\end{array}$ & $\begin{array}{l}22.20 \\
22.20\end{array}$ & $\begin{array}{l}0.0 \\
0.0\end{array}$ & $\begin{array}{l}64.10 \\
64.10\end{array}$ & $\begin{array}{l}39.72 \\
39.72\end{array}$ & $\begin{array}{l}126.02 \\
126.02\end{array}$ \\
\hline WALWORTH & 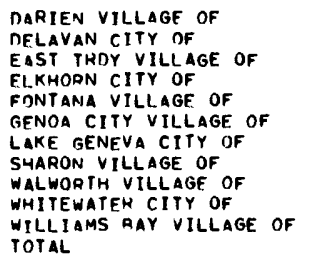 & $\begin{array}{l}\text { GW } \\
G W \\
G W \\
G W \\
G W \\
G W \\
G W \\
G W \\
G W \\
G W \\
\text { GW }\end{array}$ & $\begin{array}{r}1143 \\
5715 \\
2509 \\
4598 \\
1823 \\
1266 \\
5565 \\
1339 \\
1682 \\
10626 \\
1646 \\
37912\end{array}$ & $\begin{array}{r}18.80 \\
108.00 \\
40.60 \\
83.60 \\
55.40 \\
17.90 \\
123.00 \\
23.30 \\
27.80 \\
110.00 \\
47.00 \\
655.40\end{array}$ & $\begin{array}{r}1.12 \\
58.70 \\
140.00 \\
18.90 \\
0.0 \\
1.33 \\
59.80 \\
5.25 \\
33.10 \\
186.00 \\
0.00 \\
504.20\end{array}$ & $\begin{array}{r}4.58 \\
50.60 \\
25.80 \\
46.20 \\
44.10 \\
10.30 \\
168.00 \\
4.51 \\
14.00 \\
94.10 \\
20.80 \\
482.99\end{array}$ & $\begin{array}{r}6.27 \\
46.40 \\
39.76 \\
75.00 \\
44.35 \\
9.64 \\
152.30 \\
12.70 \\
43.14 \\
160.00 \\
33.16 \\
622.72\end{array}$ & $\begin{array}{r}30.77 \\
263.70 \\
246.16 \\
223.70 \\
143.85 \\
39.17 \\
503.10 \\
45.76 \\
118.74 \\
550.10 \\
100.96 \\
2265.30\end{array}$ \\
\hline WASHAURN & $\begin{array}{l}\text { RTRCHWOON VILLAGE OF } \\
\text { MINONG VILLAGE OF } \\
\text { SHELL LAKE CIYY OF } \\
\text { SPOONER CITY OF } \\
\text { TOTAL }\end{array}$ & $\begin{array}{l}\text { GW } \\
G W \\
G W \\
G W\end{array}$ & $\begin{array}{r}399 \\
528 \\
1113 \\
2457 \\
4497\end{array}$ & $\begin{array}{r}6.48 \\
8.78 \\
15.10 \\
44.00 \\
74.36\end{array}$ & $\begin{array}{r}2.24 \\
7.22 \\
0.71 \\
0.61 \\
10.78\end{array}$ & $\begin{array}{r}3.41 \\
9.32 \\
12.20 \\
17.40 \\
42.33\end{array}$ & $\begin{array}{r}2.67 \\
49.40 \\
24.98 \\
60.90 \\
137.95\end{array}$ & $\begin{array}{r}14.80 \\
74.72 \\
52.99 \\
122.91 \\
265.42\end{array}$ \\
\hline WASHINGTON & $\begin{array}{l}\text { GERMANTOWN VILLAGE OF } \\
\text { HARTFNRO CITY OF } \\
\text { JACKSON VILLAGE OF } \\
\text { KEWASKUM VILLAGE OF } \\
\text { SLINGFN VILLAGE OF } \\
\text { WEST AEND CITY OF } \\
\text { TOTAL }\end{array}$ & $\begin{array}{l}G W \\
G W \\
G W \\
G W \\
G W \\
G W\end{array}$ & $\begin{array}{r}4400 \\
7599 \\
2065 \\
2560 \\
1759 \\
23110 \\
41493\end{array}$ & $\begin{array}{r}77.80 \\
126.00 \\
20.60 \\
43.50 \\
29.00 \\
492.00 \\
788.90\end{array}$ & $\begin{array}{r}16.20 \\
218.00 \\
32.30 \\
96.80 \\
39.00 \\
489.00 \\
891.30\end{array}$ & $\begin{array}{r}43.30 \\
65.90 \\
26.10 \\
11.20 \\
12.90 \\
129.00 \\
288.40\end{array}$ & $\begin{array}{r}37.10 \\
61.50 \\
4.03 \\
6.05 \\
18.06 \\
308.20 \\
434.94\end{array}$ & $\begin{array}{r}174.40 \\
471.40 \\
83.03 \\
157.55 \\
98.96 \\
1418.20 \\
2403.54\end{array}$ \\
\hline WAUKESHA & $\begin{array}{l}\text { AROOKF IELD CITY OF } \\
\text { AIIITER VILLAGE OF } \\
\text { MOUSMAN VILLAGE OF } \\
\text { EAGE VILLAGE OF } \\
\text { HARTLANO VILLAGE OF } \\
\text { MFNMONEE FALLS VILLG OF } \\
\text { MIJKWONAGO VILLAGE OFF } \\
\text { NEW BFRLIN CIYY OF } \\
\text { NCONOMOWOC CITY OF } \\
\text { PFWAUKEE VILLAGE OF }\end{array}$ & $\begin{array}{l}G W \\
G W \\
G W \\
G W \\
G W \\
G W \\
G W \\
G W \\
G W\end{array}$ & $\begin{array}{r}36061 \\
2102 \\
1051 \\
1011 \\
5513 \\
32922 \\
4300 \\
33913 \\
10898 \\
4733\end{array}$ & $\begin{array}{r}324.00 \\
38.10 \\
22.70 \\
18.50 \\
107.00 \\
418.00 \\
87.80 \\
242.00 \\
198.00 \\
62.50\end{array}$ & $\begin{array}{r}16.20 \\
62.50 \\
0.20 \\
0.0 \\
1.45 \\
201.00 \\
1.98 \\
252.00 \\
40.10 \\
75.40\end{array}$ & $\begin{array}{r}92.50 \\
49.90 \\
2.60 \\
0.88 \\
50.90 \\
102.00 \\
13.10 \\
25.80 \\
161.00 \\
55.00\end{array}$ & $\begin{array}{r}83.62 \\
24.41 \\
7.96 \\
6.27 \\
235.40 \\
196.70 \\
41.50 \\
63.90 \\
105.62 \\
30.60\end{array}$ & $\begin{array}{r}516.32 \\
174.91 \\
33.46 \\
25.65 \\
394.75 \\
917.70 \\
144.38 \\
583.70 \\
504.72 \\
223.50\end{array}$ \\
\hline
\end{tabular}




\section{Table 1.--Municipal water use in 1979--Continued}

\begin{tabular}{|c|c|c|c|c|c|c|c|c|}
\hline & & & & & WATER OEL & IVERIES IN & LIONS OF & \\
\hline COIINTY & MUNICIPALITY & SOURCE & POPULATION & RESIDENTIAL & INDUSTRIAL & COMMERC I AL & OTHER & TOTAL \\
\hline WAUKESHA & $\begin{array}{l}\text { SUSSEX VILLAGE OF } \\
\text { WAUKESHA CITY OF } \\
\text { TOTAL. }\end{array}$ & $\begin{array}{l}\text { GW } \\
\text { GW }\end{array}$ & $\begin{array}{r}4297 \\
52770 \\
189571\end{array}$ & $\begin{array}{r}76.90 \\
1010.00 \\
2605.50\end{array}$ & $\begin{array}{r}0.25 \\
1610.00 \\
2261.08\end{array}$ & $\begin{array}{r}6.32 \\
340.00 \\
900.00\end{array}$ & $\begin{array}{r}20.60 \\
565.00 \\
1381.58\end{array}$ & $\begin{array}{r}104.07 \\
3525.00 \\
7148.16\end{array}$ \\
\hline WAUPACA & $\begin{array}{l}\text { CLINTONVILLE CITY OF } \\
\text { FMBARRASS VILLAGE OF } \\
\text { IOLA VILLLGE OF } \\
\text { MANAWA CITY OF } \\
\text { MARION CITY OF } \\
\text { NEW LANOON CITY OF } \\
\text { WAUPACA CITY OF } \\
\text { WEYAUWEGA CITY OF } \\
\text { TOTAL. }\end{array}$ & $\begin{array}{l}\text { GW } \\
\text { GW } \\
\text { GW } \\
\text { GW } \\
\text { GW } \\
\text { GW } \\
\text { GW } \\
\text { GW }\end{array}$ & $\begin{array}{r}4443 \\
546 \\
1011 \\
1179 \\
1315 \\
6283 \\
4645 \\
1608 \\
21030\end{array}$ & $\begin{array}{r}79.80 \\
5.82 \\
15.40 \\
21.20 \\
19.90 \\
111.00 \\
74.40 \\
29.80 \\
357.32\end{array}$ & $\begin{array}{r}45.40 \\
0.13 \\
0.0 \\
22.10 \\
12.20 \\
304.00 \\
628.00 \\
72.20 \\
1084.03\end{array}$ & $\begin{array}{r}63.80 \\
5.24 \\
9.37 \\
7.32 \\
8.12 \\
34.90 \\
48.70 \\
19.20 \\
196.65\end{array}$ & $\begin{array}{r}16.80 \\
4.20 \\
11.55 \\
16.00 \\
56.33 \\
48.80 \\
26.10 \\
38.61 \\
218.39\end{array}$ & $\begin{array}{r}205.80 \\
15.39 \\
36.32 \\
66.62 \\
96.55 \\
498.70 \\
777.20 \\
159.81 \\
1856.39\end{array}$ \\
\hline WAUSHARA & $\begin{array}{l}\text { COLOMA VILLAGE OF } \\
\text { HANCOCK VILLAGE OF } \\
\text { PLAINFIELD VILLAGE OF } \\
\text { RFDGRANITE VILLAGE OF } \\
\text { TOTAL }\end{array}$ & $\begin{array}{l}\text { GW } \\
\text { GW } \\
\text { GW } \\
\text { GW }\end{array}$ & $\begin{array}{r}341 \\
417 \\
707 \\
797 \\
2262\end{array}$ & $\begin{array}{r}7.28 \\
9.08 \\
30.50 \\
11.00 \\
57.06\end{array}$ & $\begin{array}{l}0.0 \\
1.27 \\
3.60 \\
3.07 \\
7.94\end{array}$ & $\begin{array}{r}2.64 \\
2.47 \\
0.61 \\
5.25 \\
10.97\end{array}$ & $\begin{array}{r}11.88 \\
5.74 \\
5.10 \\
2.30 \\
25.02\end{array}$ & $\begin{array}{r}21.80 \\
18.56 \\
39.81 \\
21.62 \\
101.79\end{array}$ \\
\hline WINNERAGO & $\begin{array}{l}\text { MENASHA CITY OF } \\
\text { NFENAH CITY OF } \\
\text { OMRO CITY OF } \\
\text { OSHKOSH CITY OF } \\
\text { WINNECONNE VILLAGE OF } \\
\text { TOTAL }\end{array}$ & $\begin{array}{l}\text { SW } \\
5 W \\
\text { GW } \\
\text { SW } \\
\text { GW }\end{array}$ & $\begin{array}{r}15057 \\
23765 \\
2753 \\
50231 \\
2014 \\
93820\end{array}$ & $\begin{array}{r}280.00 \\
452.00 \\
38.30 \\
931.00 \\
35.30 \\
1736.60\end{array}$ & $\begin{array}{r}981.00 \\
653.00 \\
6.27 \\
554.00 \\
.6 .70 \\
2200.97\end{array}$ & $\begin{array}{r}78.50 \\
128.00 \\
16.70 \\
246.00 \\
14.00 \\
483.20\end{array}$ & $\begin{array}{r}390.00 \\
422.40 \\
19.00 \\
524.00 \\
14.57 \\
1369.97\end{array}$ & $\begin{array}{r}1729.50 * \\
1655.40 \\
80.27 \\
2255.00 \\
70.57 \\
5790.73\end{array}$ \\
\hline woon & $\begin{array}{l}\text { AIRON VILLAGE OF } \\
\text { MARSHFIELO CITY OF } \\
\text { NEKOOSA CITY OF } \\
\text { PITTSVILLE CITY OF } \\
\text { PORT FOWARDS VILLAGE OF } \\
\text { VFSPER VILLAGE OF } \\
\text { WISCONSIN RAPIOS CITY OF } \\
\text { TOTAL }\end{array}$ & $\begin{array}{l}\text { GW } \\
\text { GW } \\
\text { GW } \\
\text { GW } \\
\text { GW } \\
\text { GW } \\
\text { GW }\end{array}$ & $\begin{array}{r}736 \\
17553 \\
2564 \\
793 \\
2147 \\
488 \\
18266 \\
42547\end{array}$ & $\begin{array}{r}12.20 \\
279.00 \\
47.70 \\
8.24 \\
39.80 \\
6.61 \\
308.00 \\
701.55\end{array}$ & $\begin{array}{r}13.70 \\
210.00 \\
42.50 \\
1.46 \\
75.60 \\
12.00 \\
232.00 \\
587.26\end{array}$ & $\begin{array}{r}0.16 \\
187.00 \\
10.20 \\
4.41 \\
25.40 \\
1.60 \\
170.00 \\
398.77\end{array}$ & $\begin{array}{r}5.97 \\
206.08 \\
36.40 \\
11.08 \\
6.41 \\
11.73 \\
259.20 \\
536.87\end{array}$ & $\begin{array}{r}32.03 \\
882.08 \\
136.80 \\
25.19 \\
147.21 \\
31.94 \\
969.20 \\
2224.45\end{array}$ \\
\hline
\end{tabular}

* water nelivfries for wholesaling to other communities are not included in this total. 


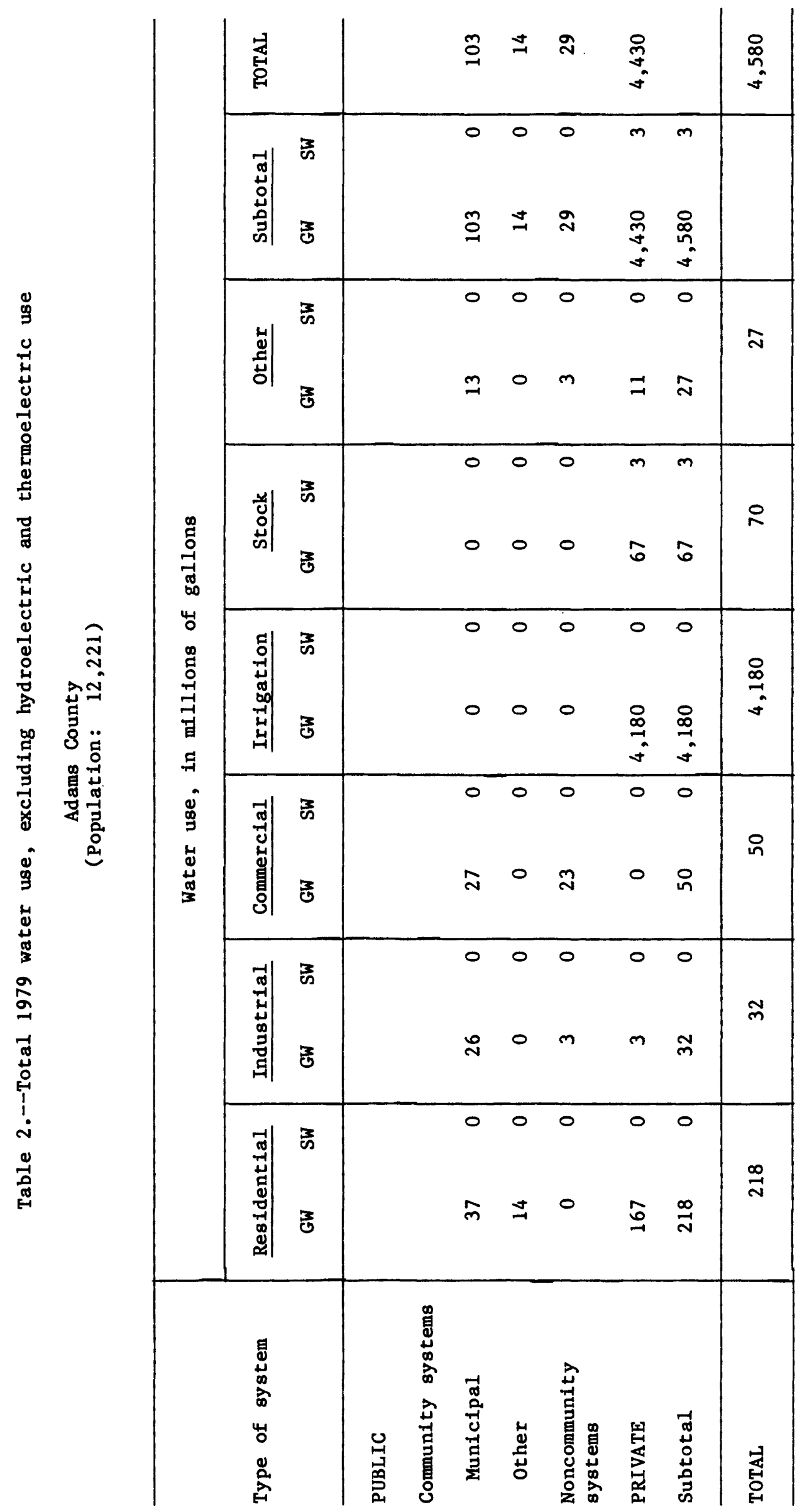




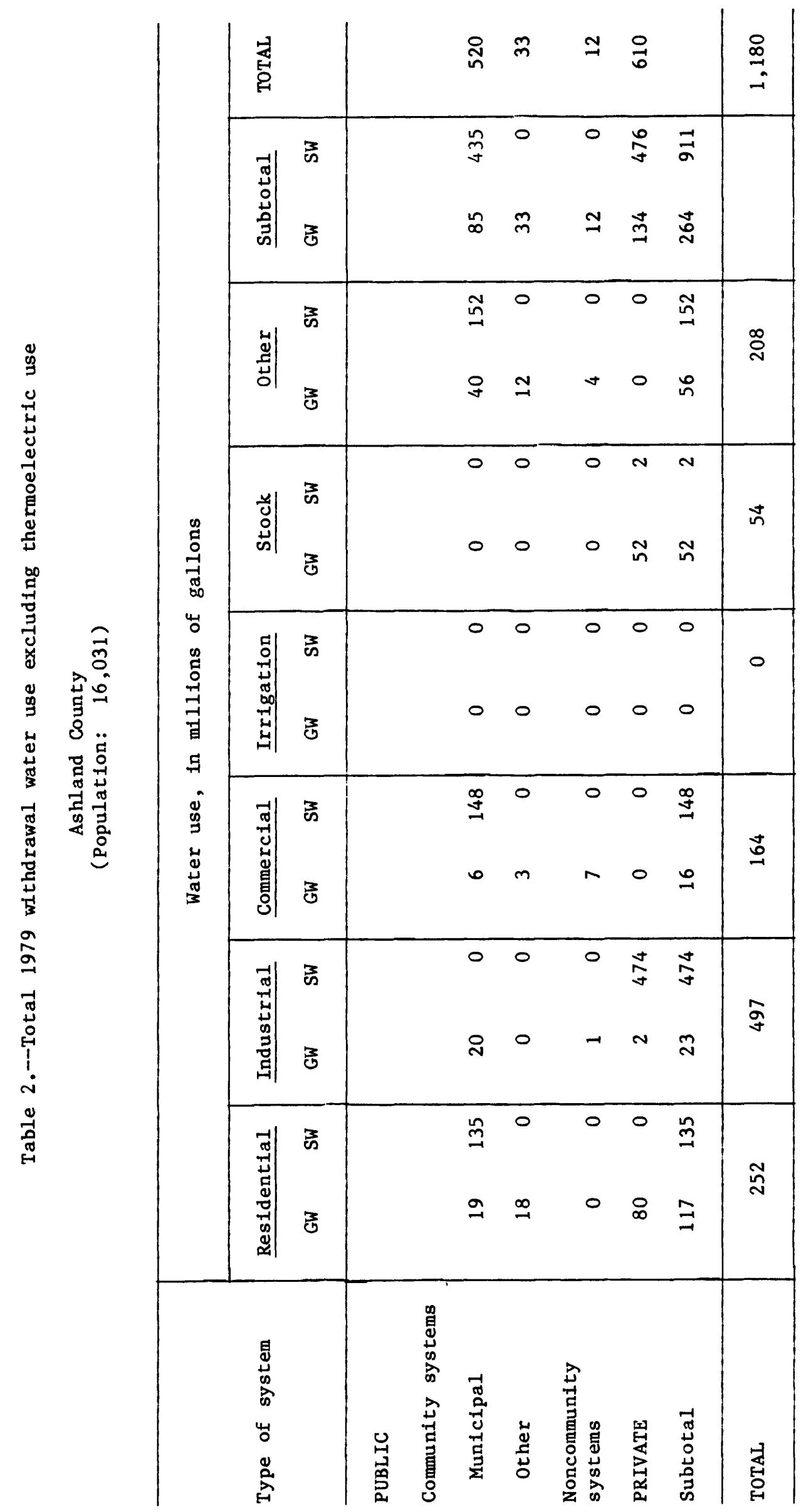




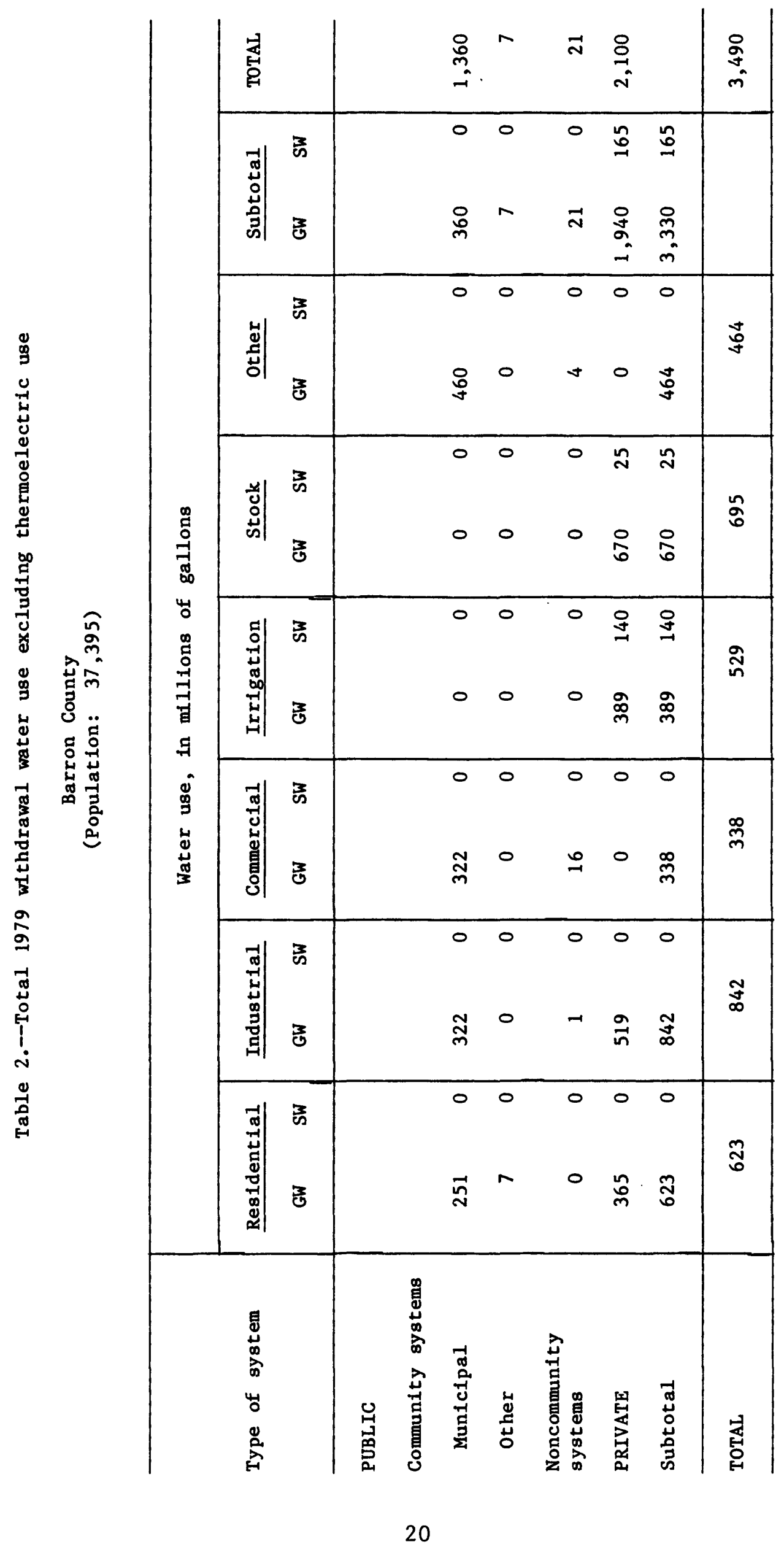




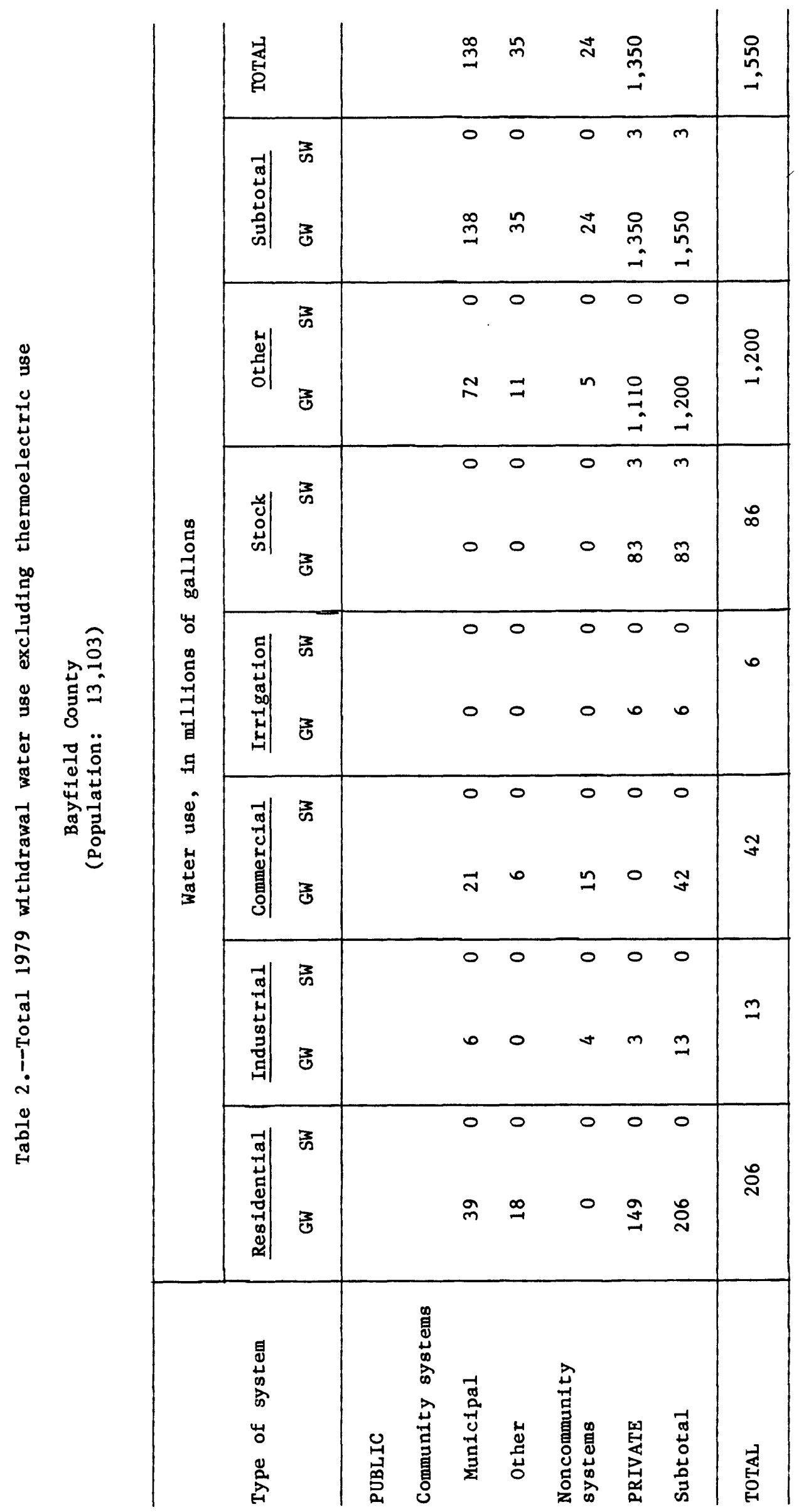




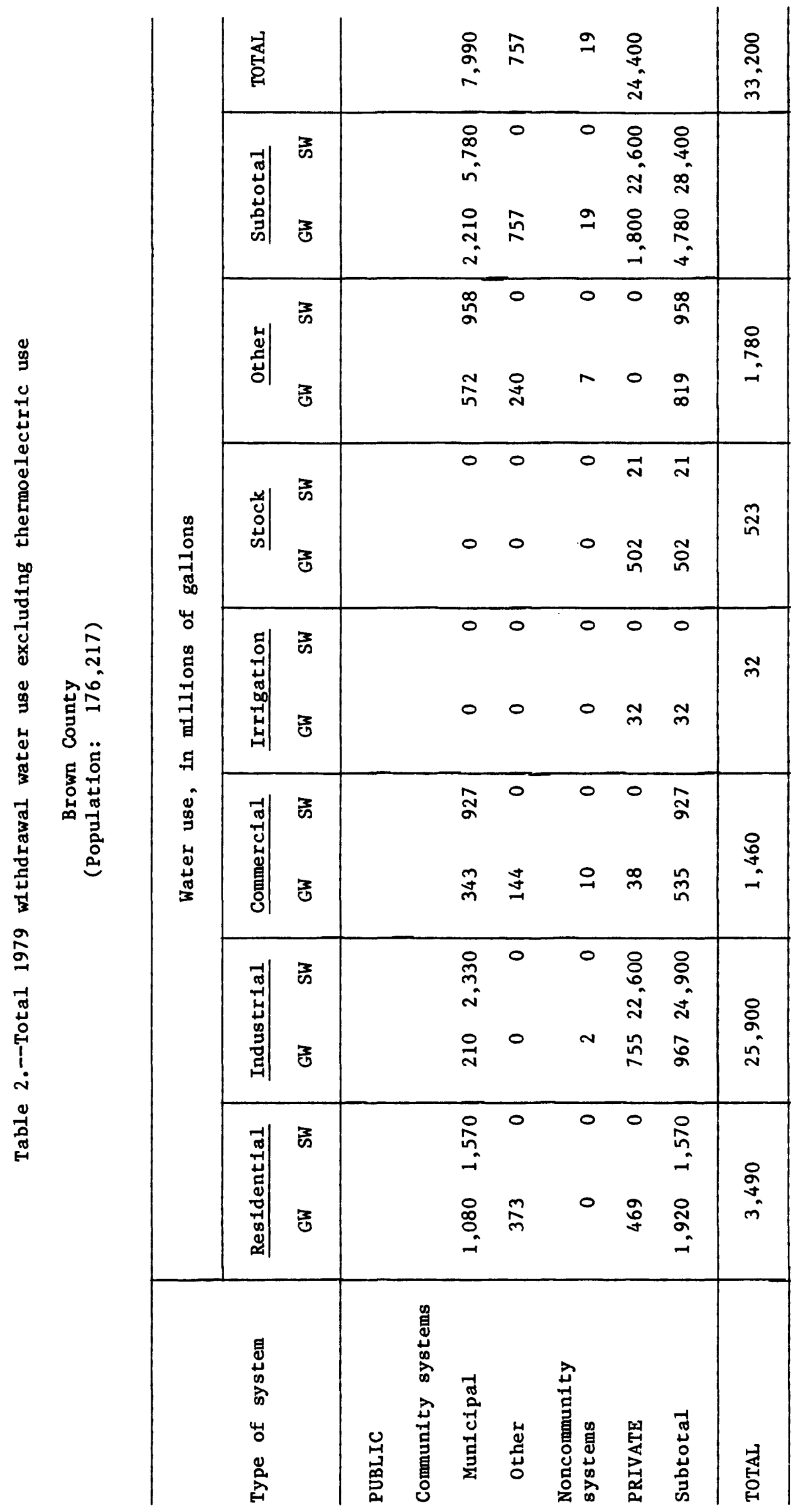




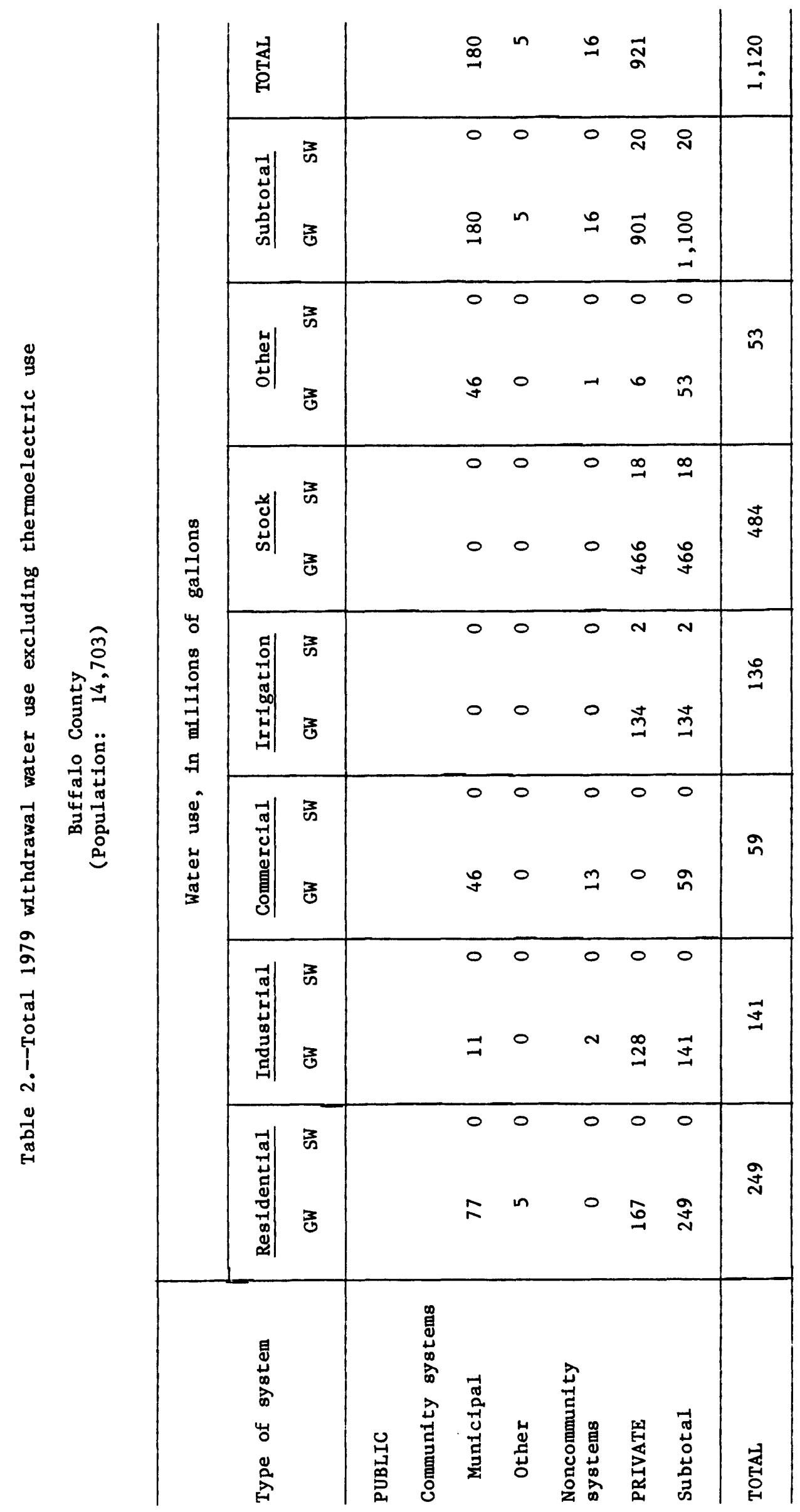




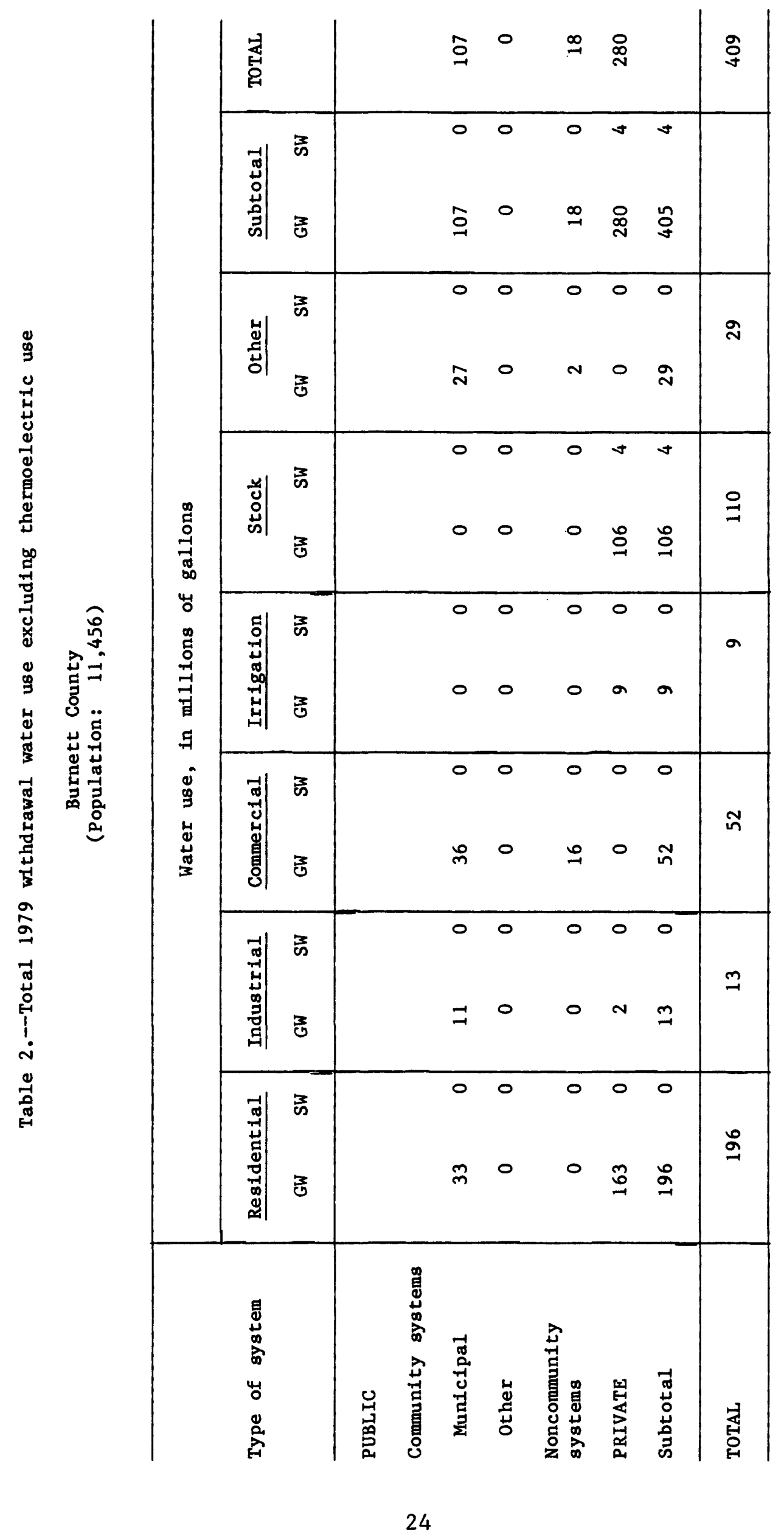




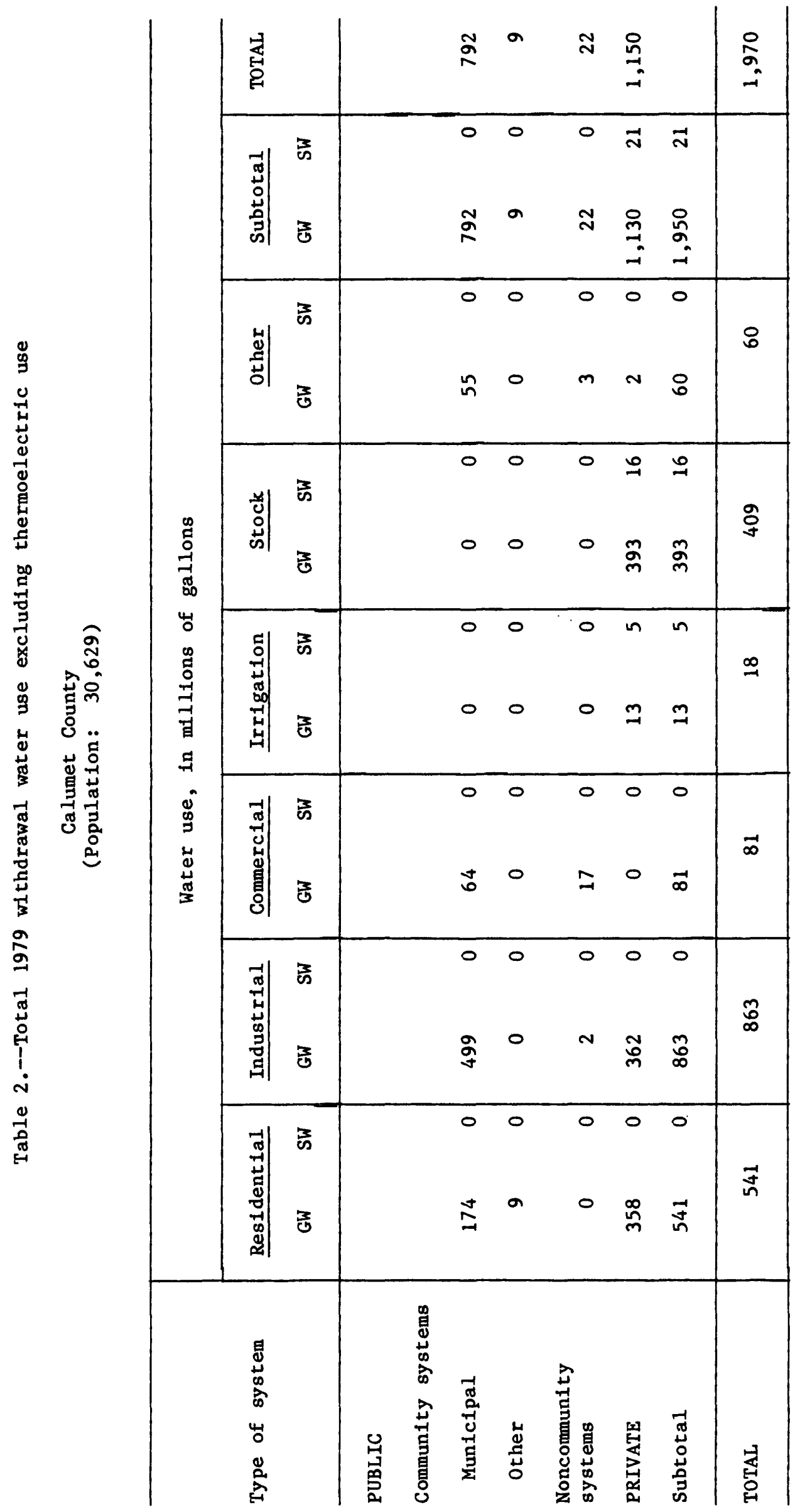




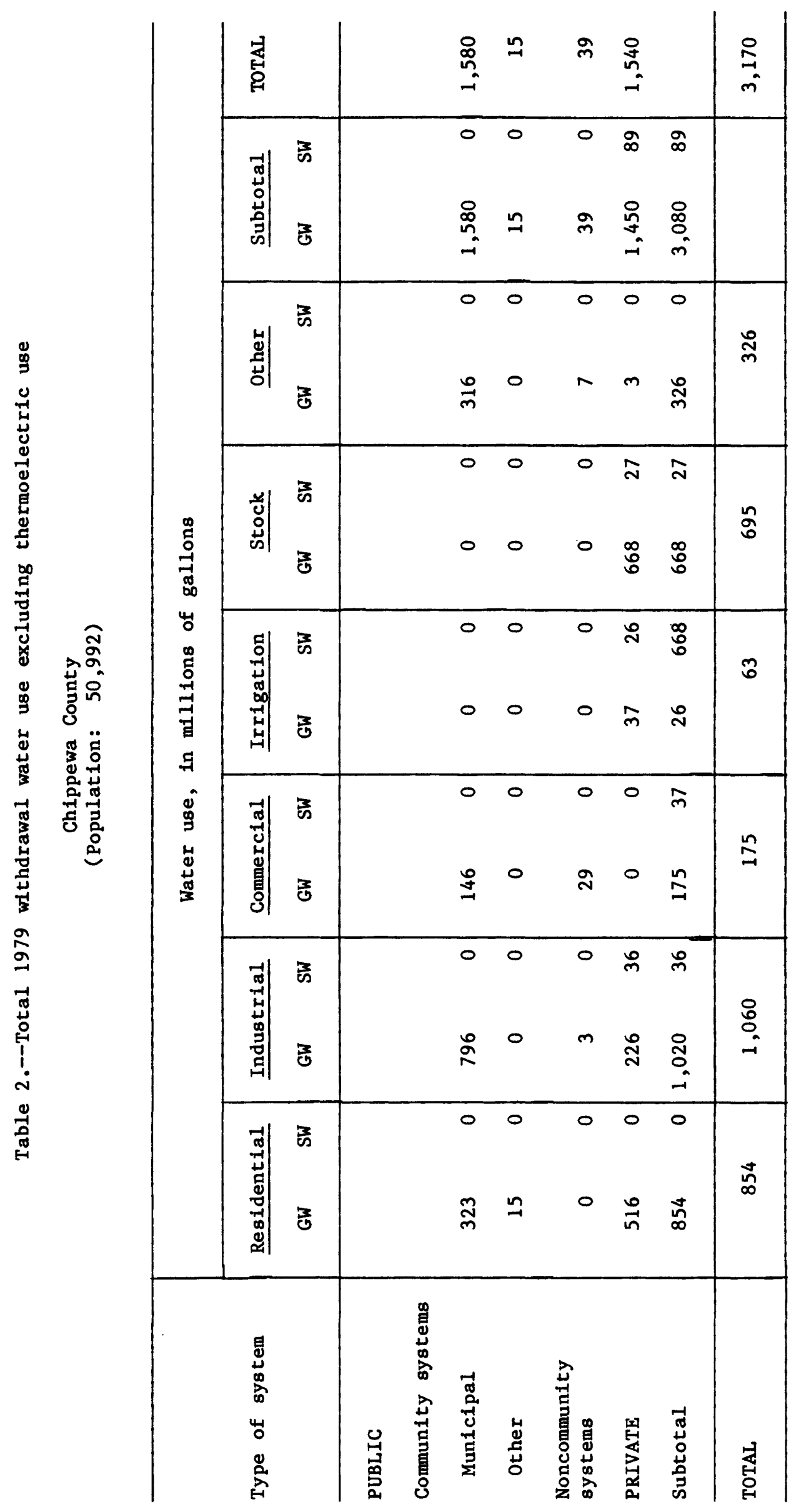




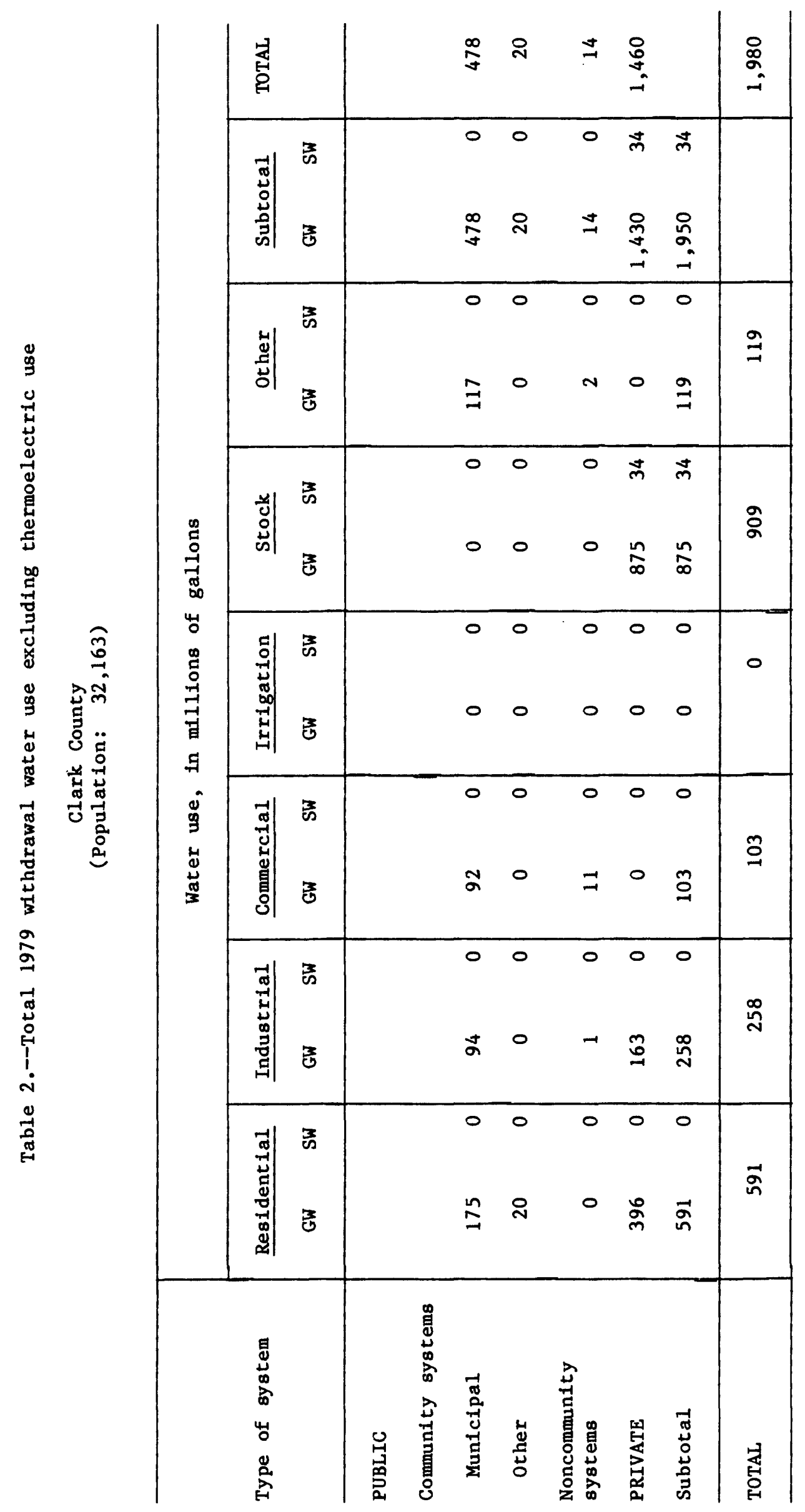




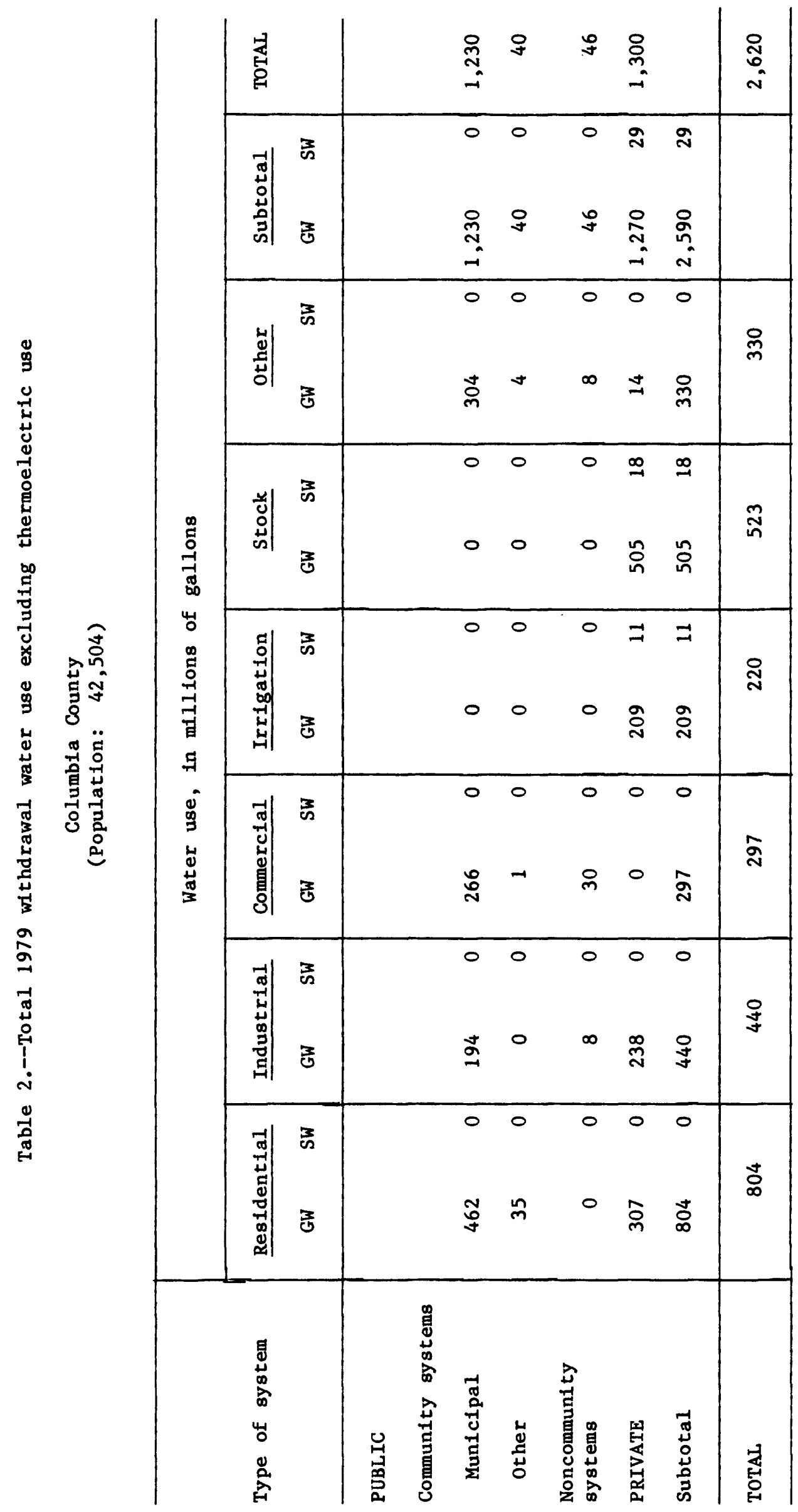




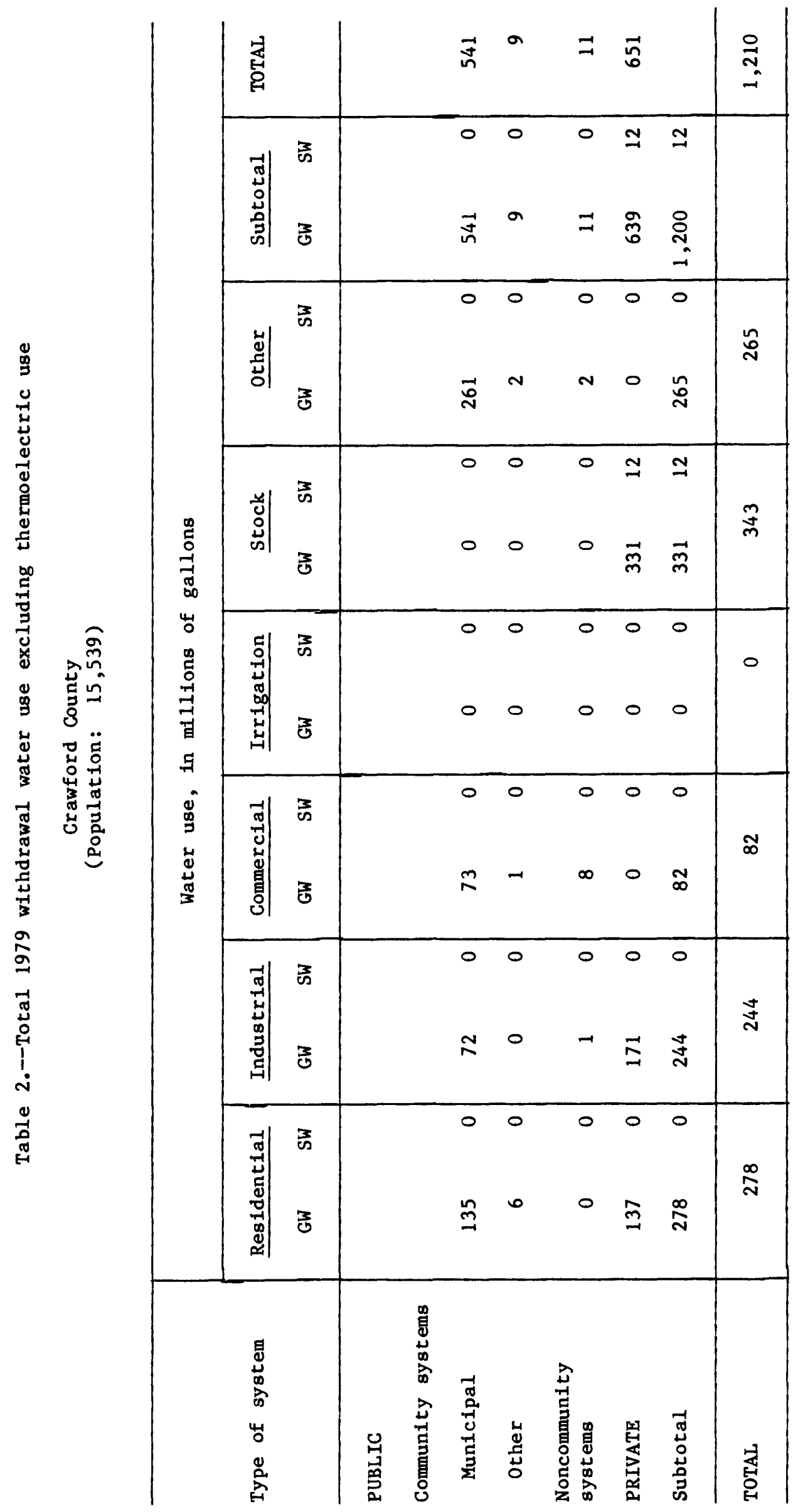




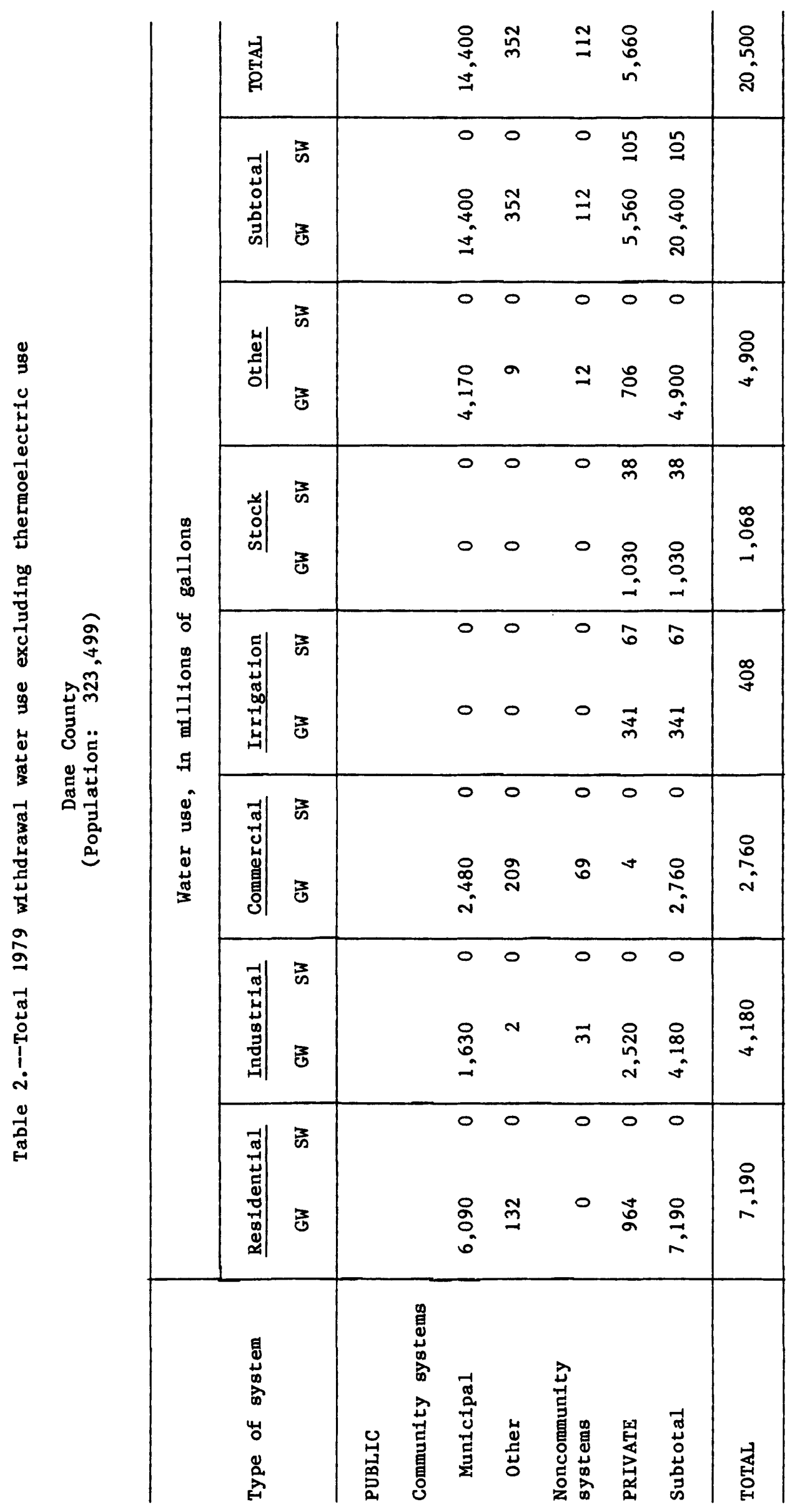




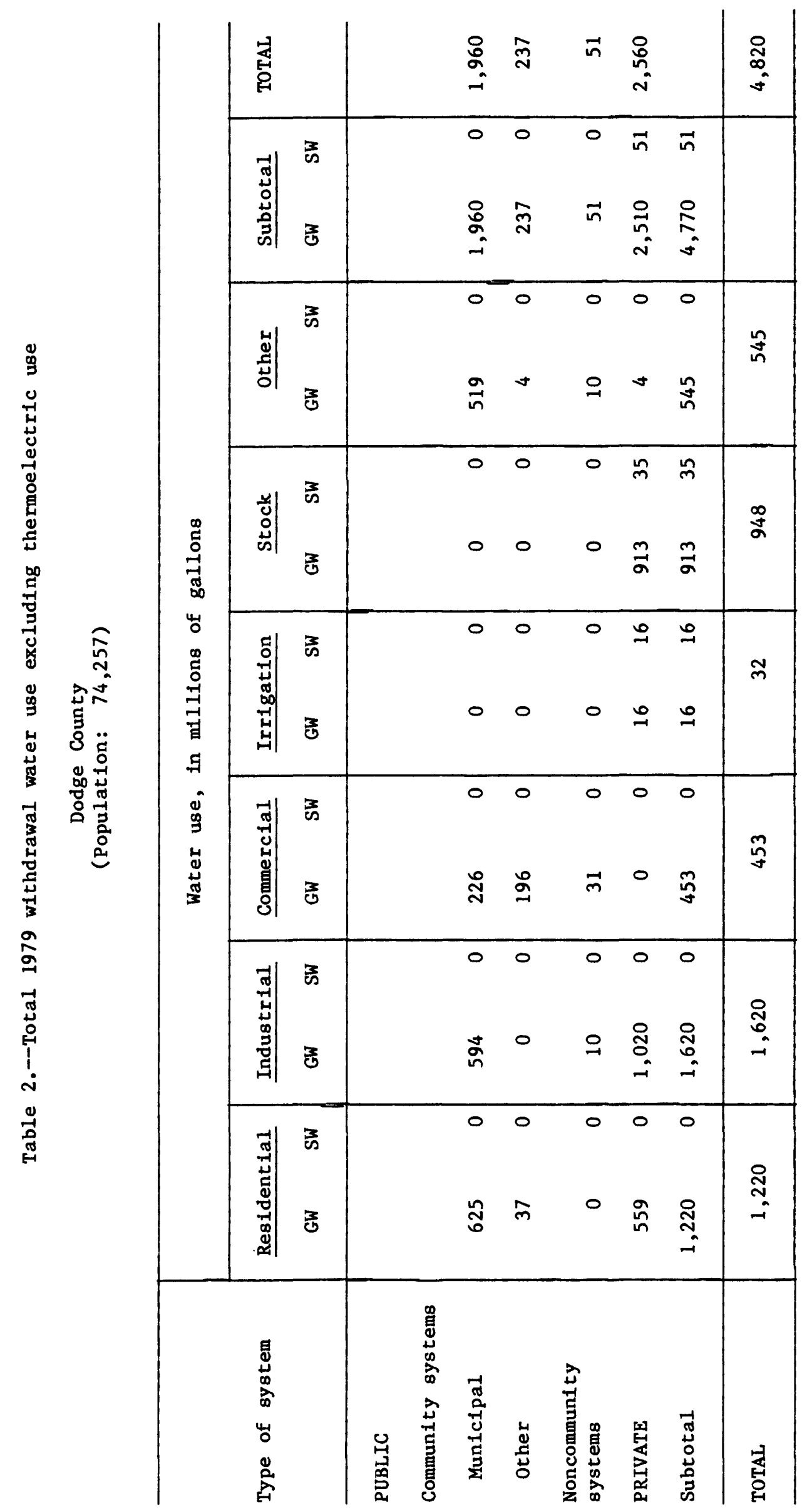




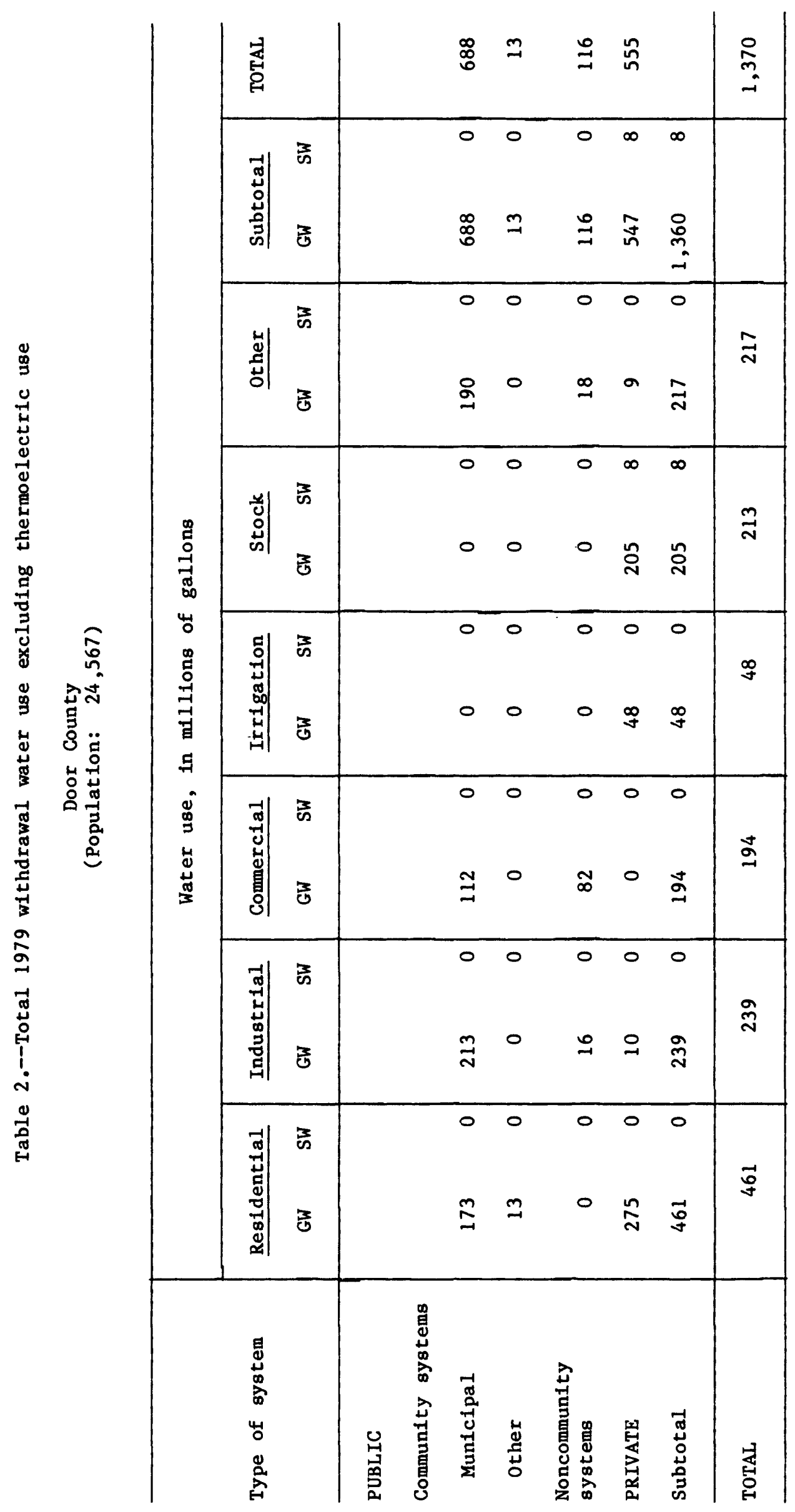




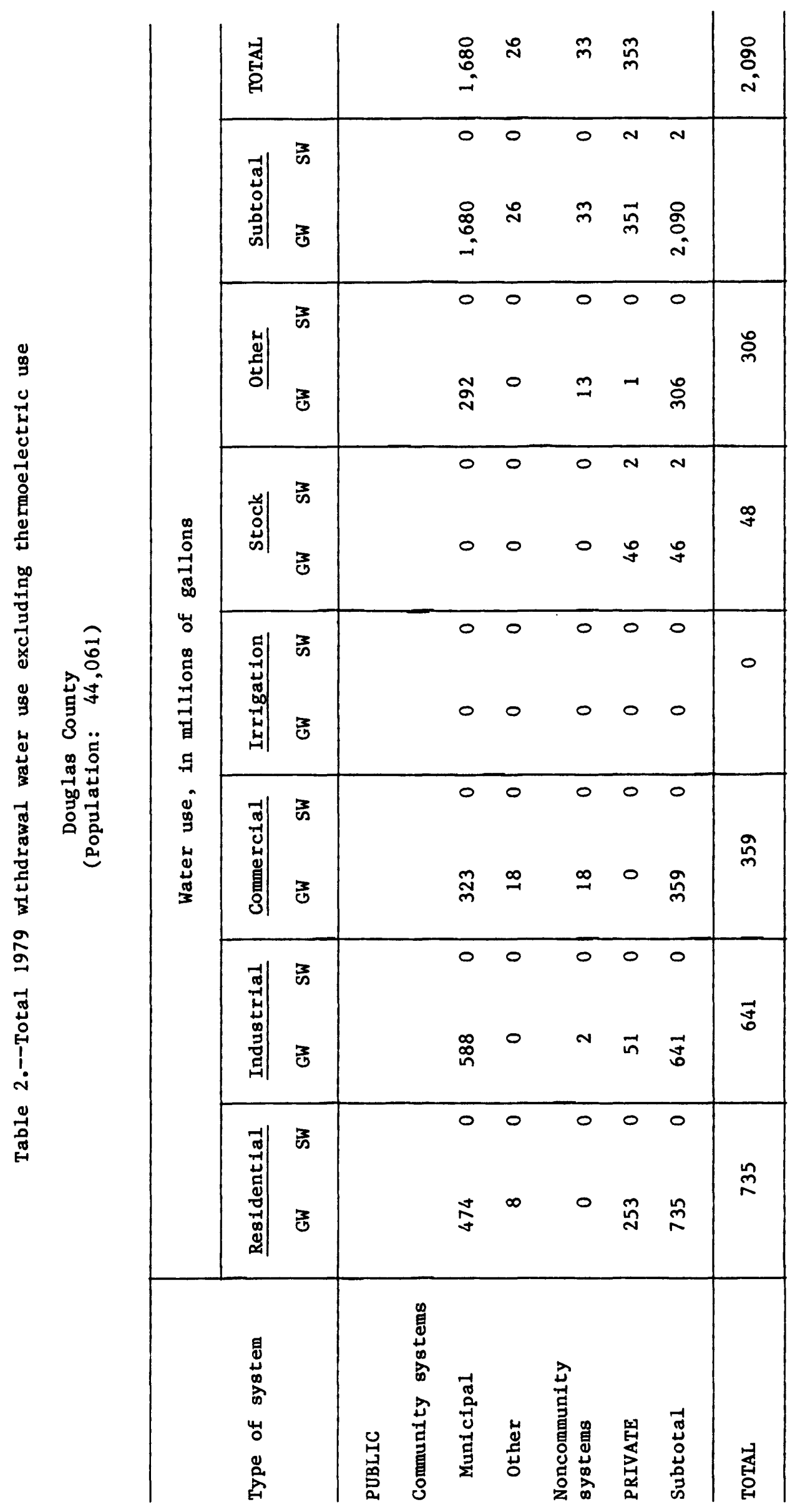




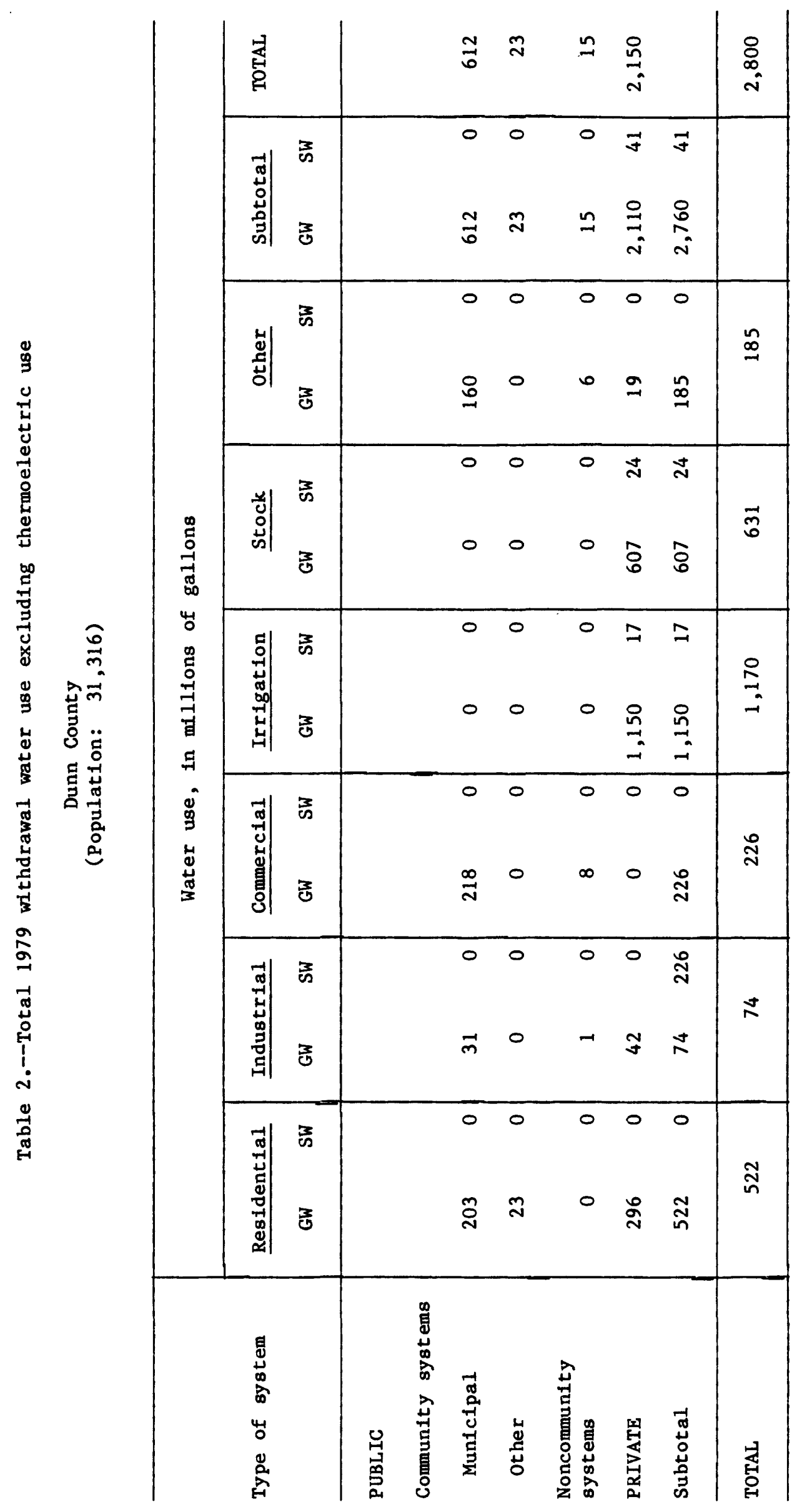




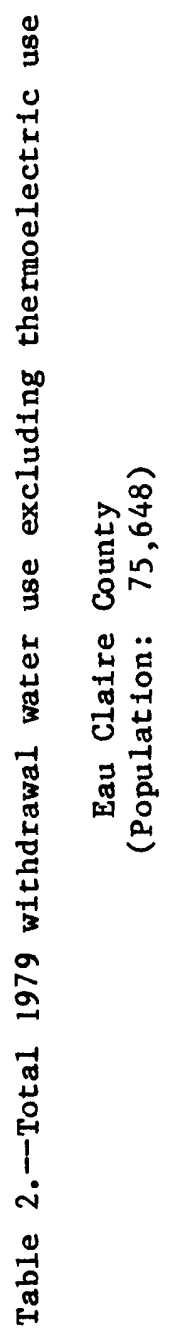

\begin{tabular}{|c|c|c|c|c|c|c|c|c|c|c|}
\hline & 是 & & & & 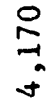 & 윰 & 吕 & $\begin{array}{l}8 \\
8 \\
0 \\
\text { in }\end{array}$ & & $\begin{array}{l}0 \\
\stackrel{0}{0} \\
\sim\end{array}$ \\
\hline & $\begin{array}{l}7 \\
0 \\
0 \\
0 \\
0 \\
0 \\
0\end{array}$ & 瓷 & & & $\begin{array}{l}0 \\
\stackrel{9}{9} \\
\stackrel{7}{0}\end{array}$ & 욱 & $\begin{array}{l}0 \\
\stackrel{N}{0}\end{array}$ & 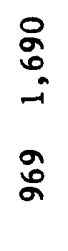 & $\begin{array}{l}8 \\
8 \\
-1 \\
-1 \\
\text { స్ } \\
\text { n. } \\
\text { n. }\end{array}$ & \\
\hline & 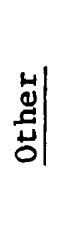 & 竕 & & & $\begin{array}{l}0 \\
\stackrel{\mathbb{N}}{0}\end{array}$ & 0 & 0 & 0 & $\begin{array}{l}0 \\
0 \\
0 \\
0 \\
0 \\
-1\end{array}$ & $\begin{array}{l}0 \\
0 \\
0 \\
\text { - }\end{array}$ \\
\hline 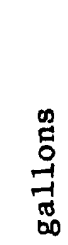 & $\begin{array}{l}\text { Uै। } \\
\text { मे }\end{array}$ & 疍 & & & $\begin{array}{l}0 \\
0\end{array}$ & $\begin{array}{l}0 \\
0\end{array}$ & $\begin{array}{l}0 \\
0\end{array}$ & $\begin{array}{l}\cong \\
\stackrel{\sim}{m}\end{array}$ & $\begin{array}{l}\approx \\
\underset{m}{\sim}\end{array}$ & $\underset{\sim}{\sim}$ \\
\hline 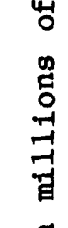 & 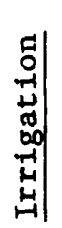 & $\begin{array}{l}3 \\
3 \\
3\end{array}$ & & & 0 & 0 & $\begin{array}{l}0 \\
0\end{array}$ & $\begin{array}{l}\text { n } \\
\text { సิ }\end{array}$ & $\begin{array}{l}\text { ก } \\
\text { స్ }\end{array}$ & 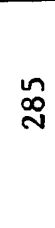 \\
\hline 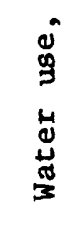 & $\begin{array}{l}-1 \\
0 \\
0 \\
\vdots \\
0 \\
⿱ 10 \\
0\end{array}$ & $\begin{array}{l}3 \\
3 \\
3\end{array}$ & & & $\begin{array}{l}0 \\
\text { o }\end{array}$ & 0 & $\begin{array}{l}0 \\
\pm\end{array}$ & $\begin{array}{l}0 \\
0\end{array}$ & $\begin{array}{l}0 \\
\text { nn }\end{array}$ & in \\
\hline & 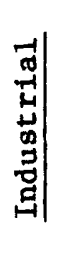 & 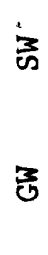 & & & $\begin{array}{l}0 \\
0 \\
\text { ㅇ. } \\
0 . \\
-1\end{array}$ & $\begin{array}{l}0 \\
0\end{array}$ & $\begin{array}{l}0 \\
\text { in }\end{array}$ & 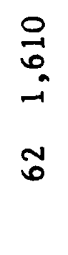 & $\begin{array}{l}0 \\
0 \\
0 \\
-1 \\
8 \\
8 \\
0 \\
-1\end{array}$ & $\begin{array}{l}\text { 尺े } \\
\text { m. }\end{array}$ \\
\hline & 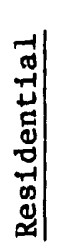 & 录 & & & $\begin{array}{l}0 \\
\stackrel{0}{*} \\
-1\end{array}$ & 0 & $\begin{array}{l}0 \\
0\end{array}$ & $\begin{array}{l}0 \\
\stackrel{m}{\sim}\end{array}$ & $\begin{array}{l}0 \\
0 \\
0 \\
0 \\
0 \\
-1\end{array}$ & $\begin{array}{l}8 \\
8 \\
+ \\
-1\end{array}$ \\
\hline & 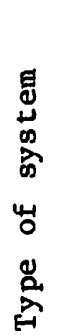 & & 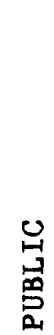 & 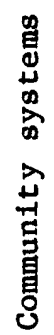 & 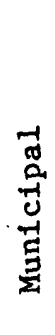 & $\frac{\breve{d}}{\Delta}$ & 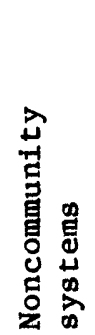 & 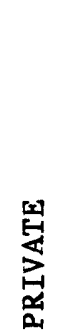 & 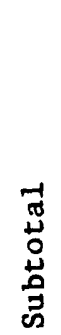 & 趈 \\
\hline
\end{tabular}




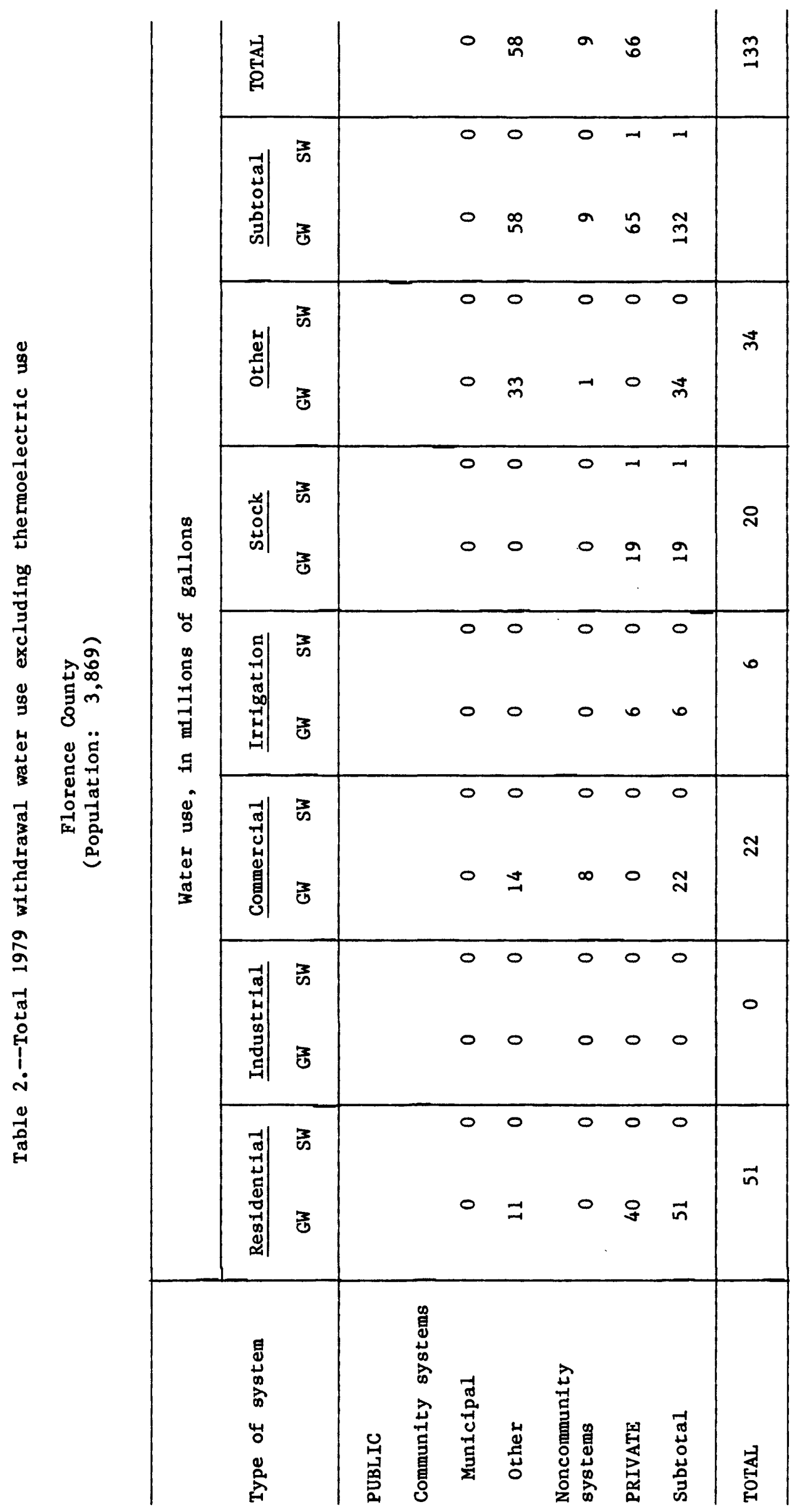




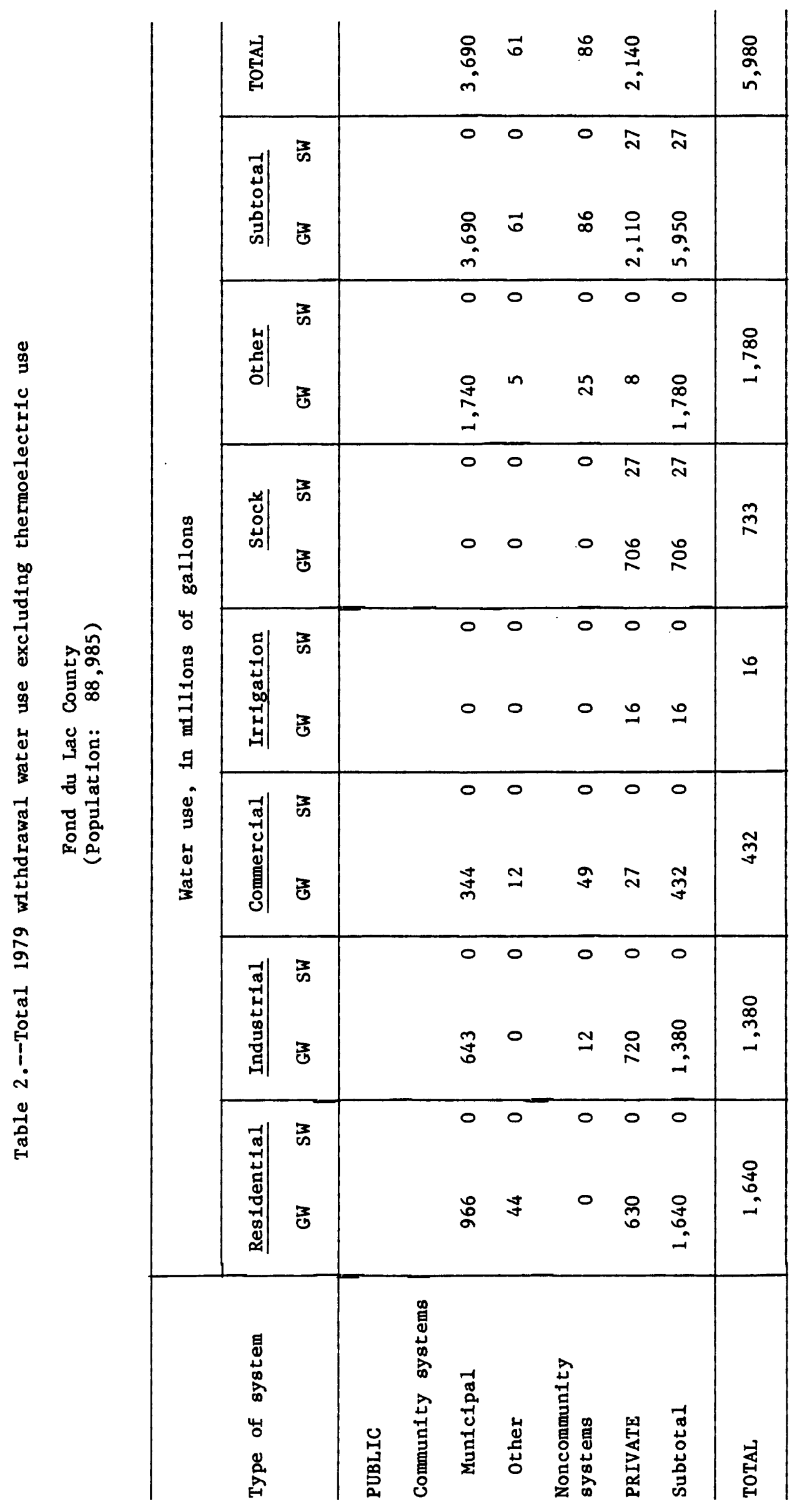




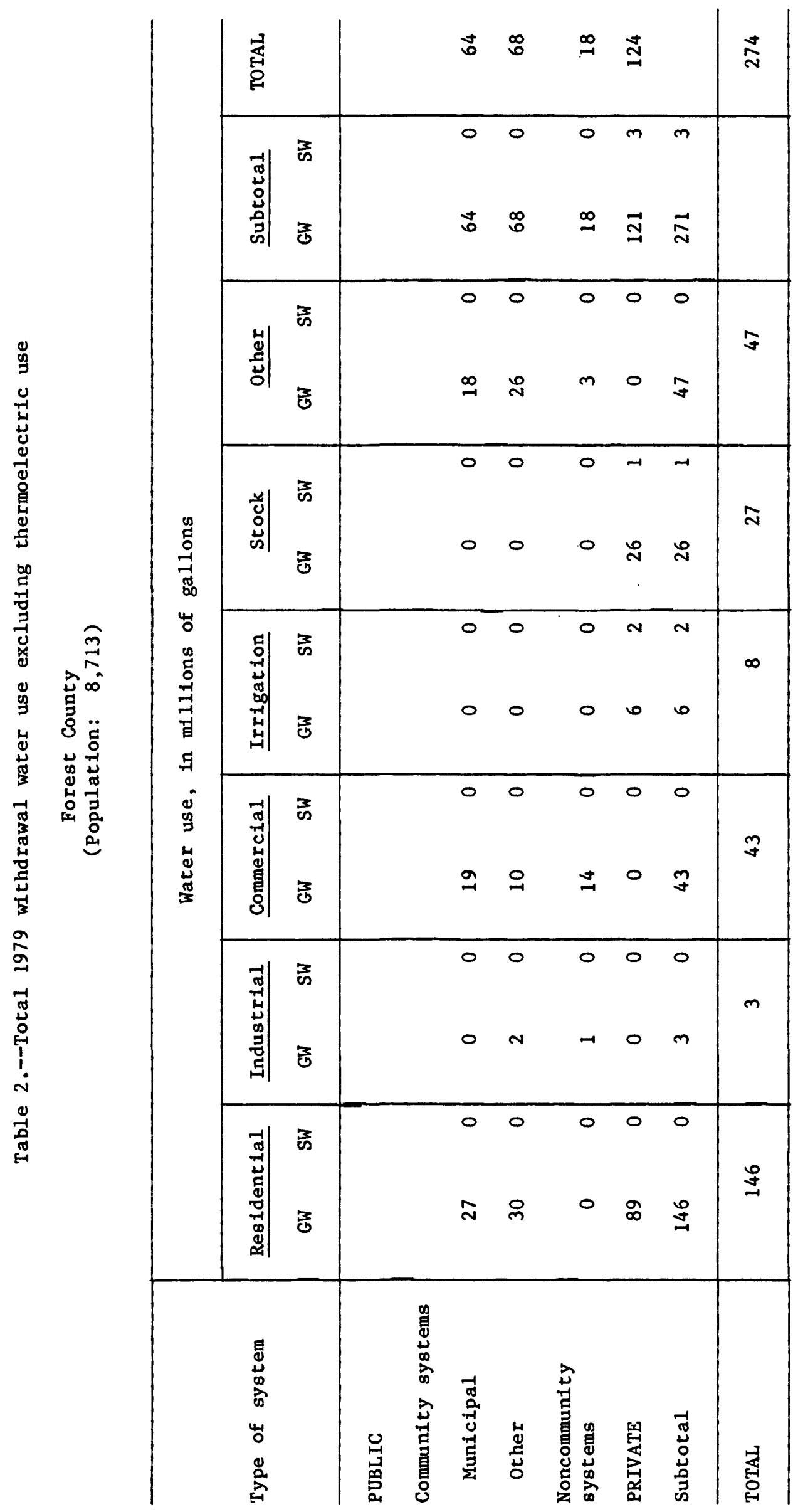




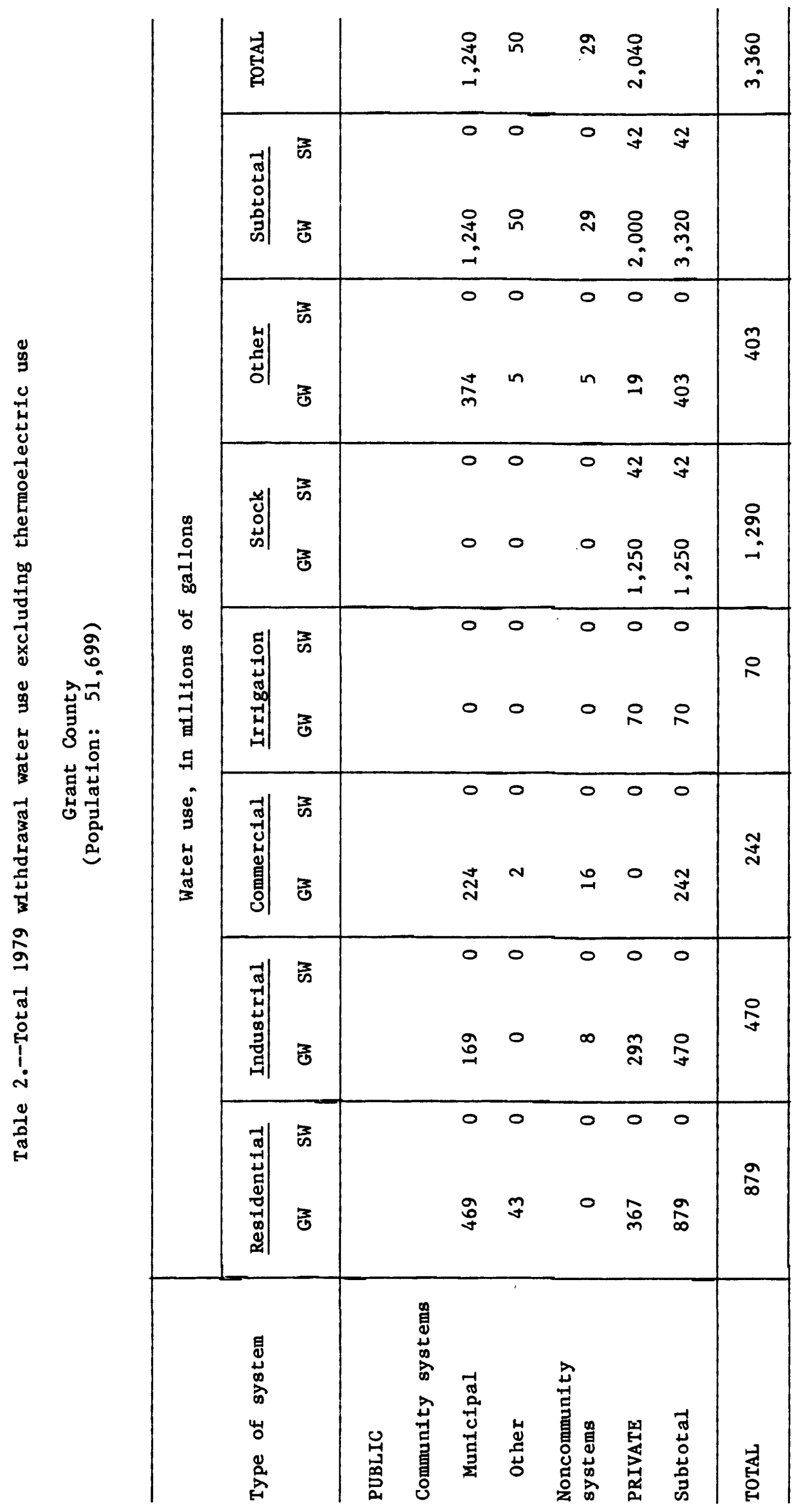




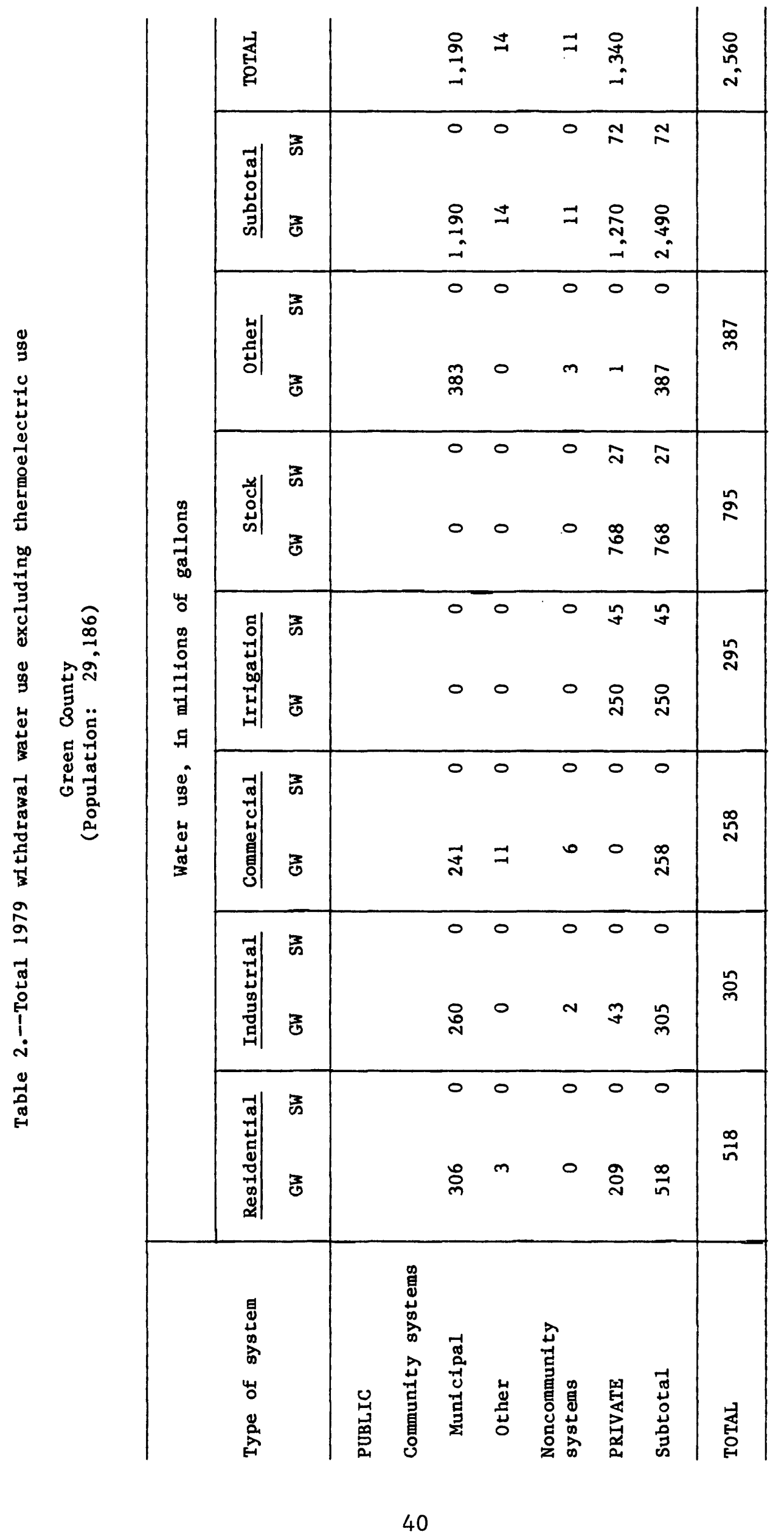




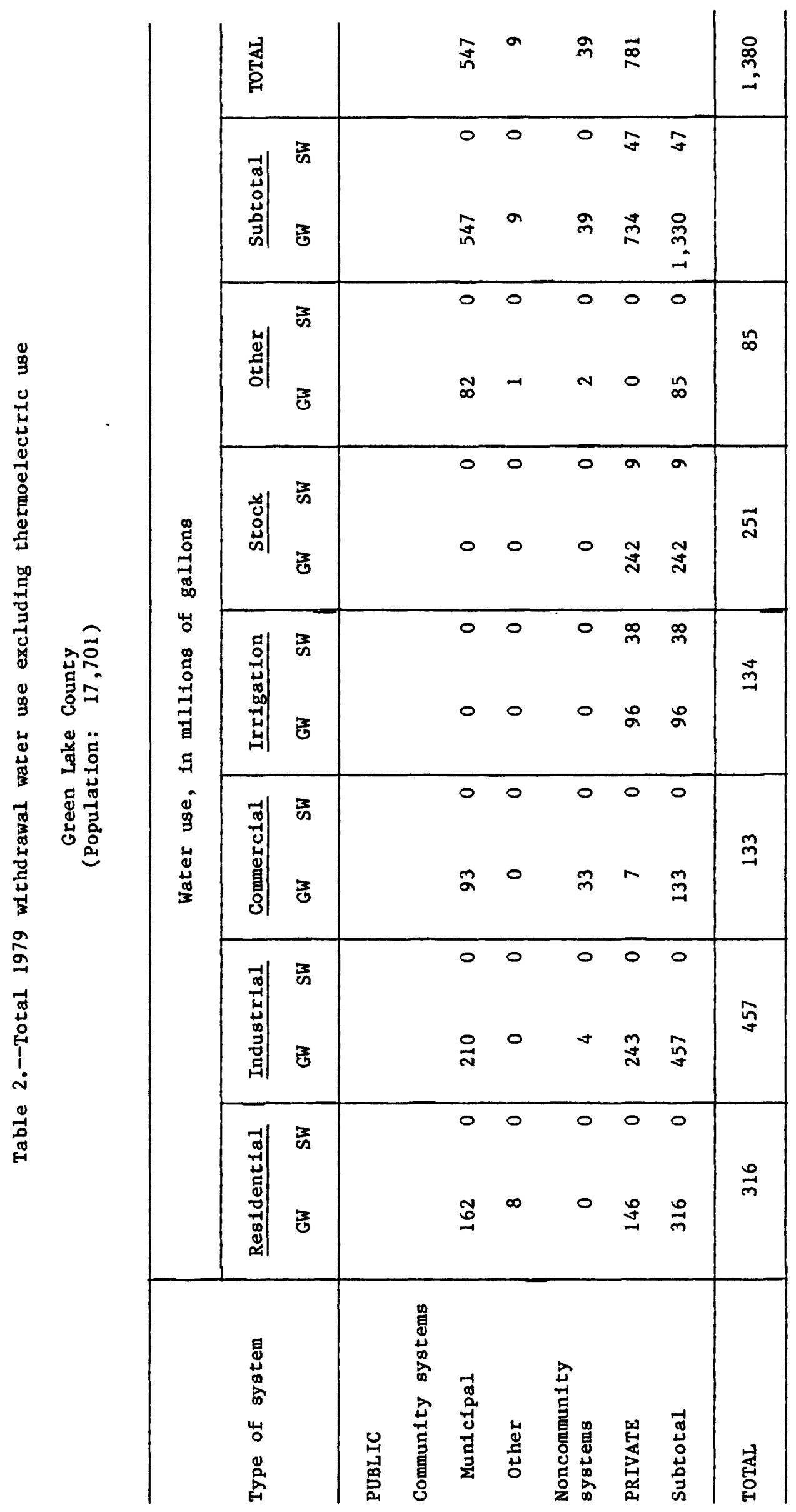




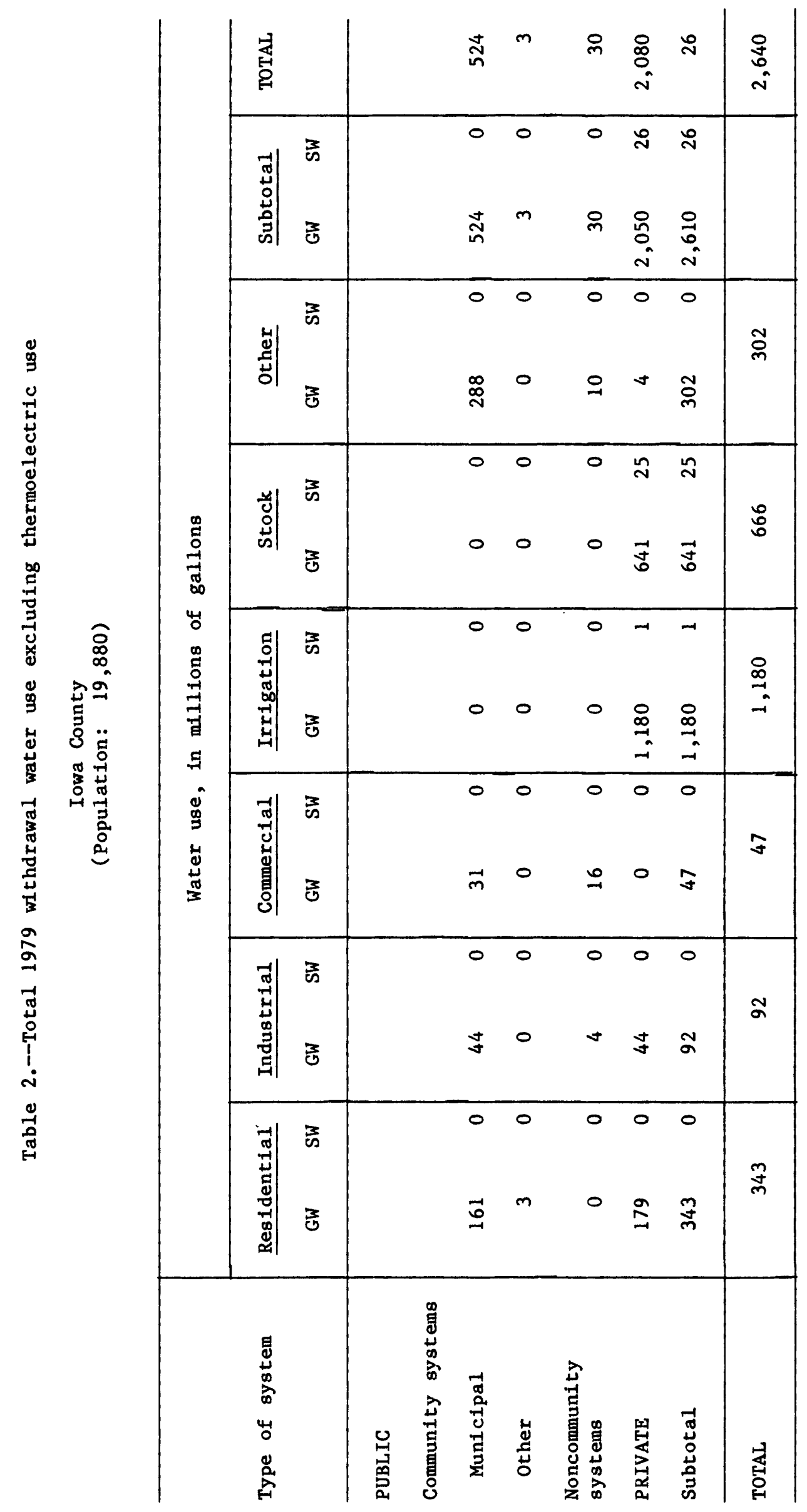




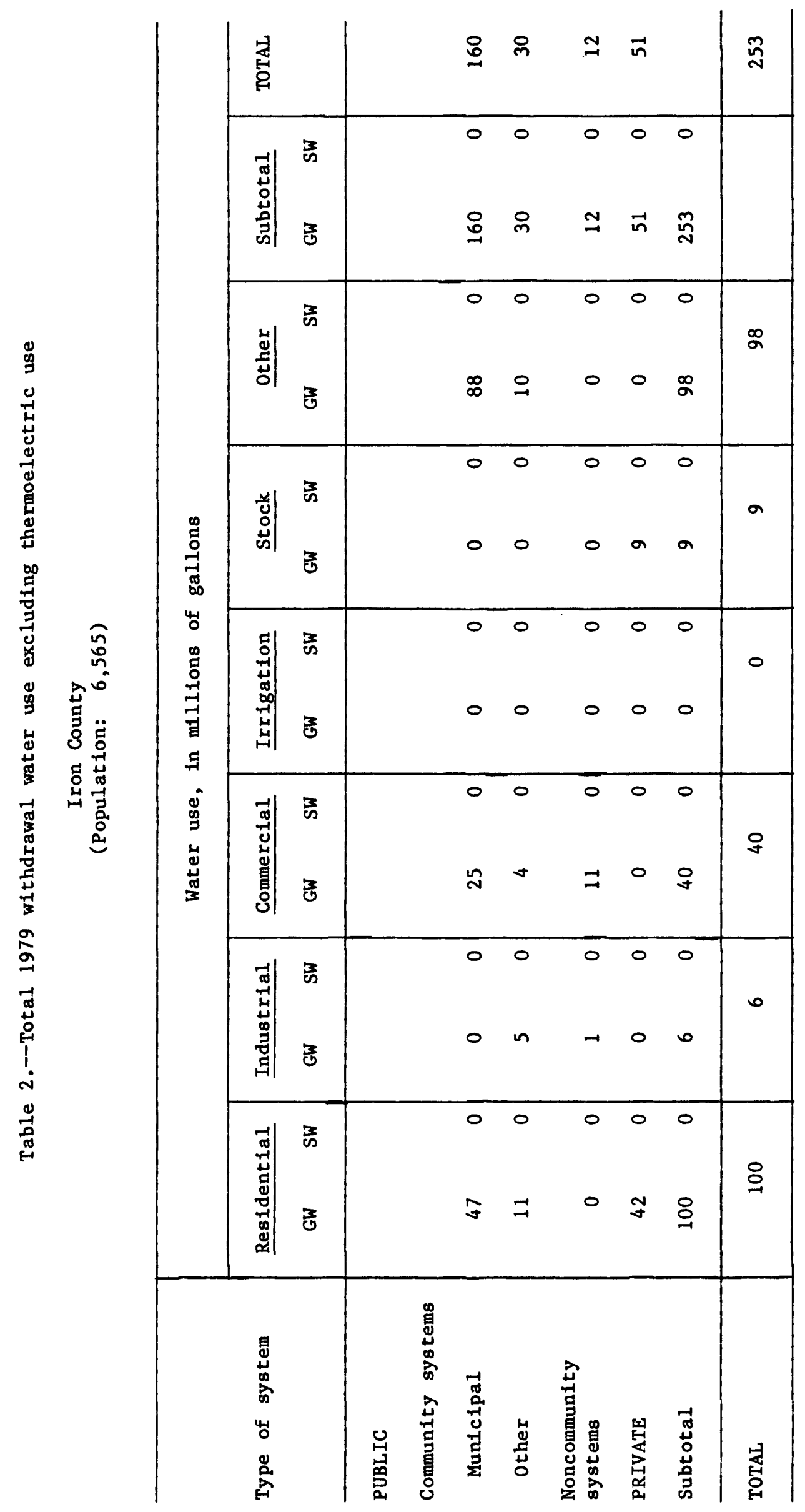




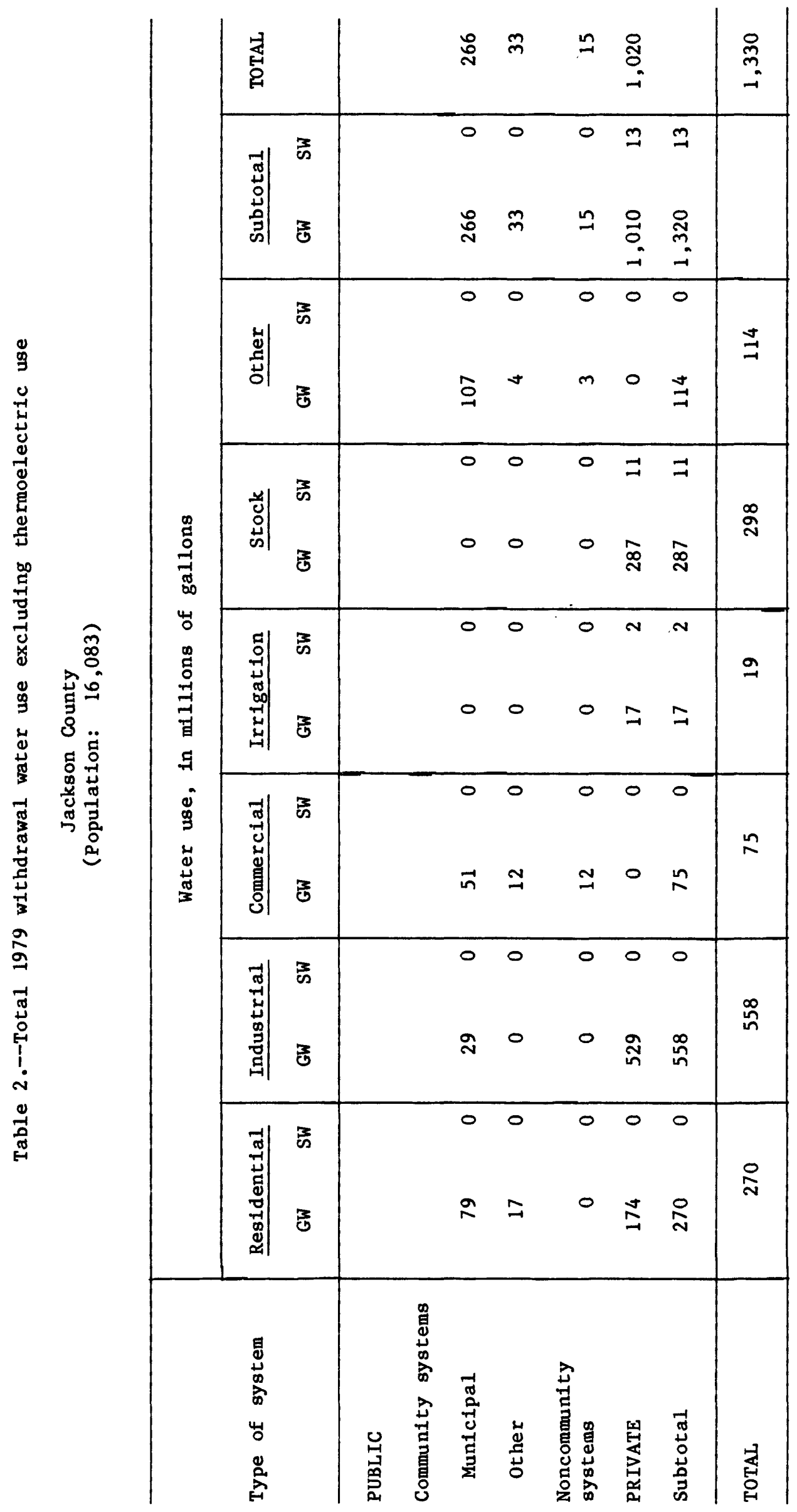




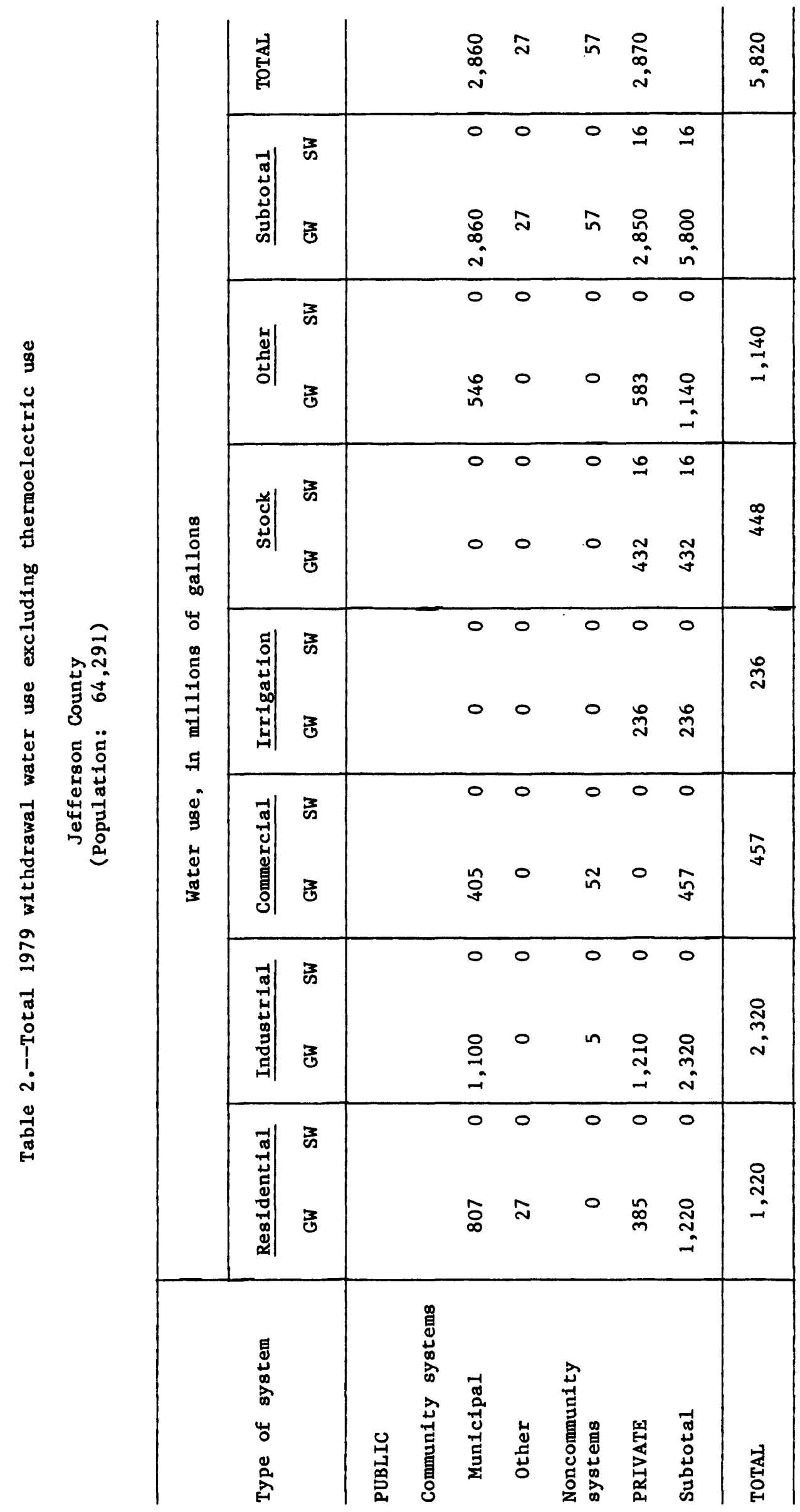




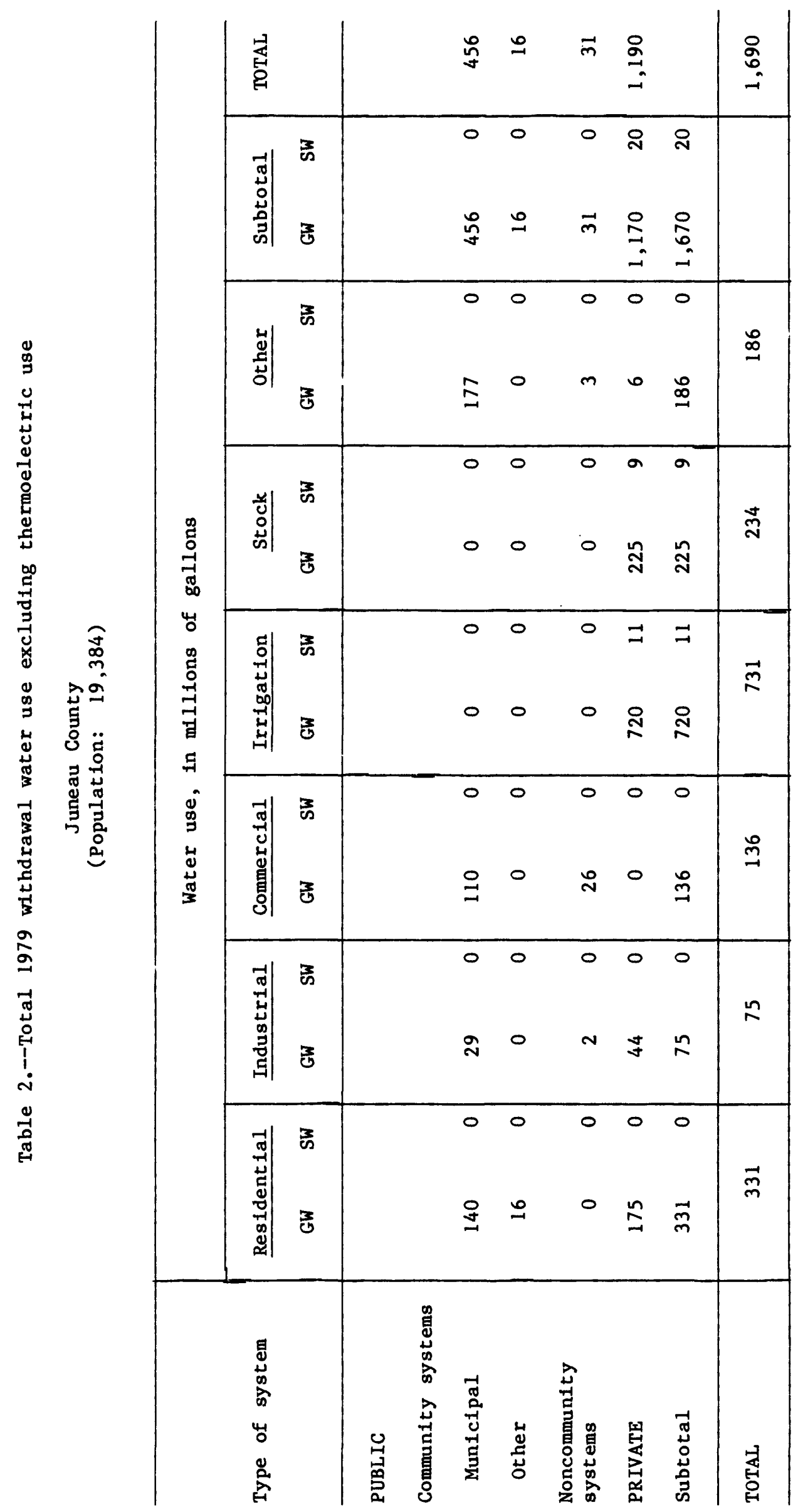




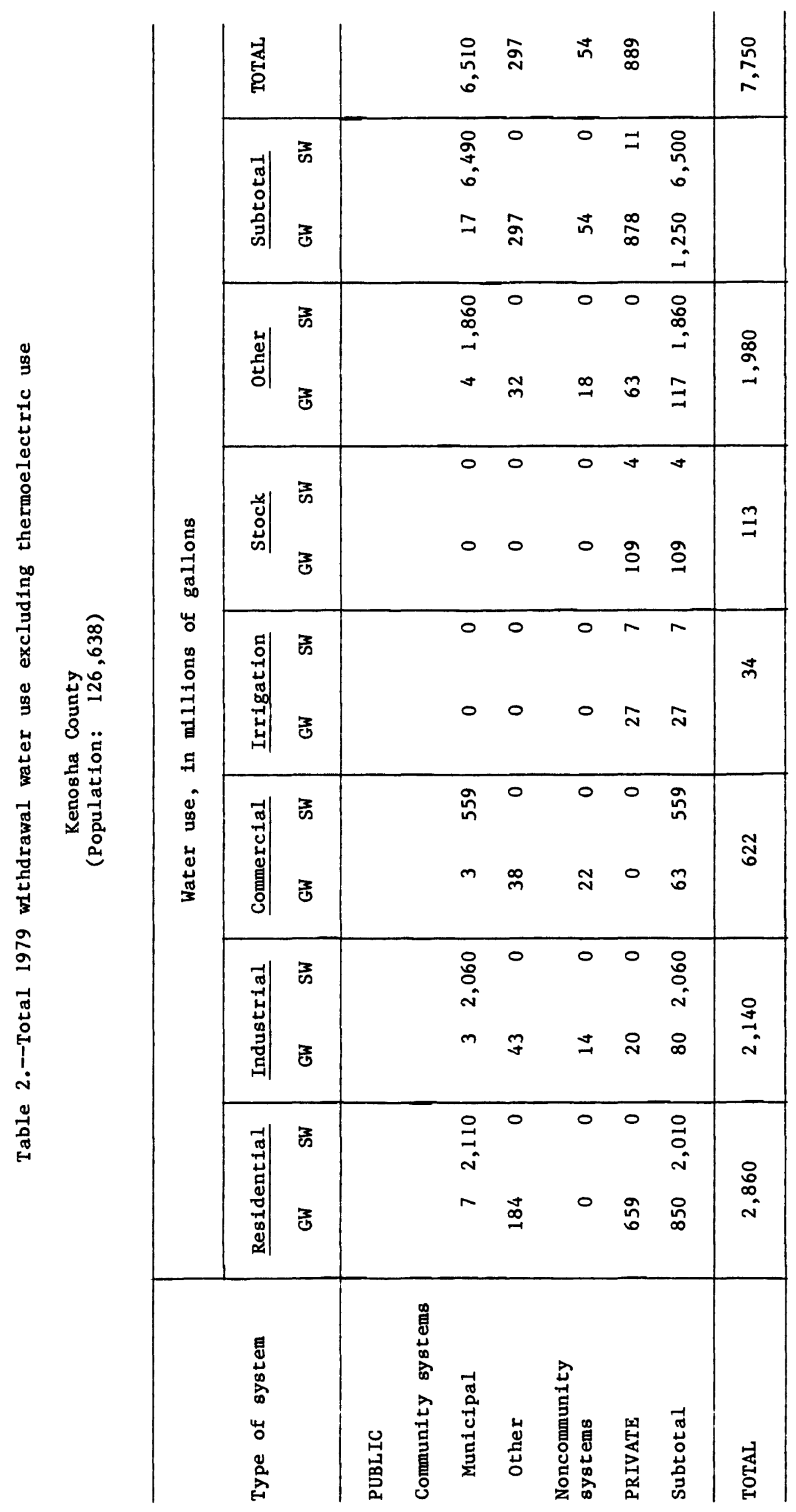




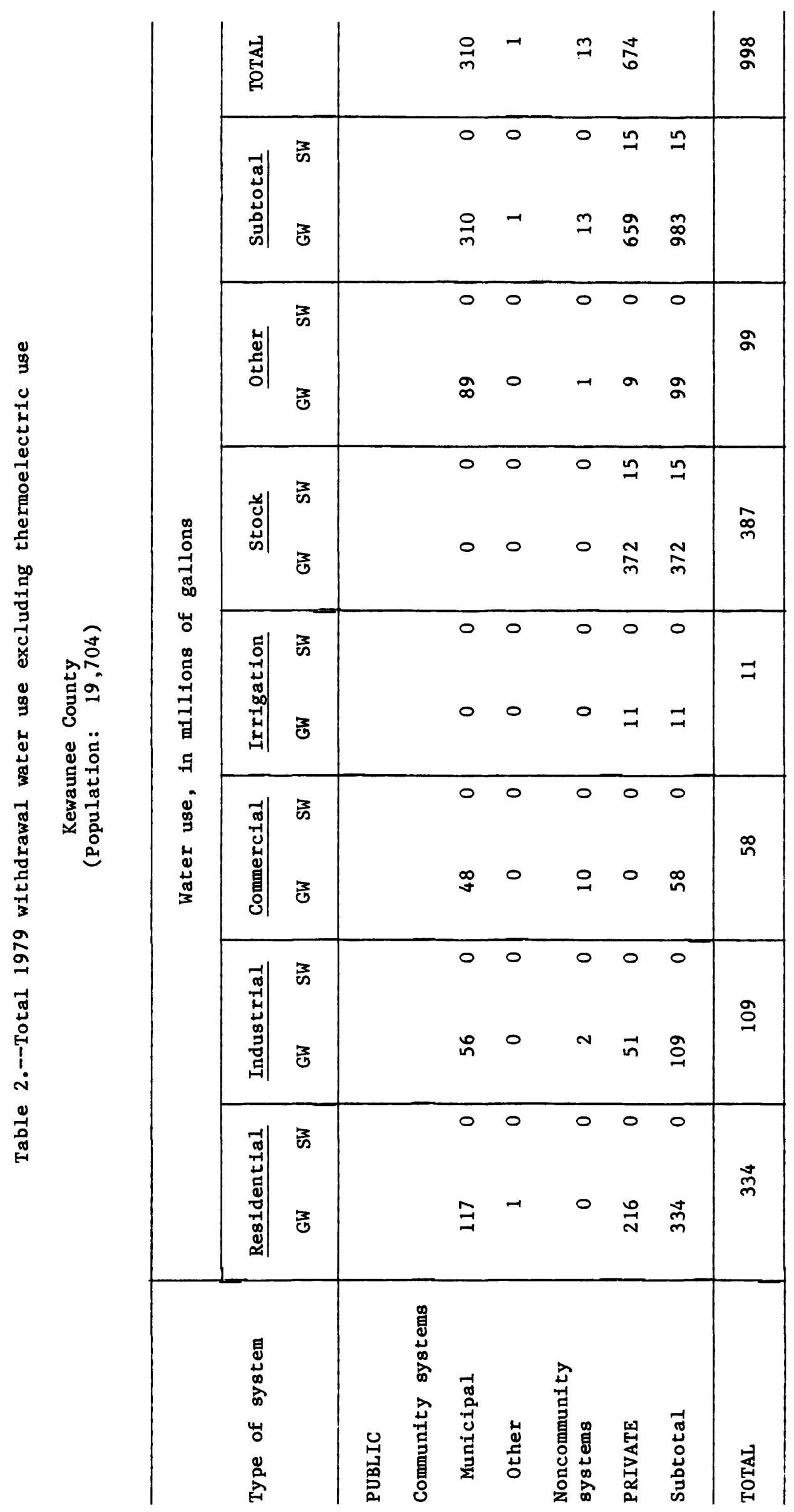




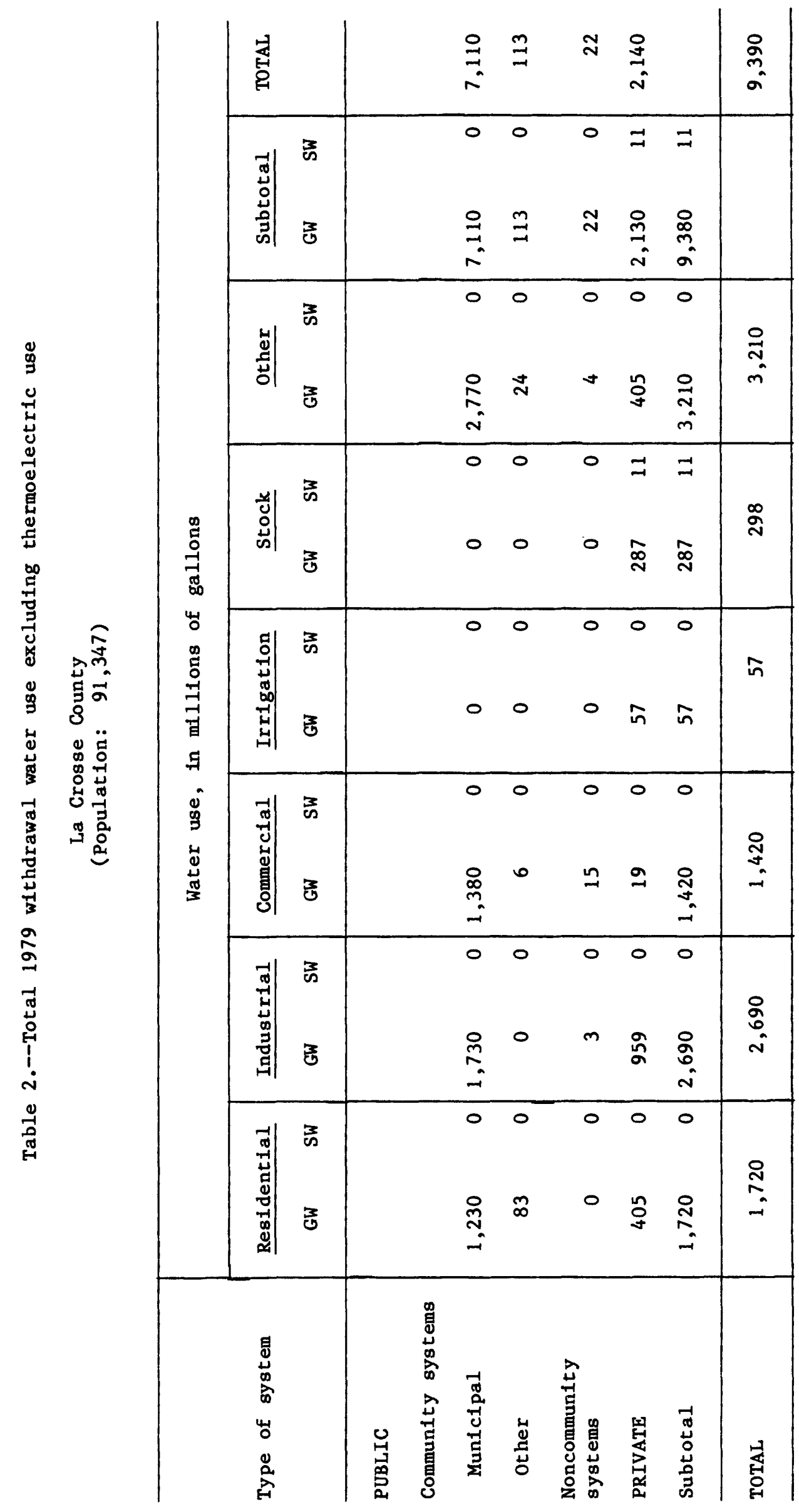




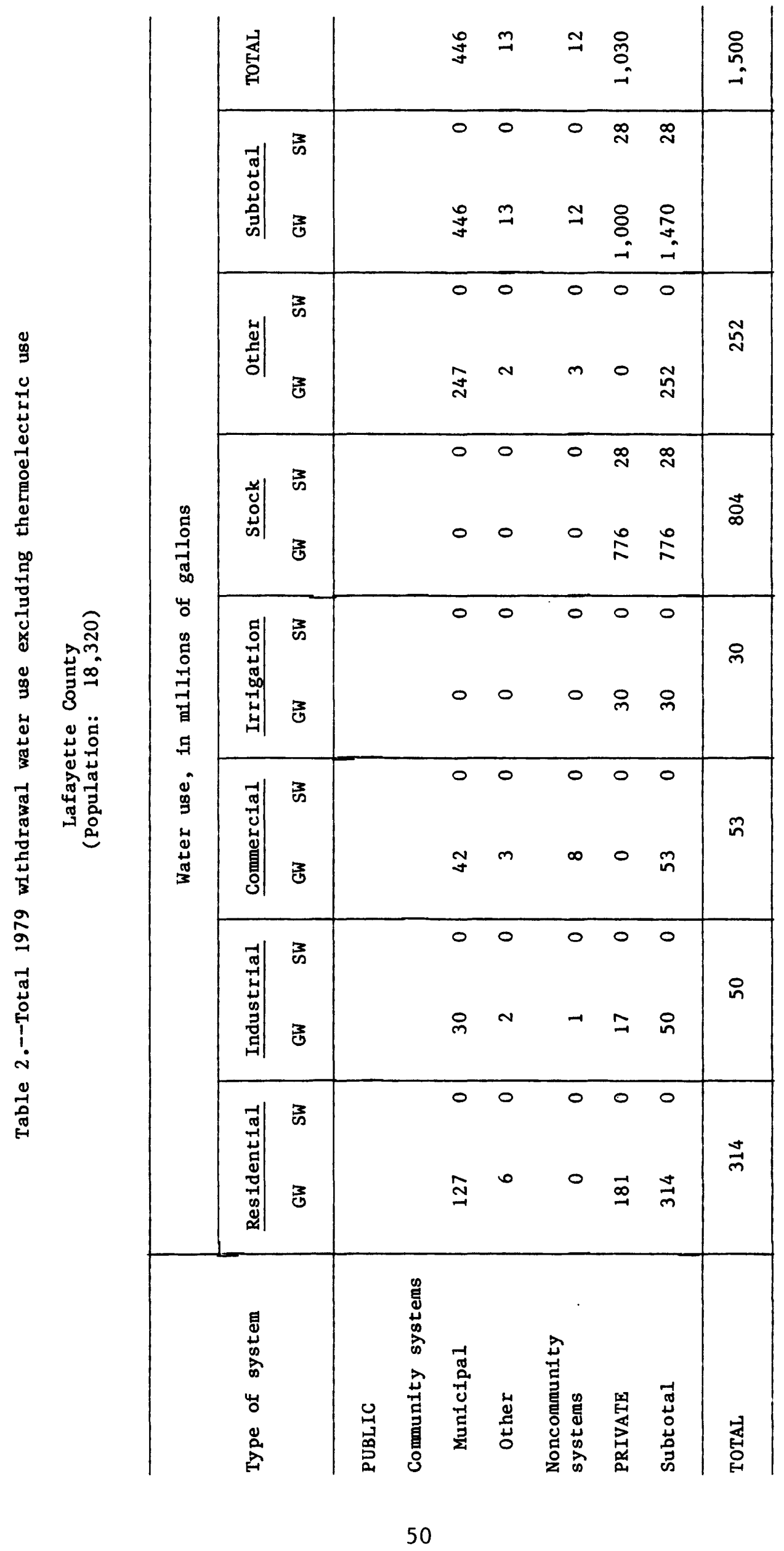




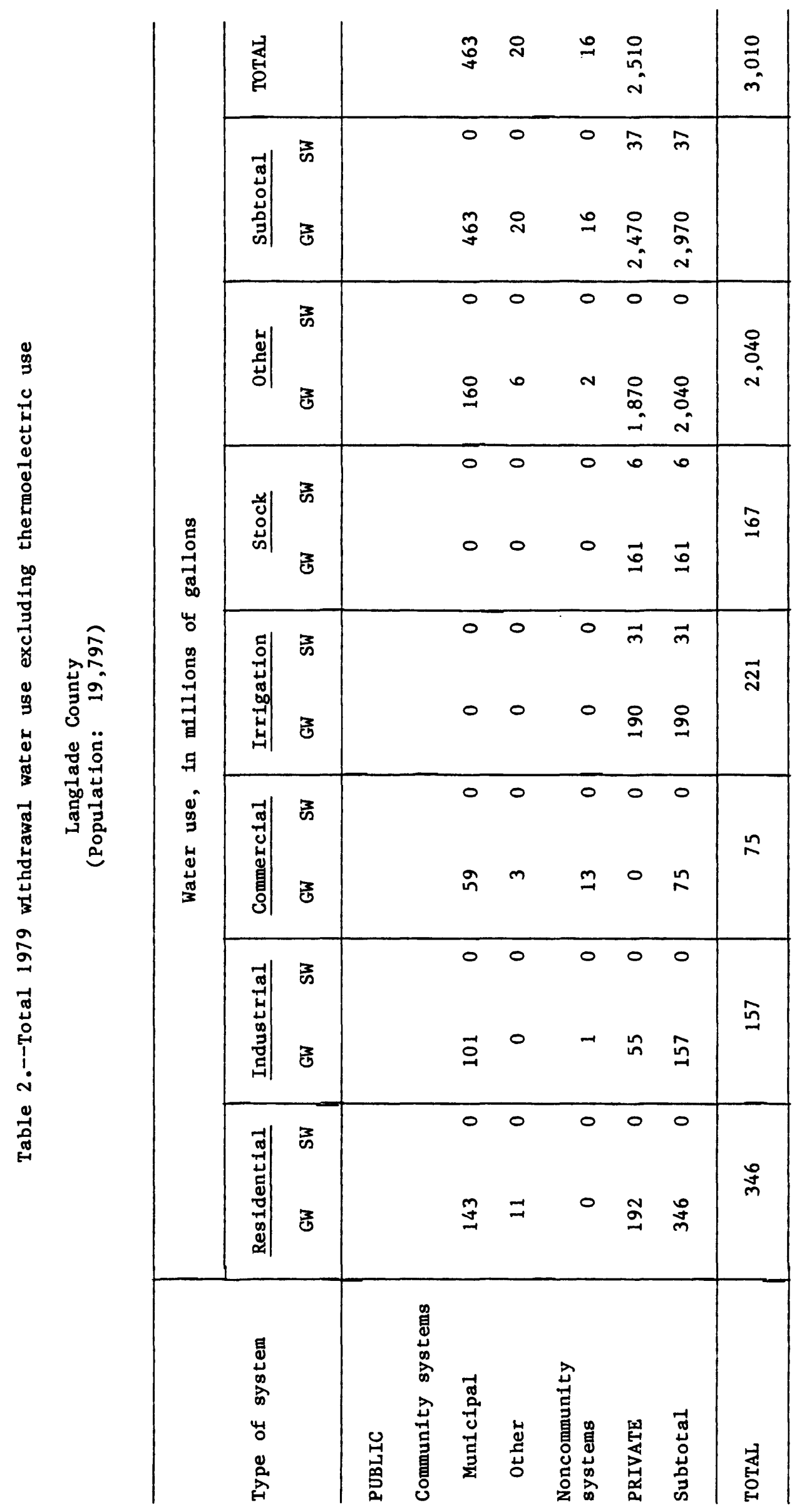




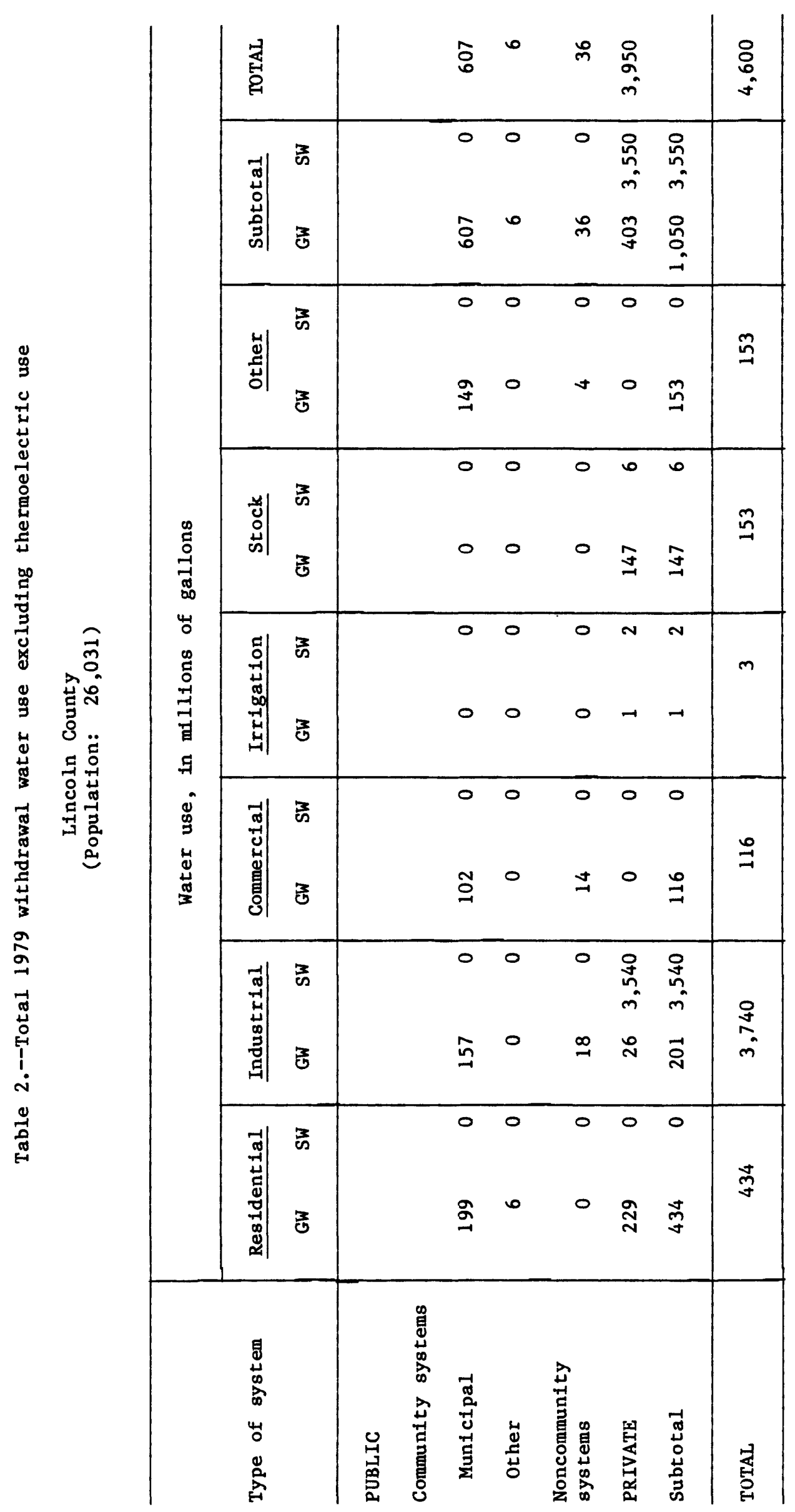




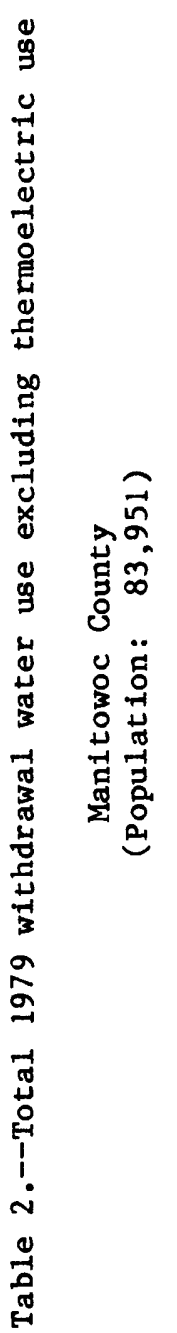

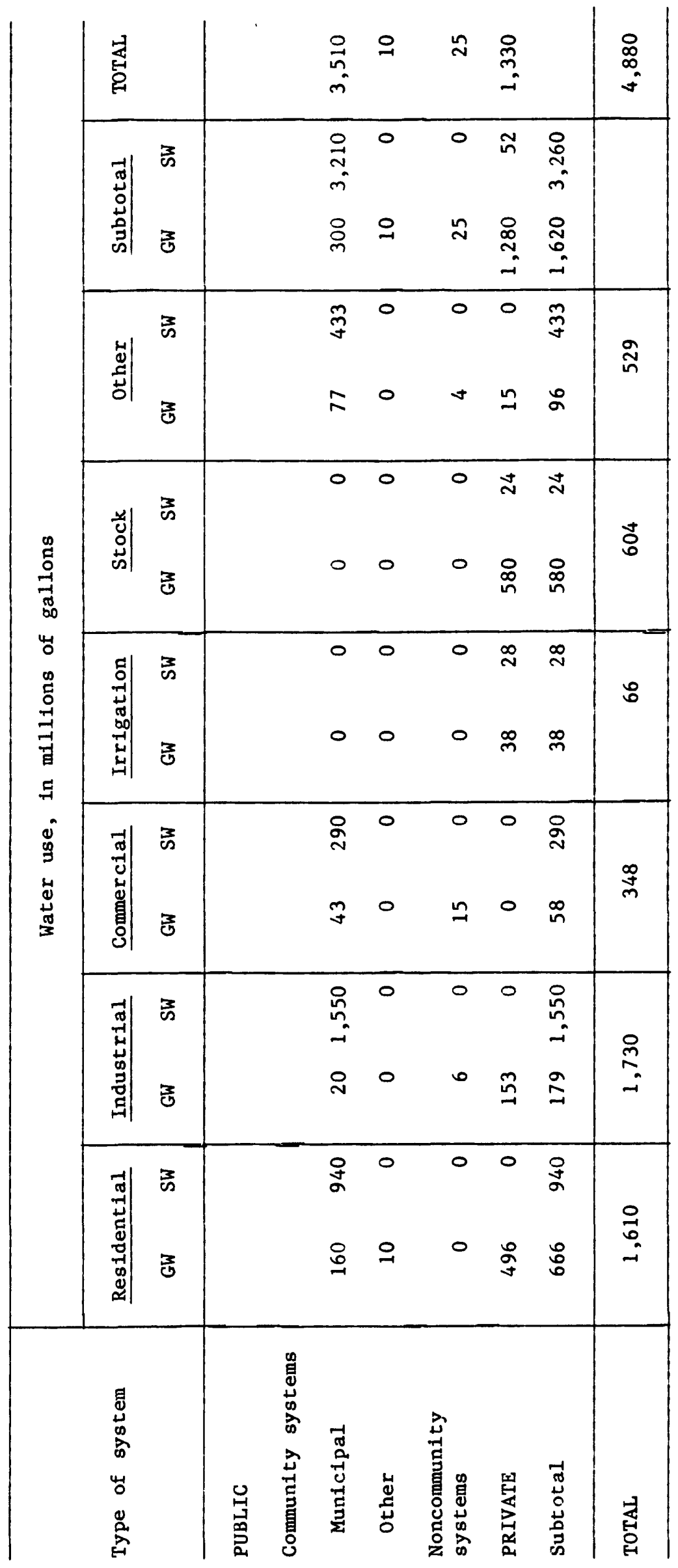




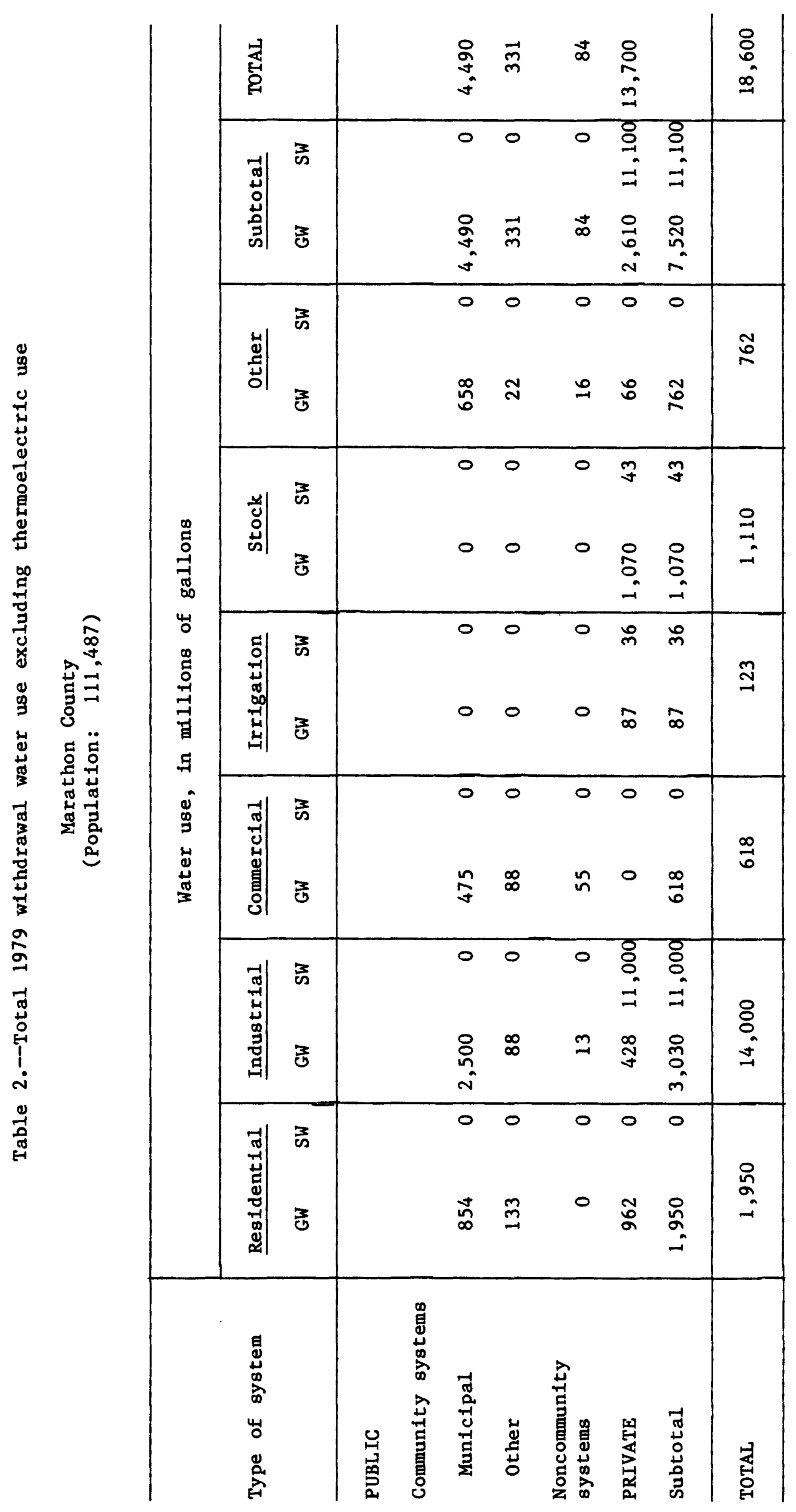




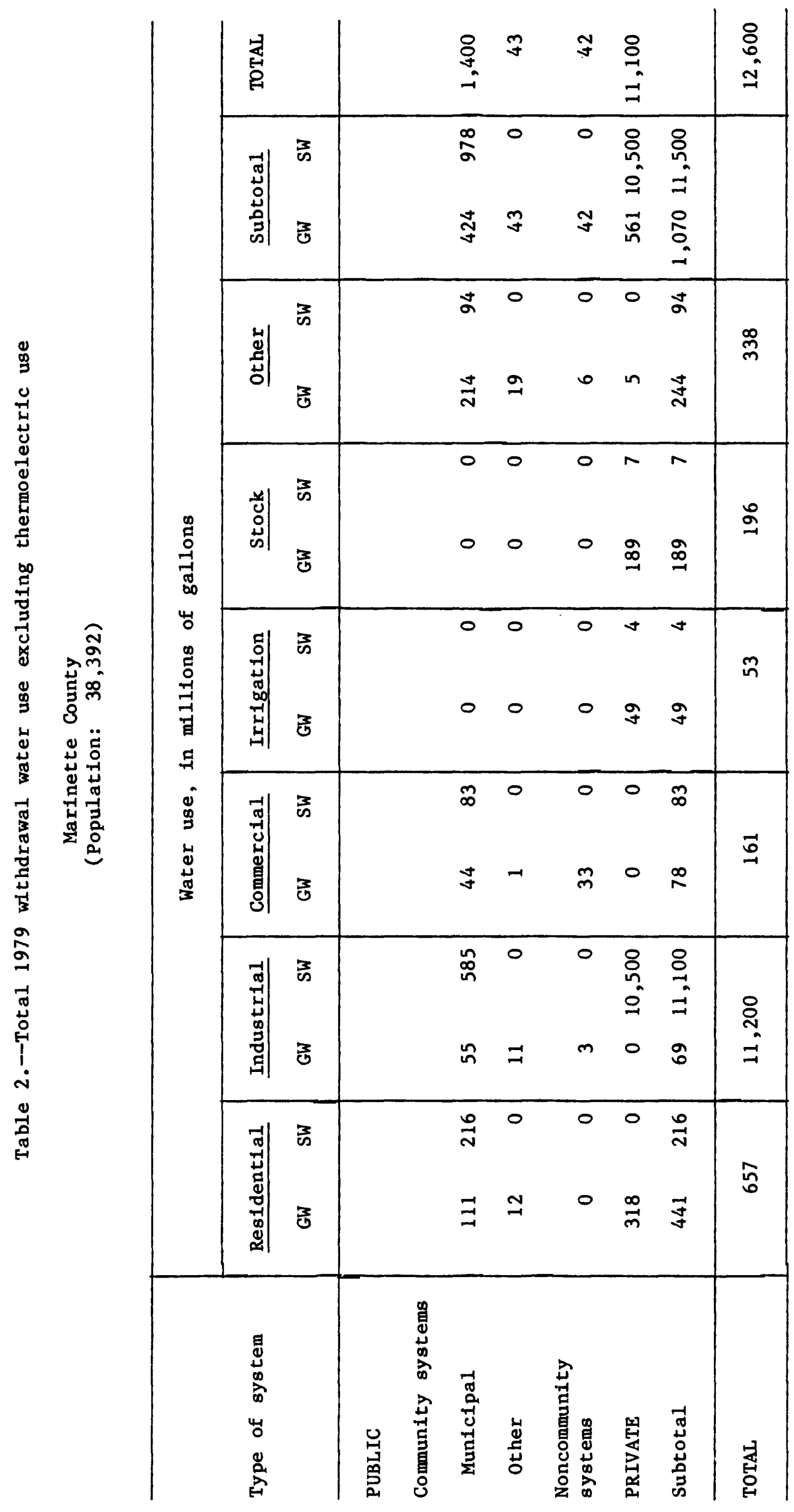




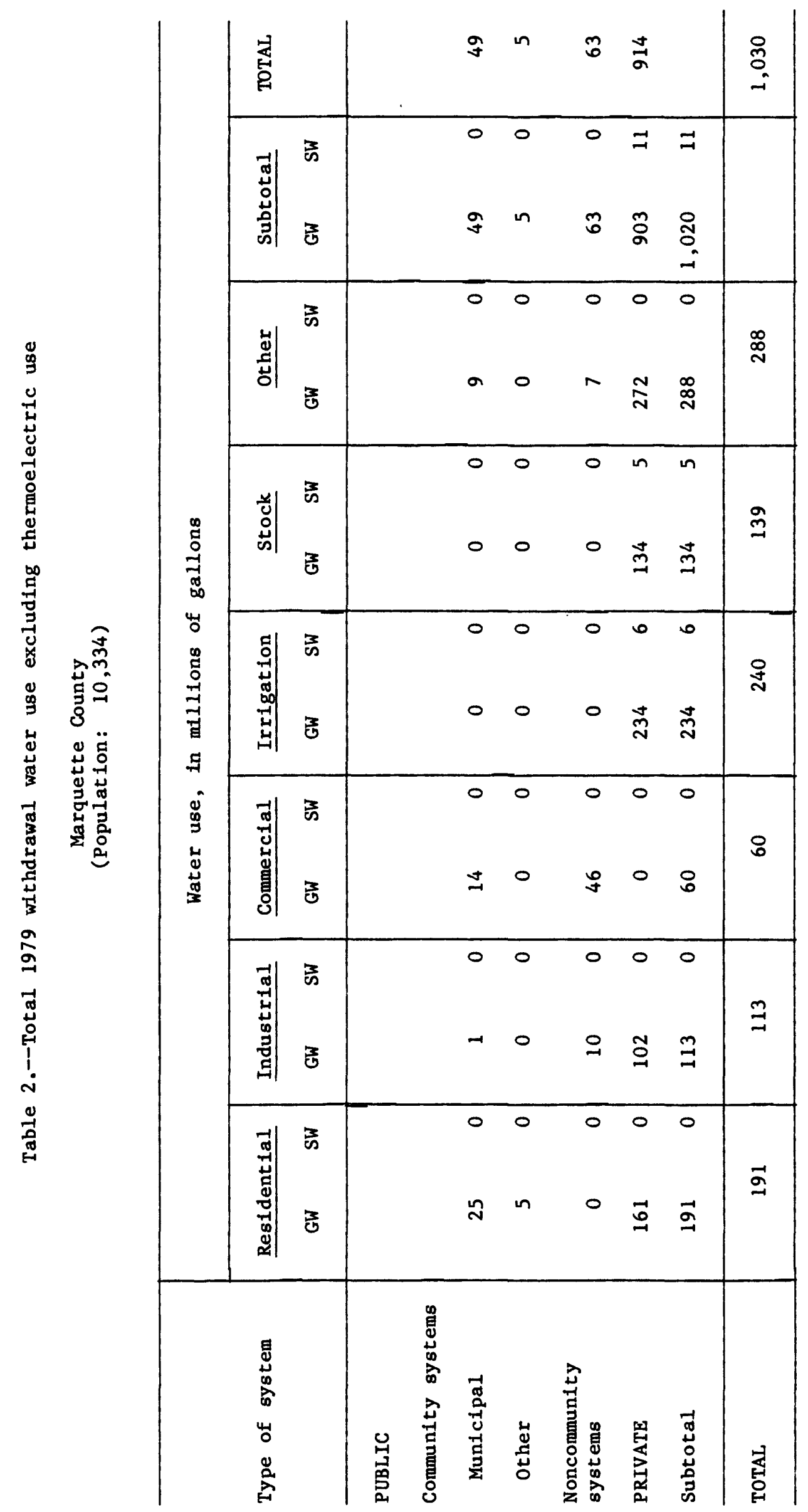




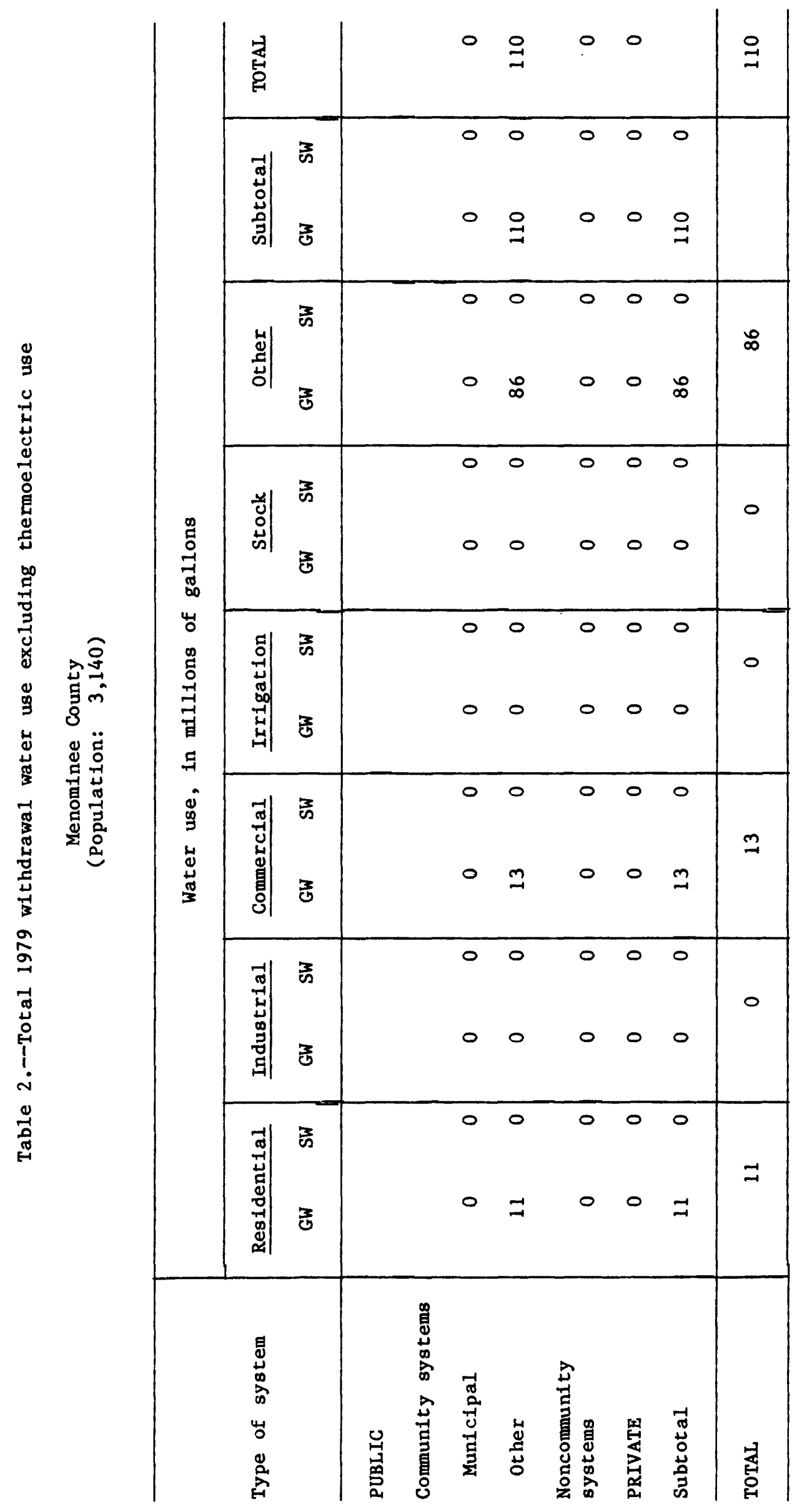




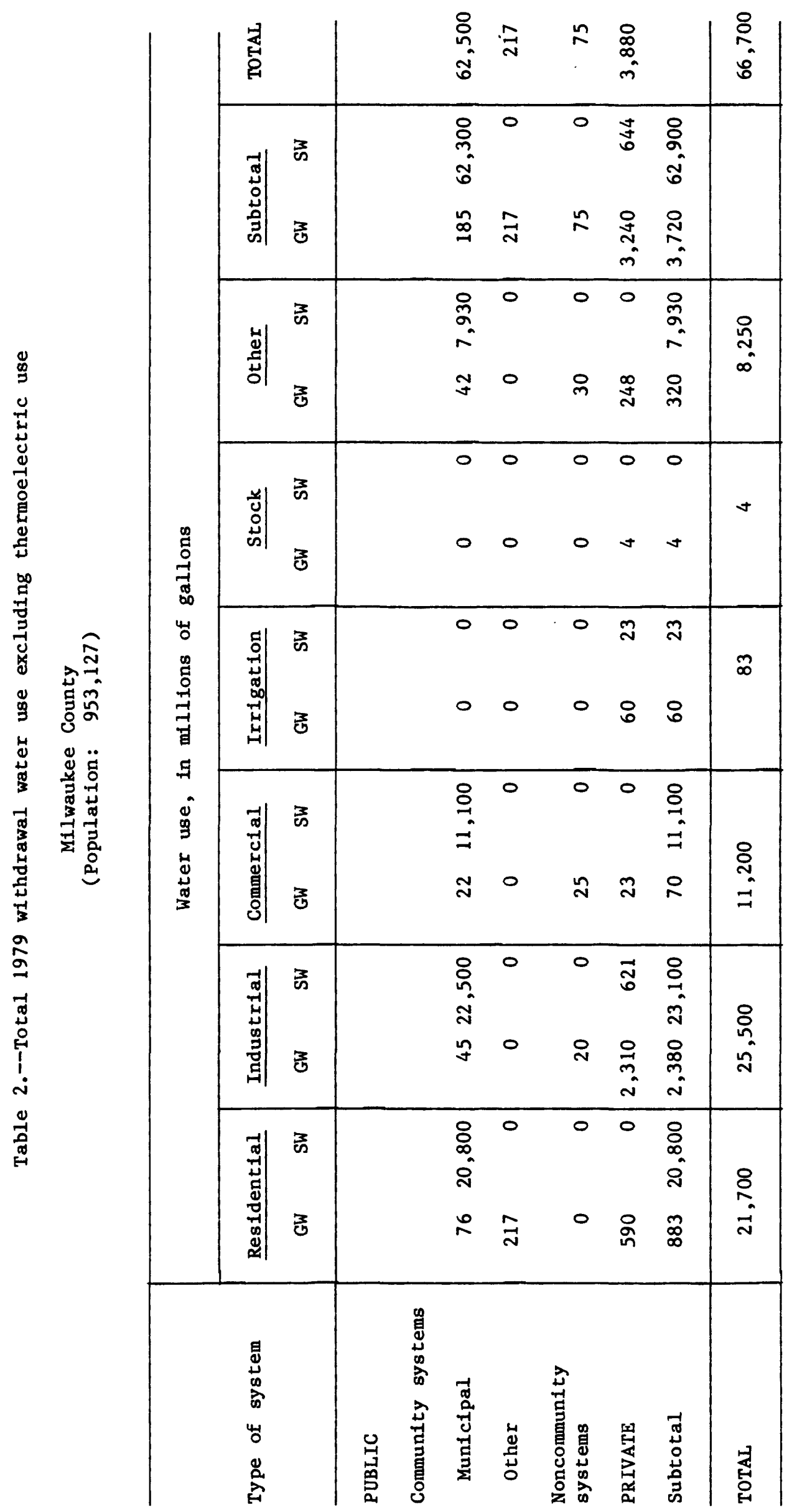




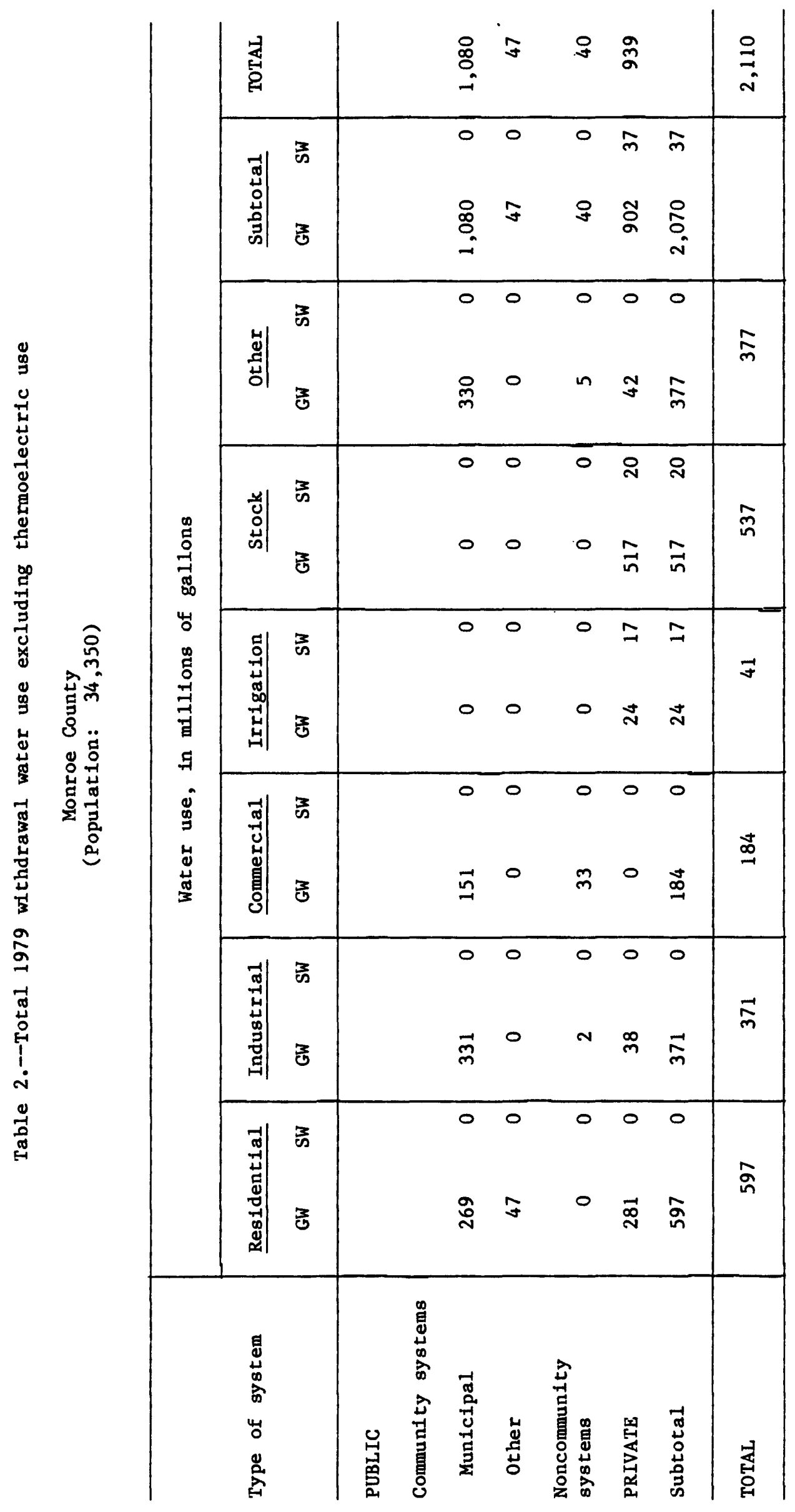




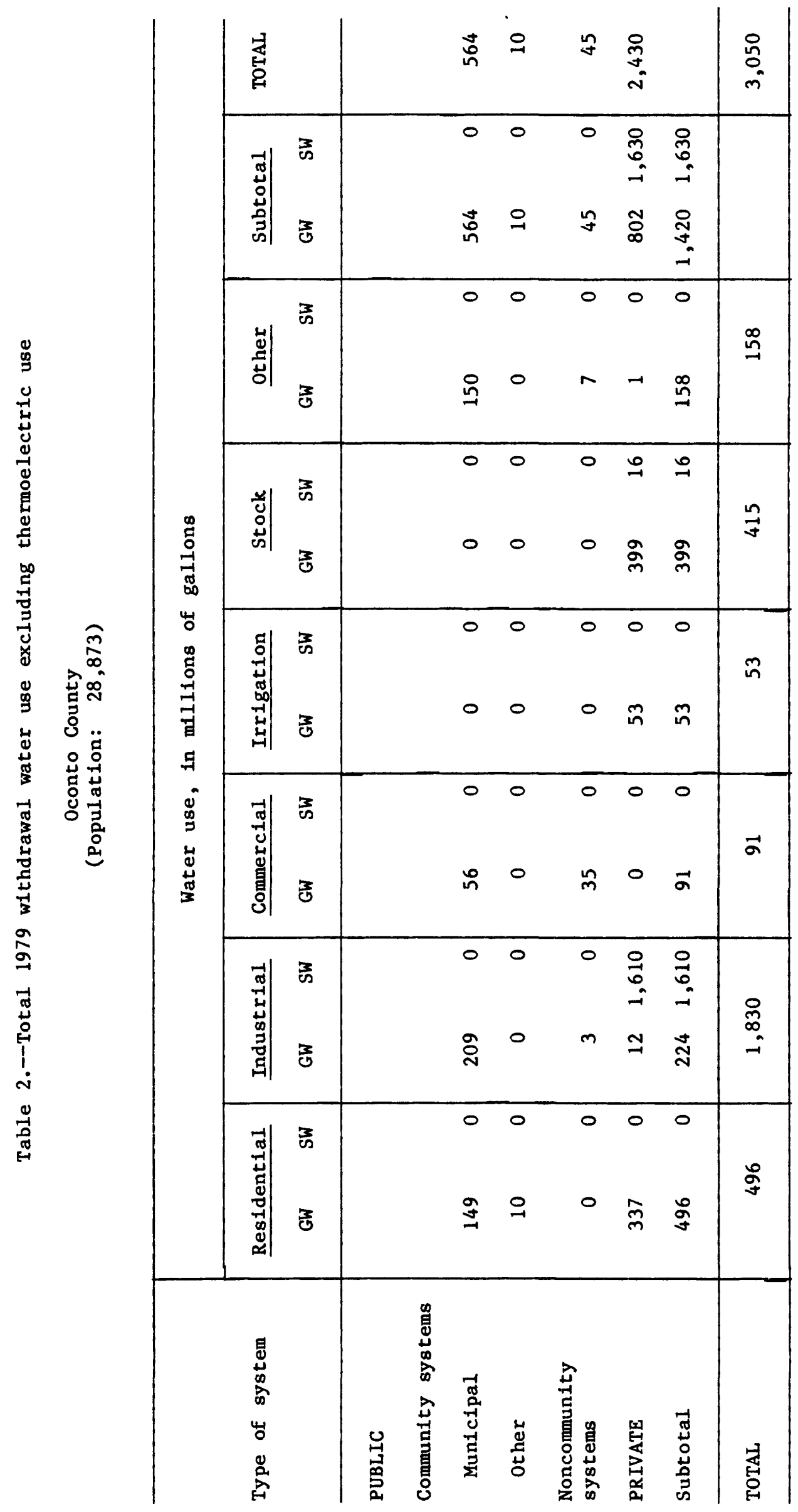




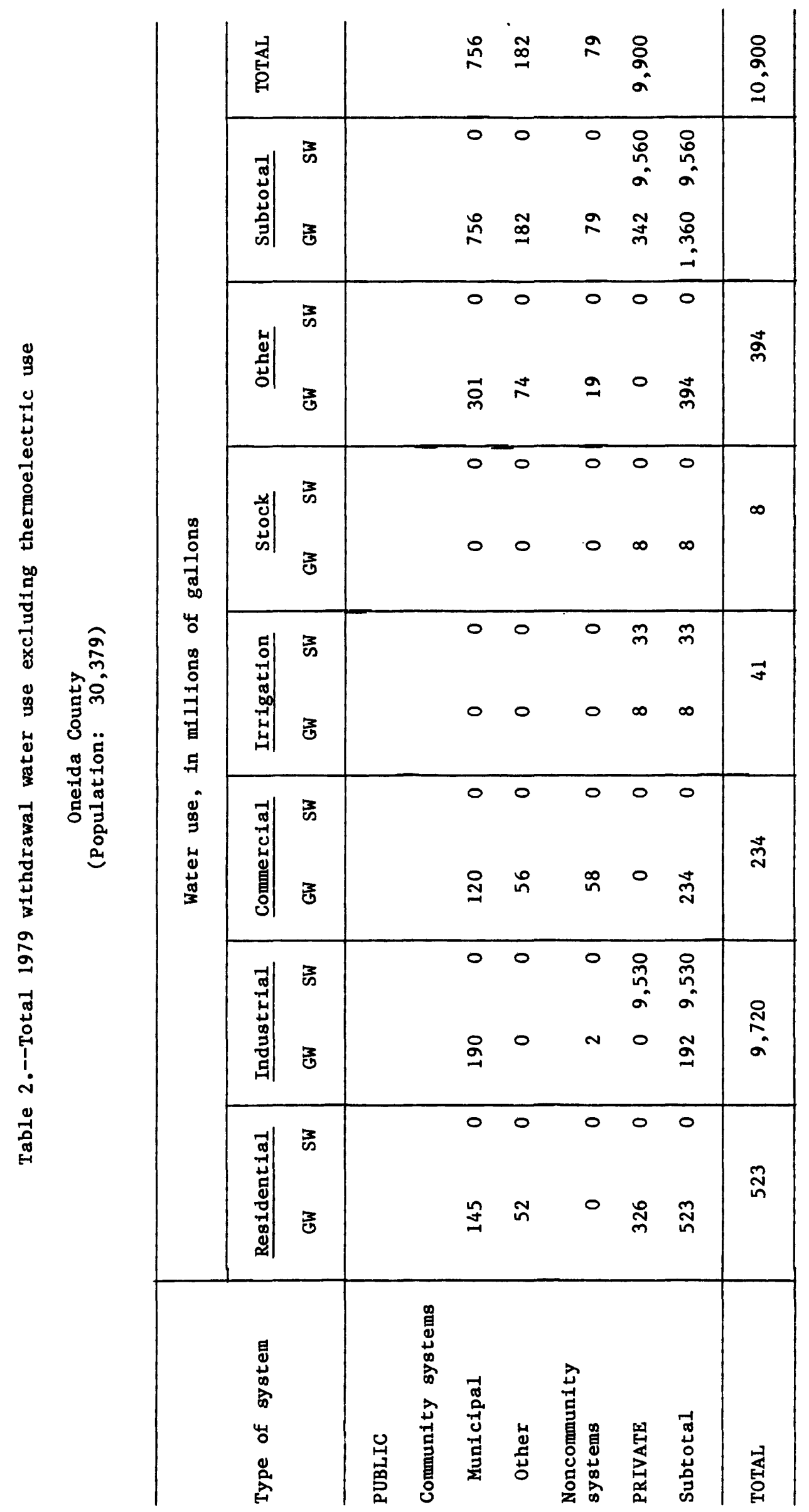




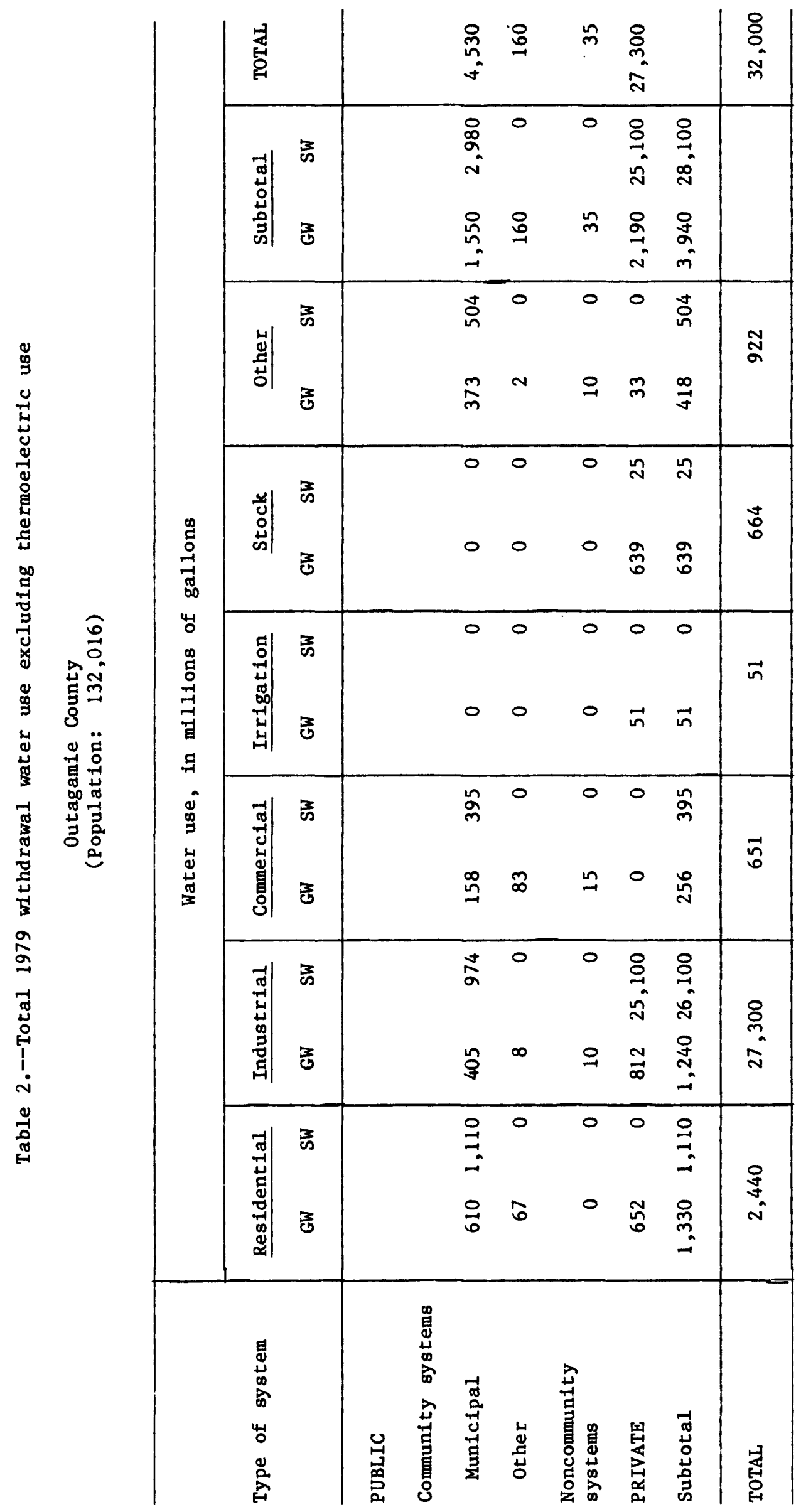




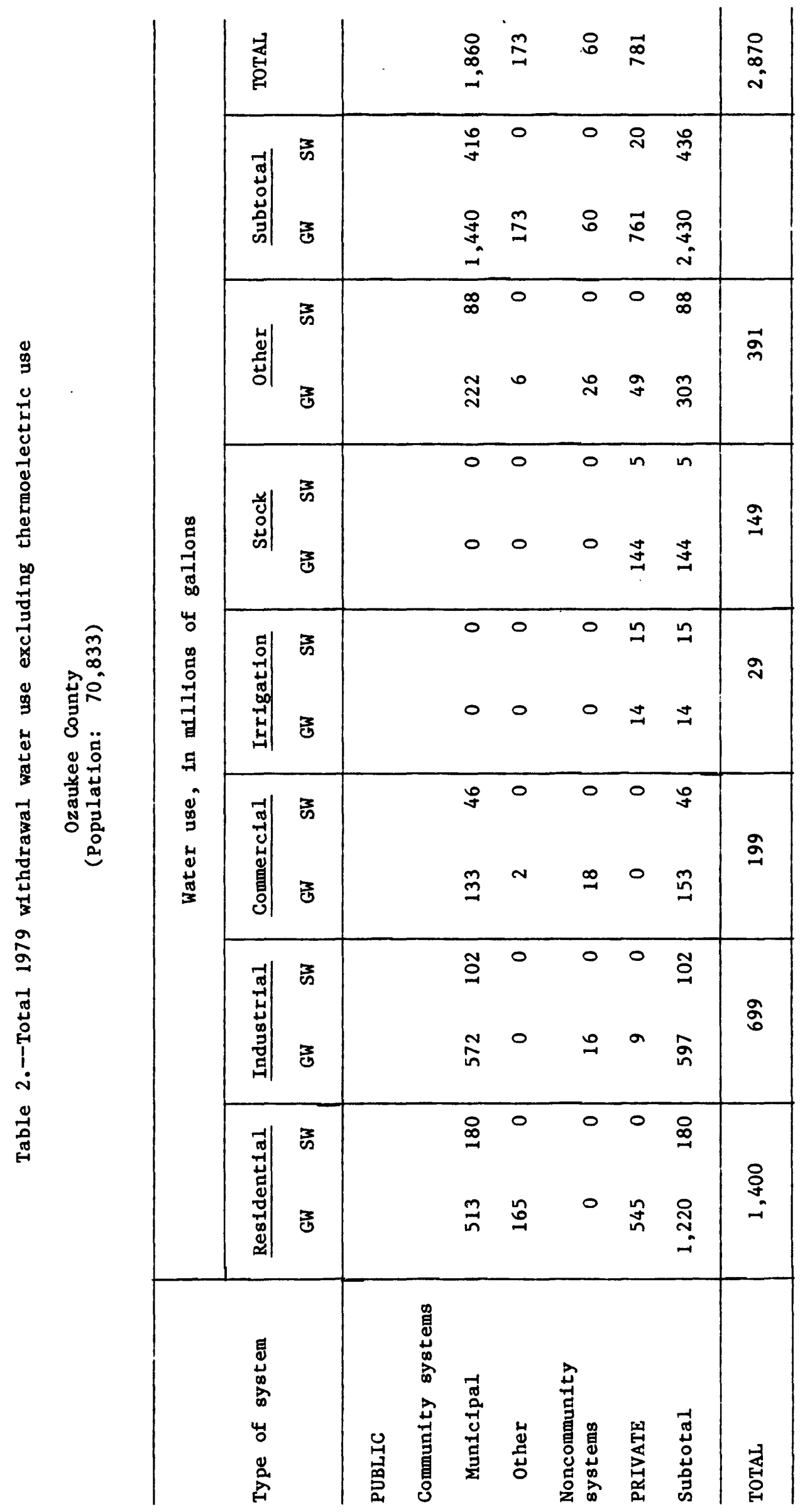




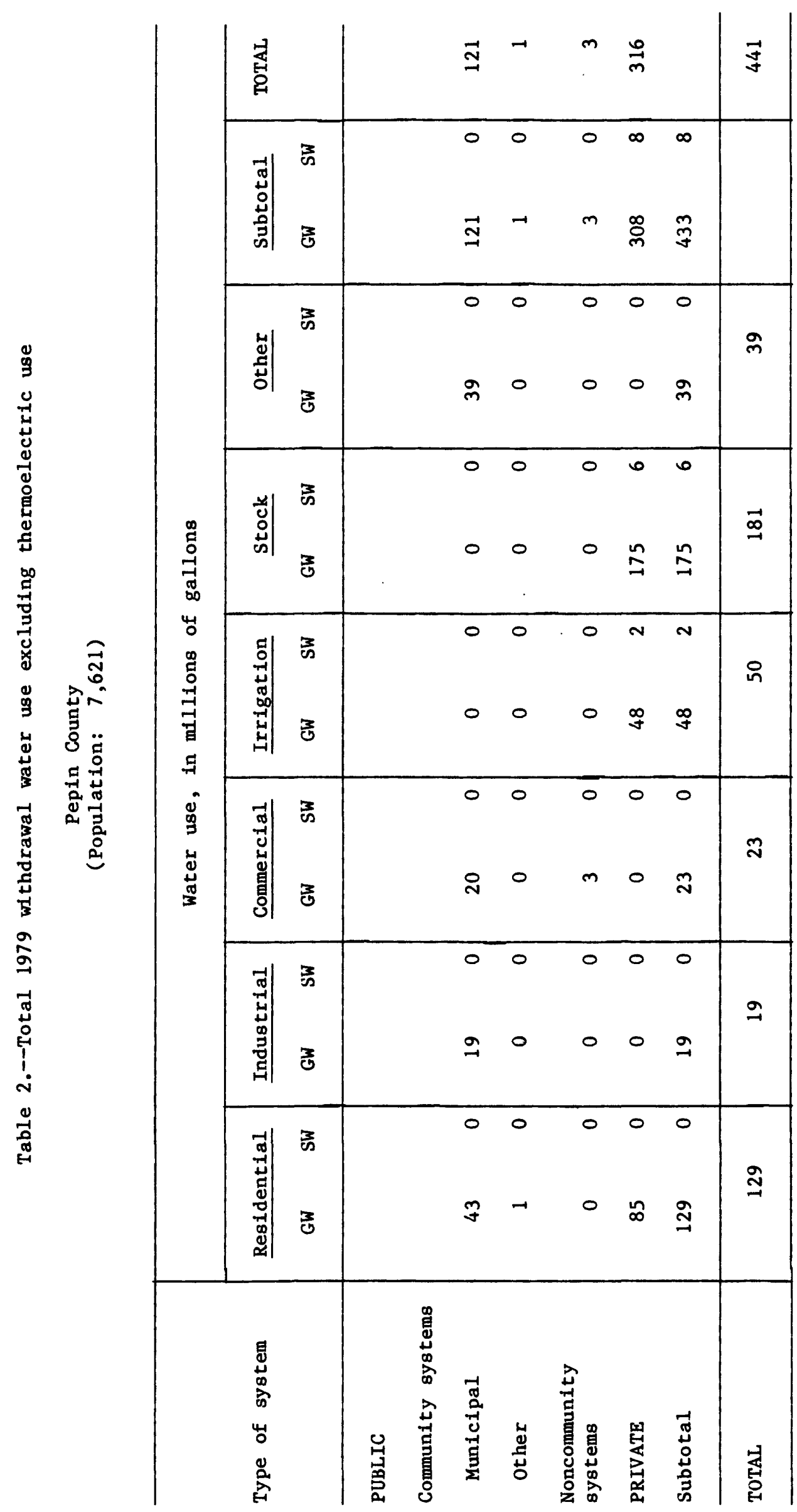




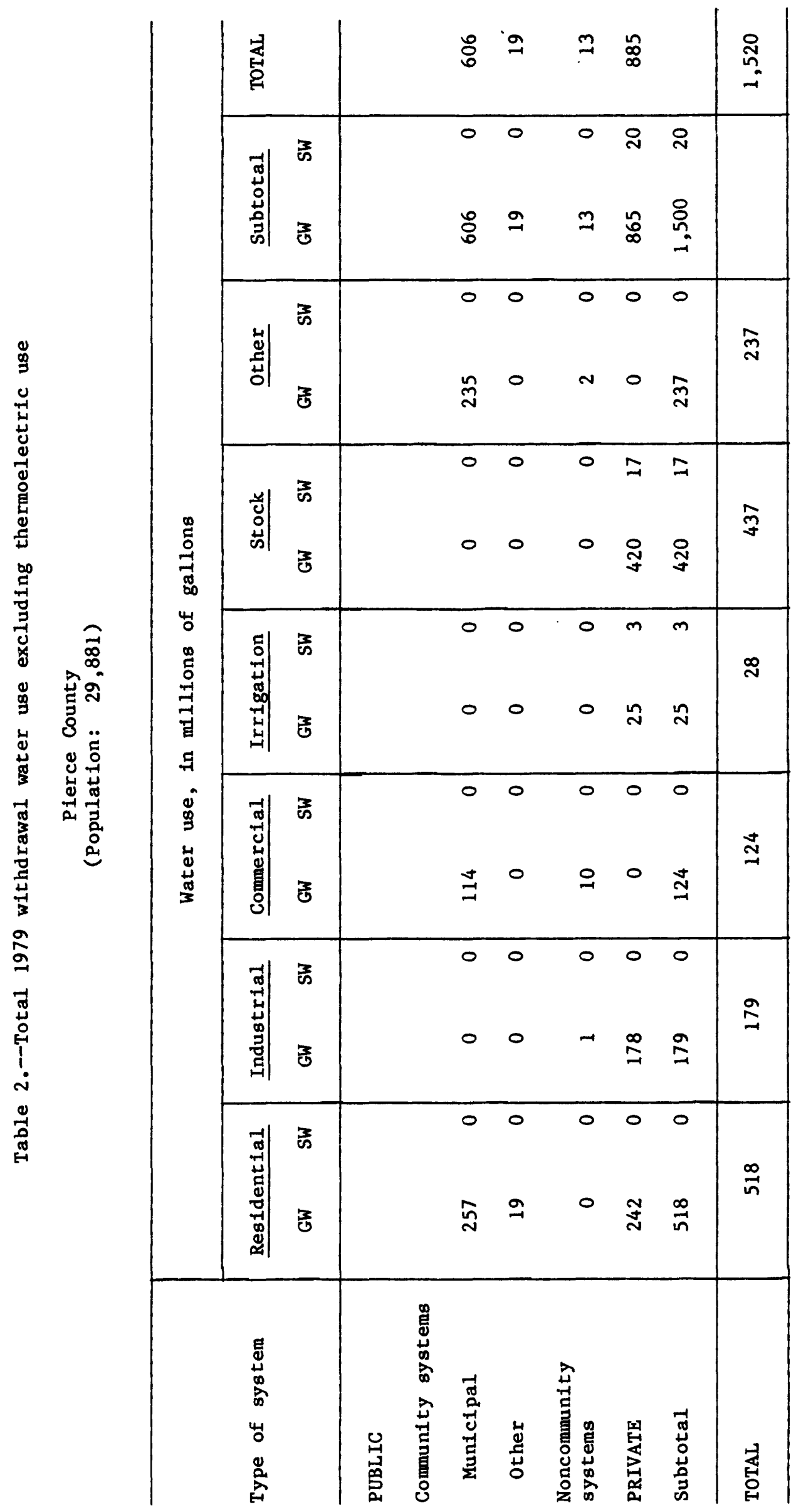




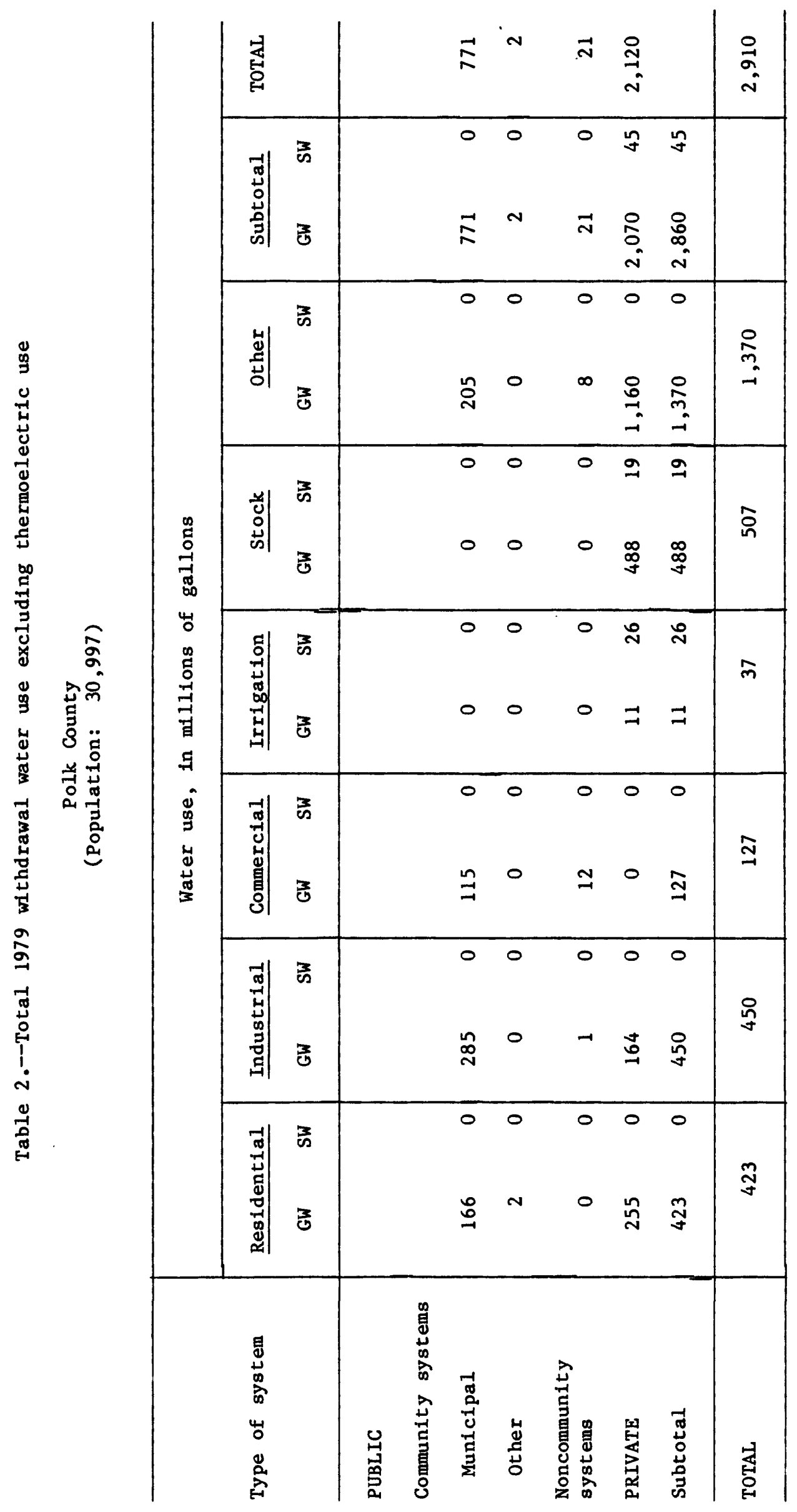




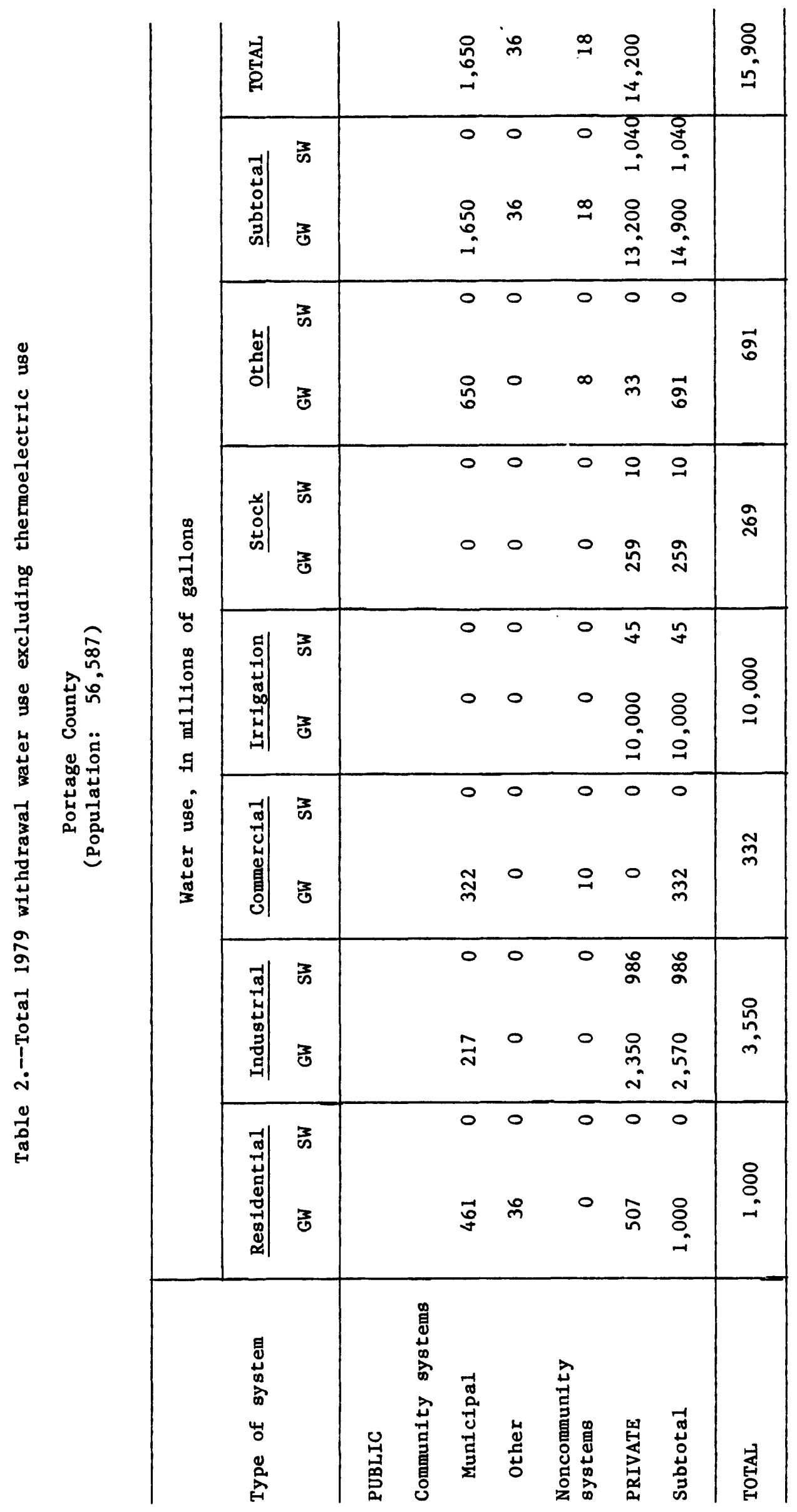




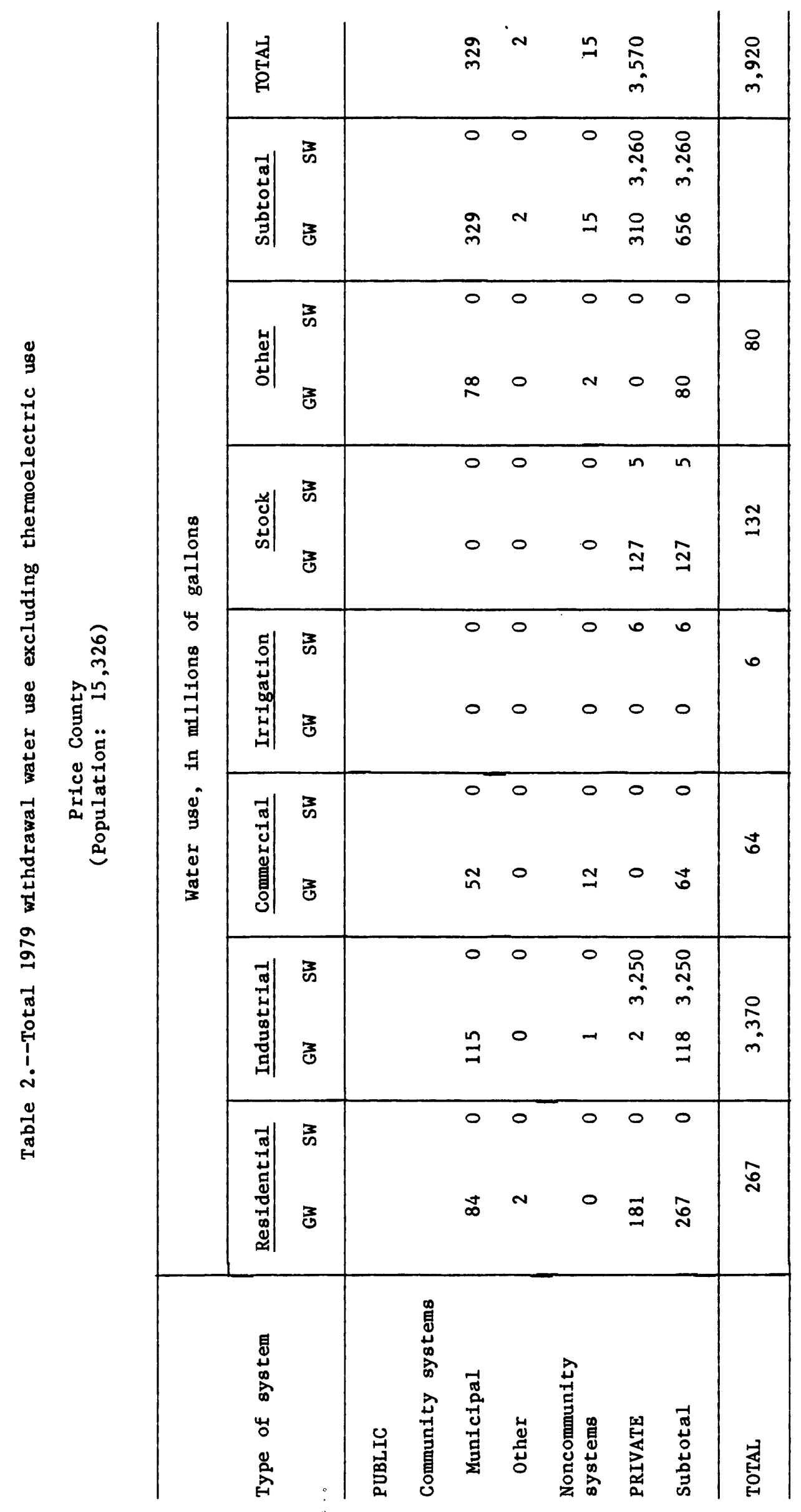




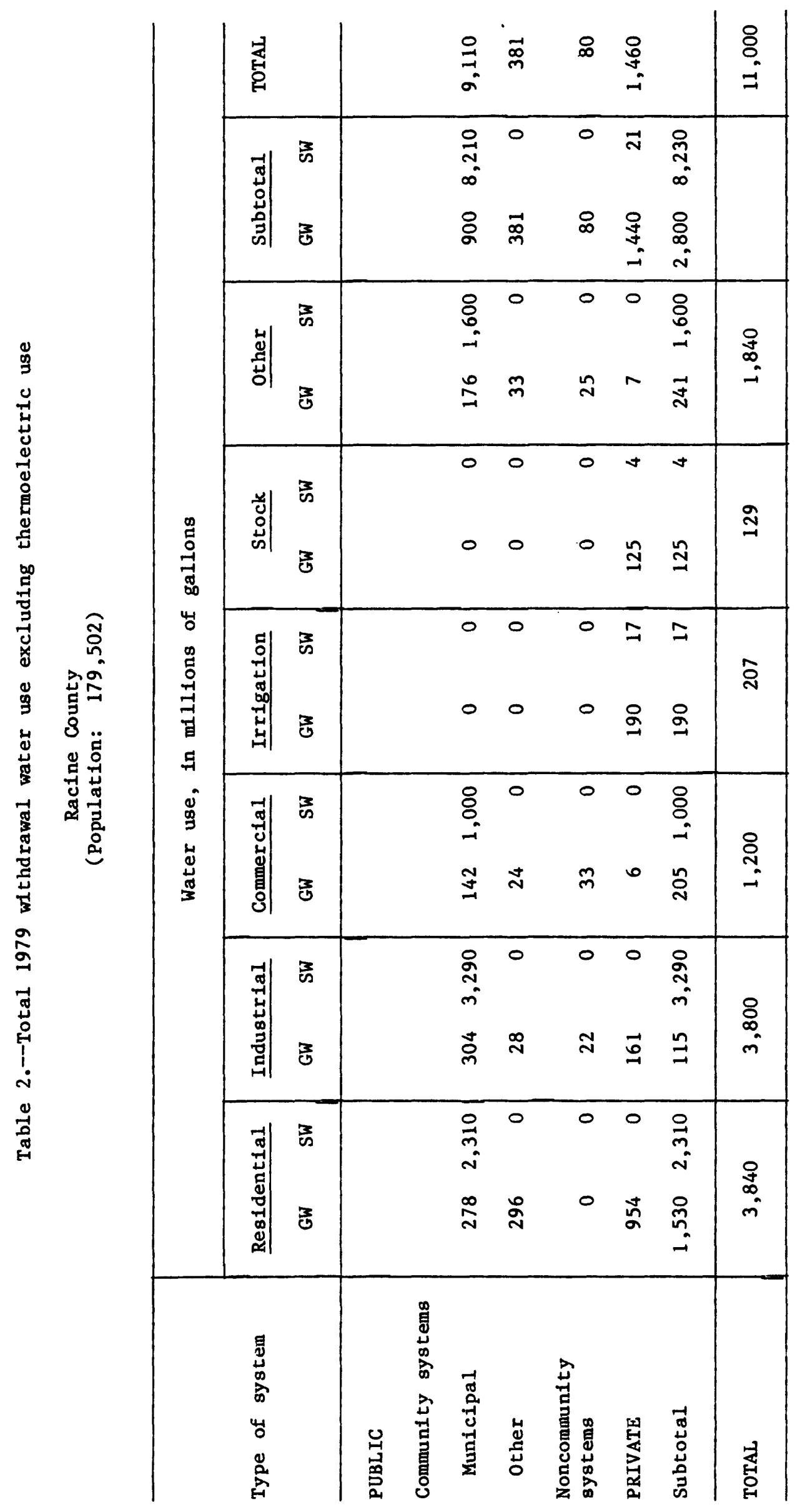




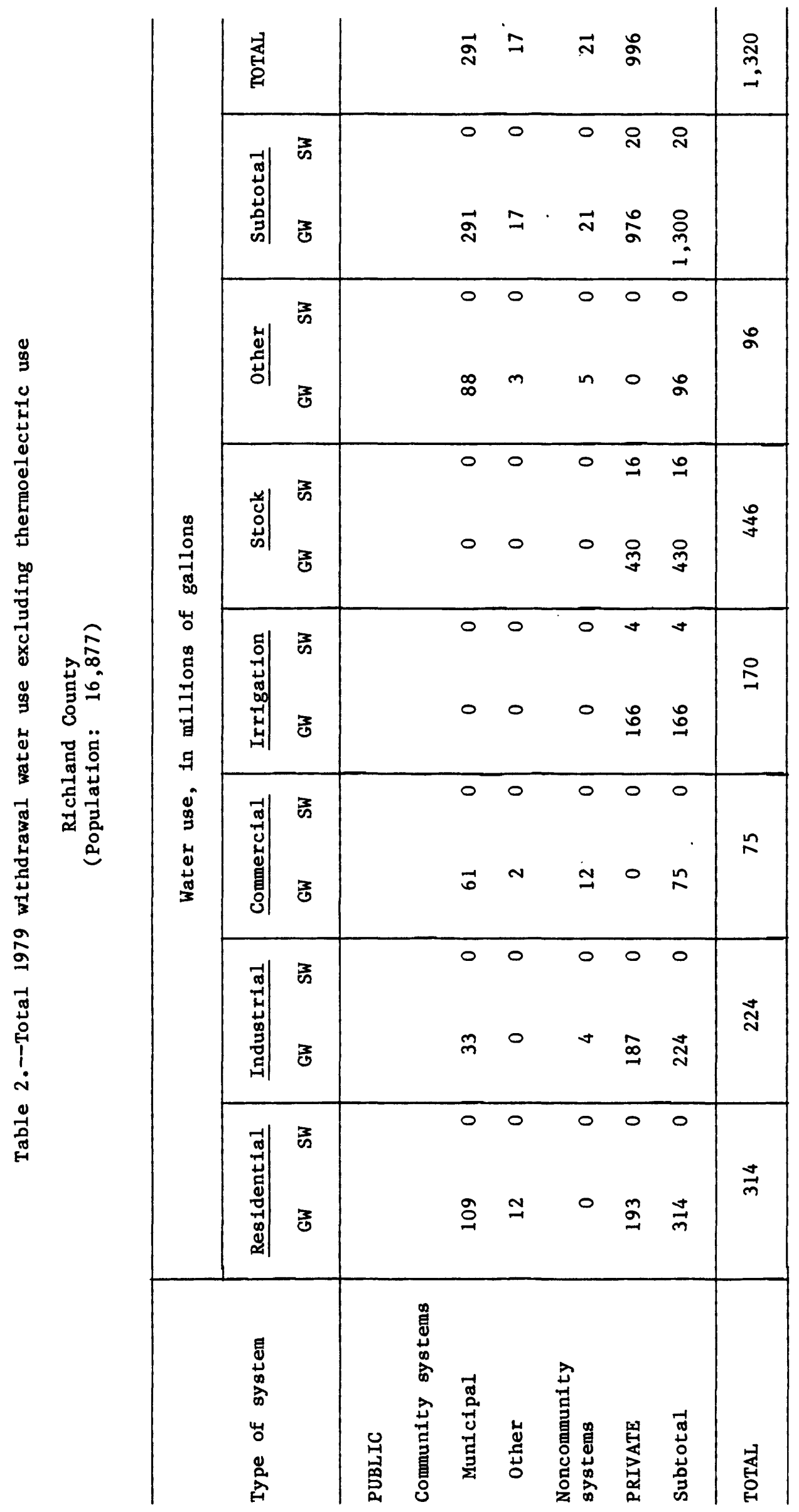




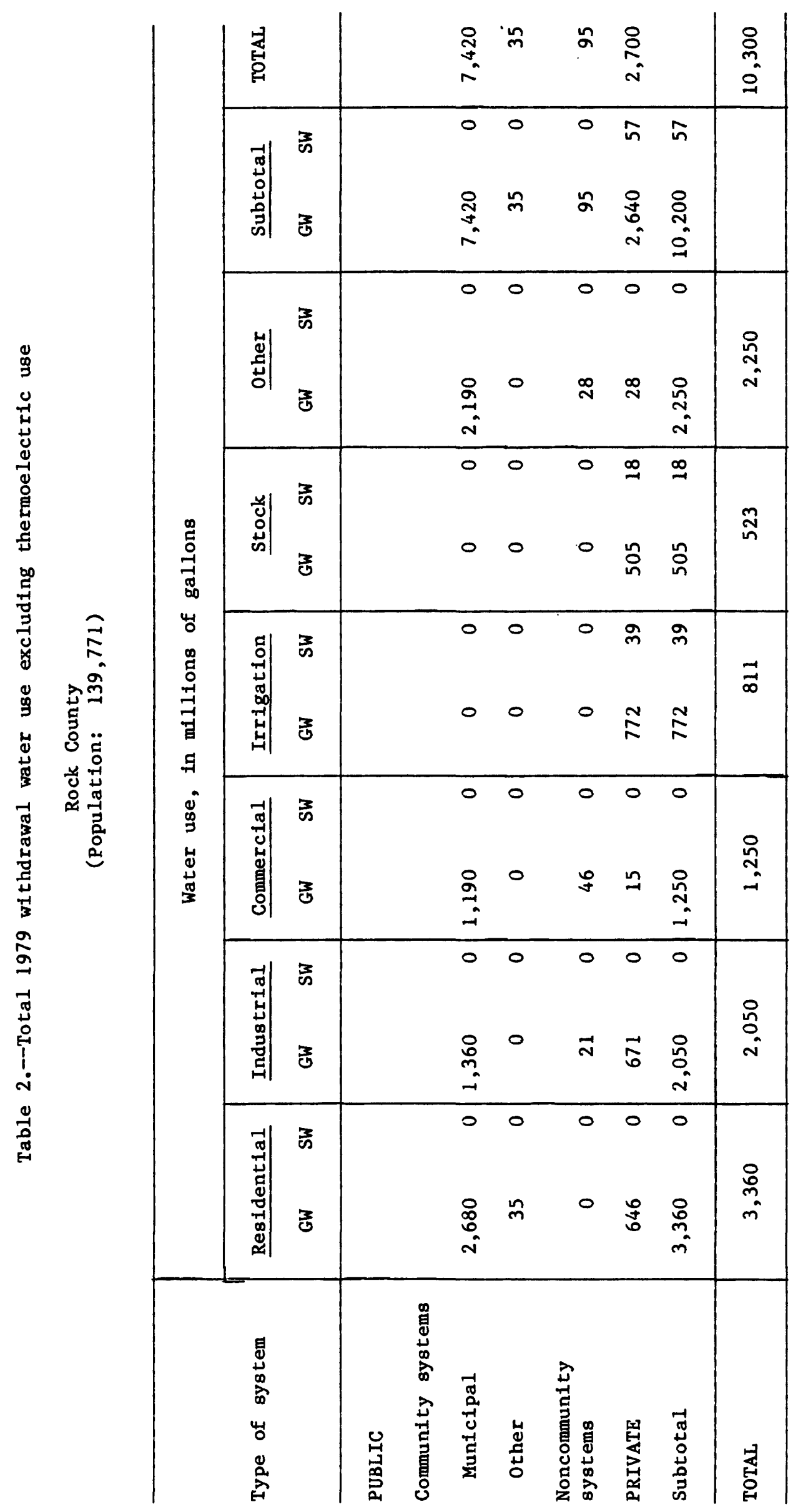




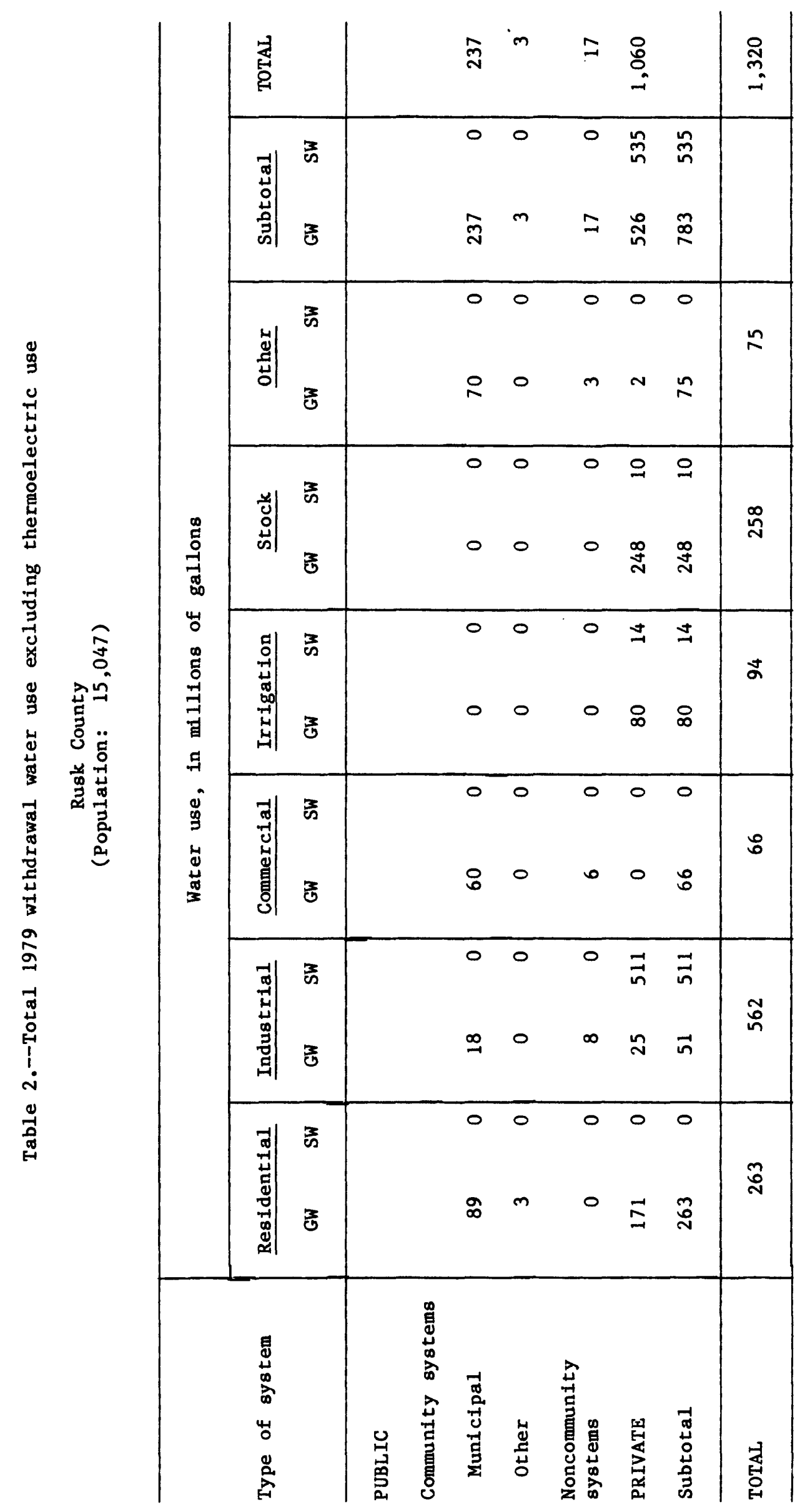




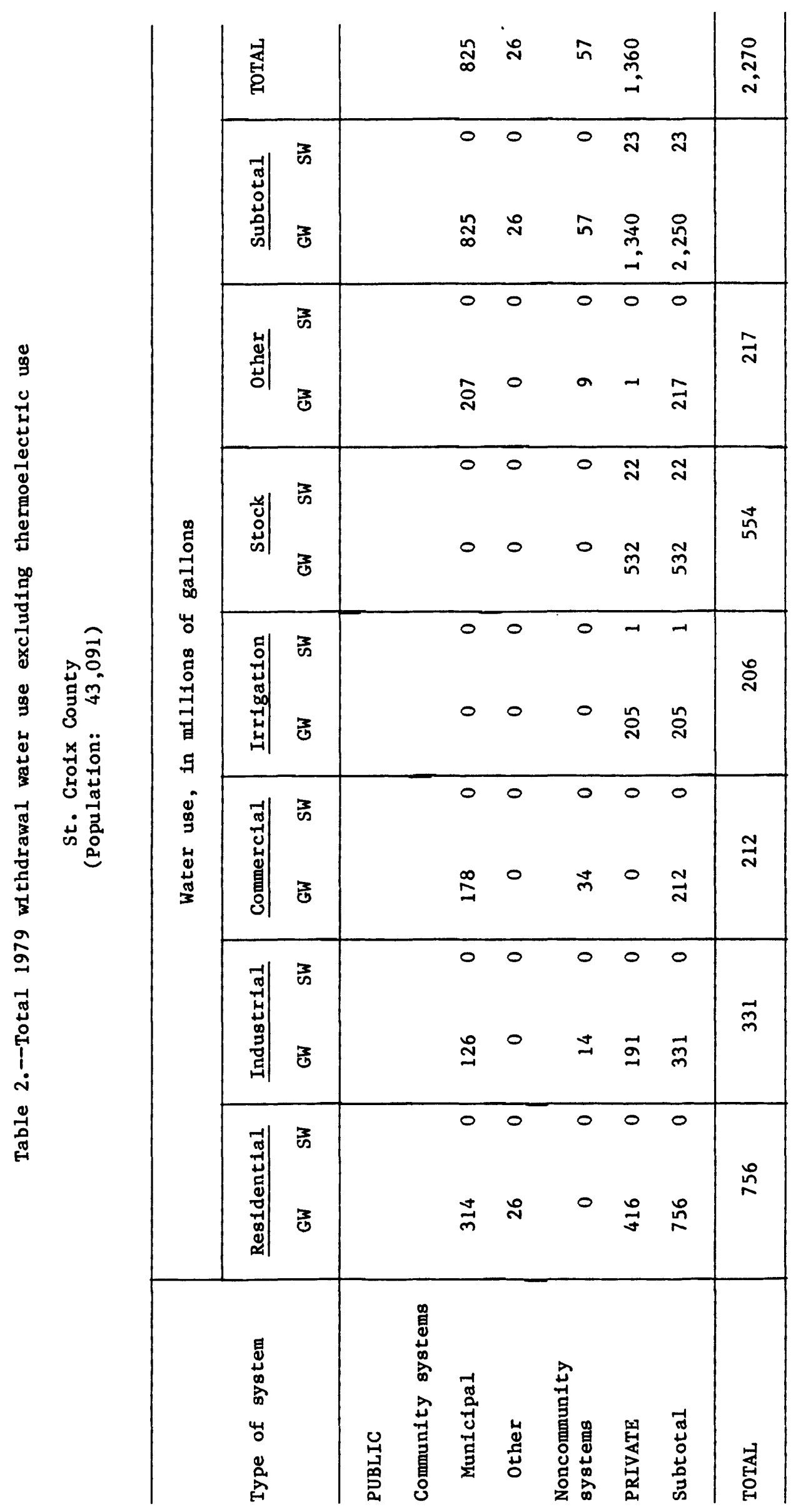




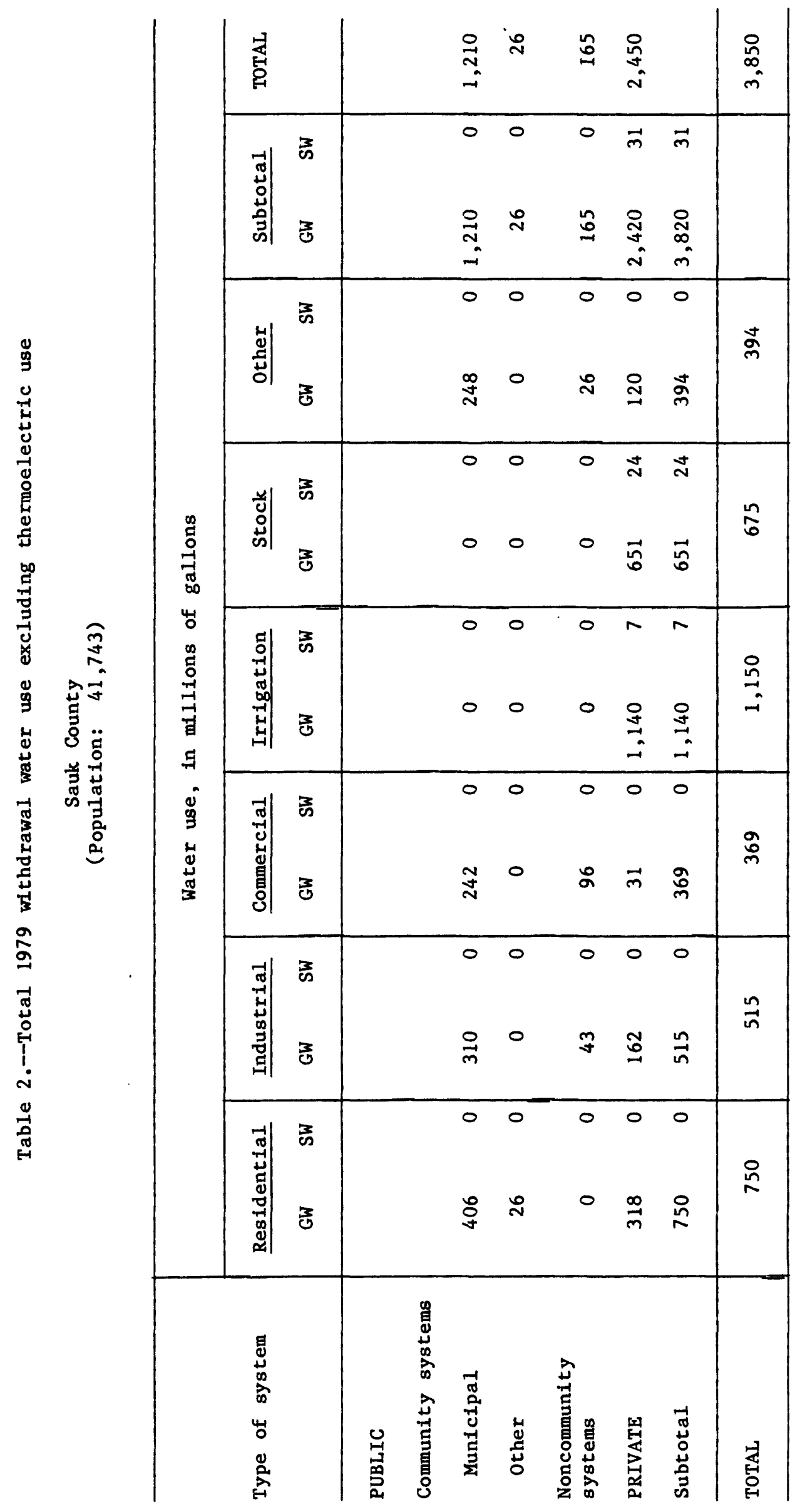




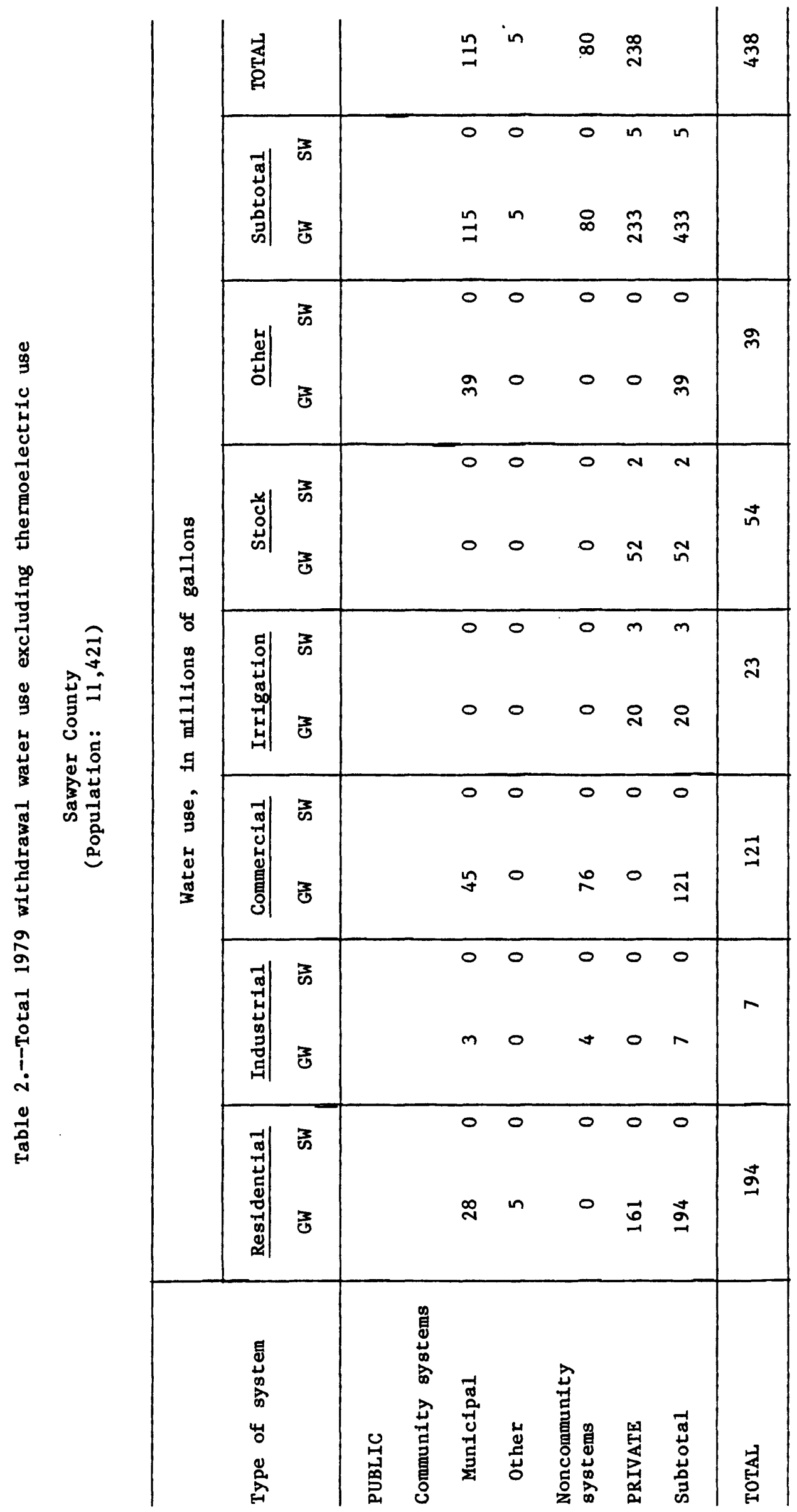




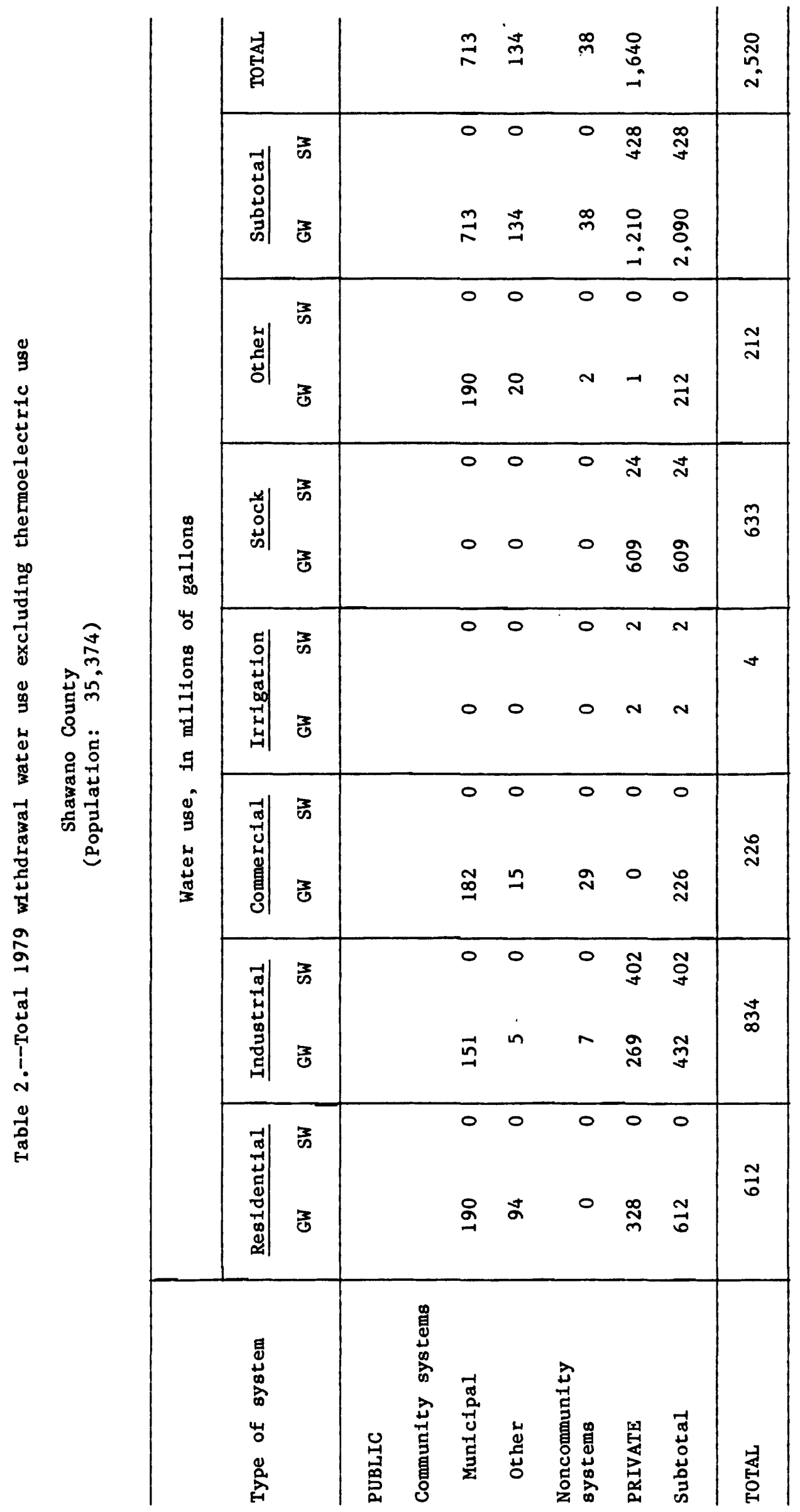




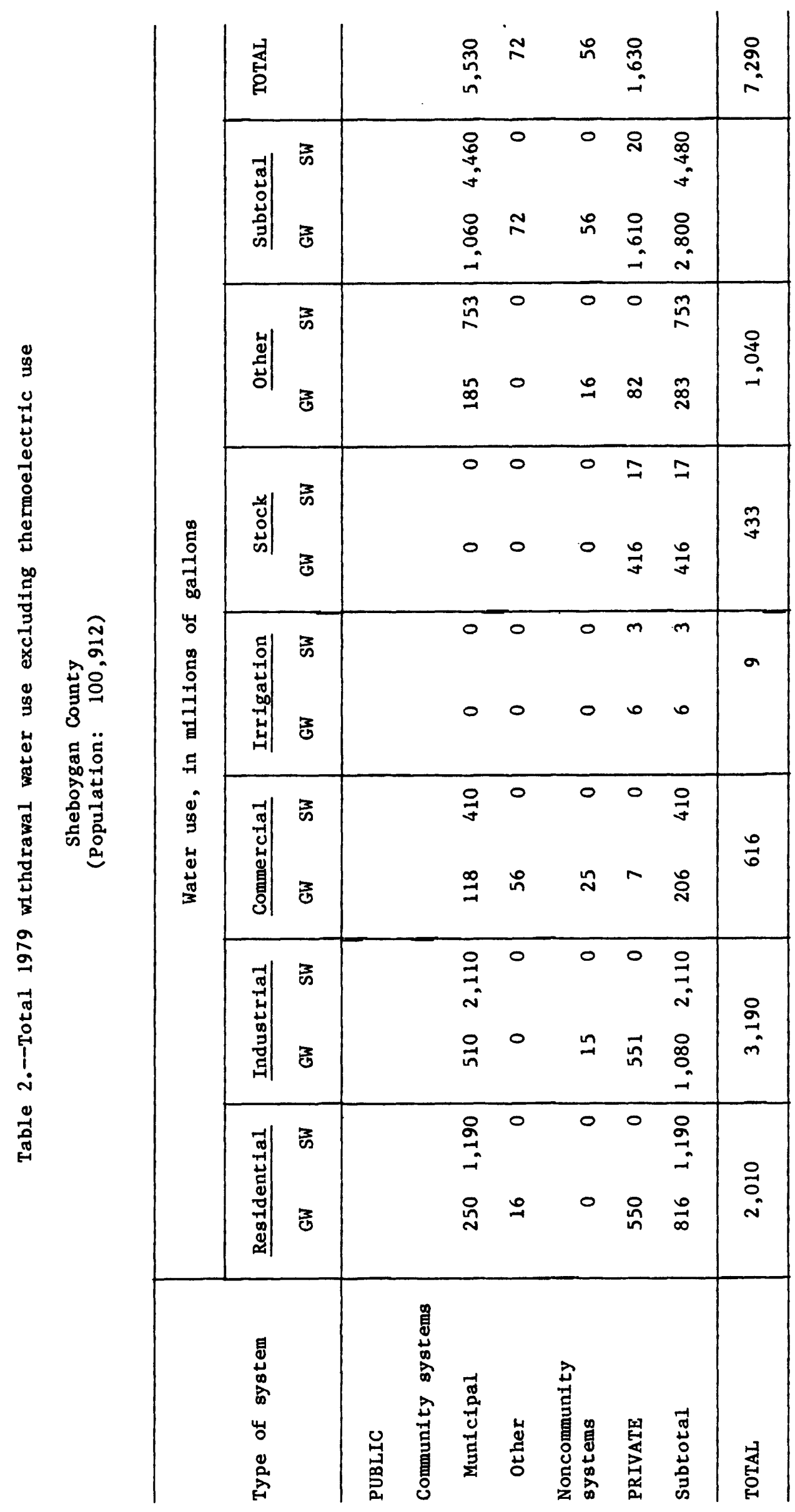




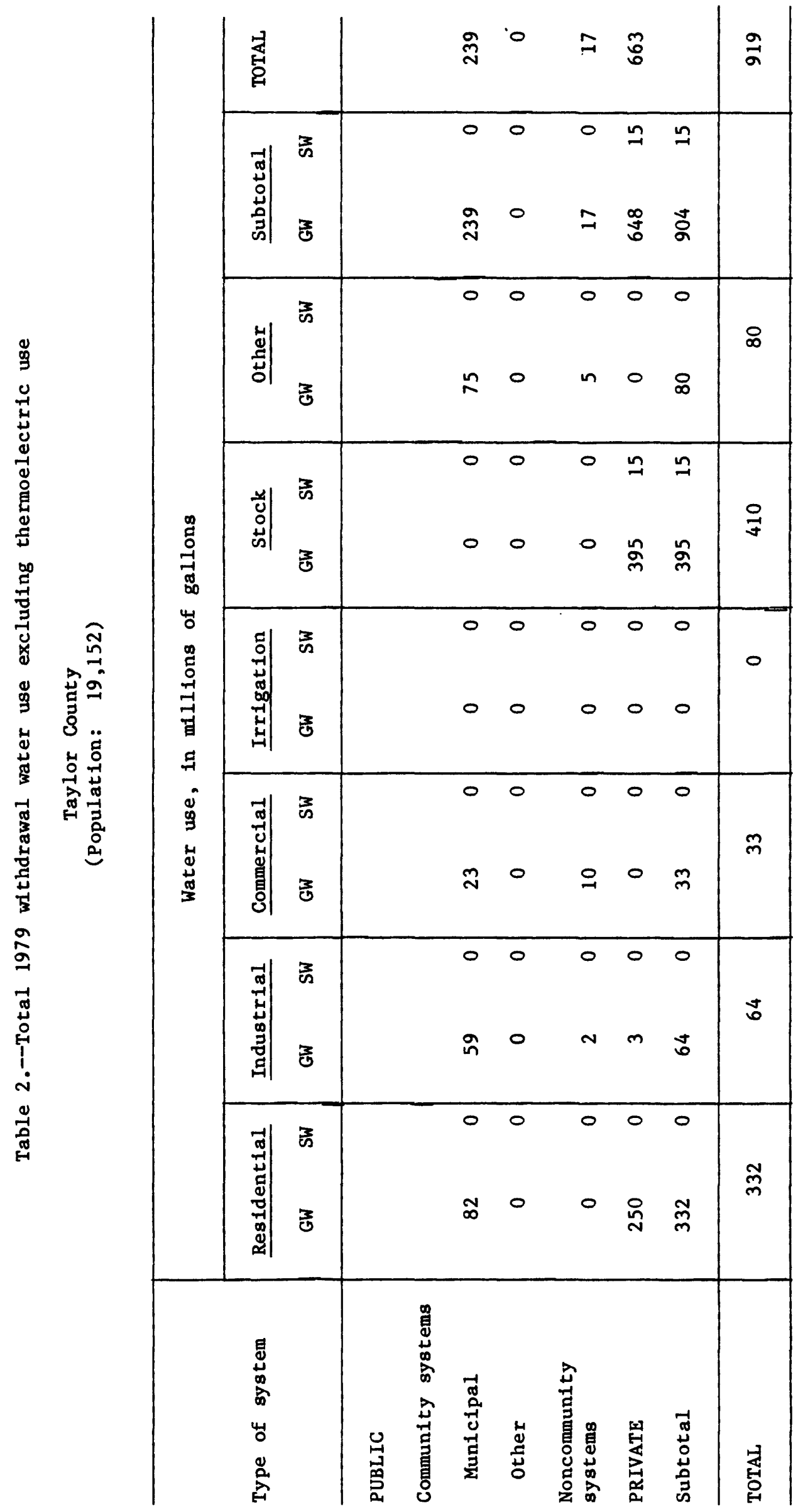




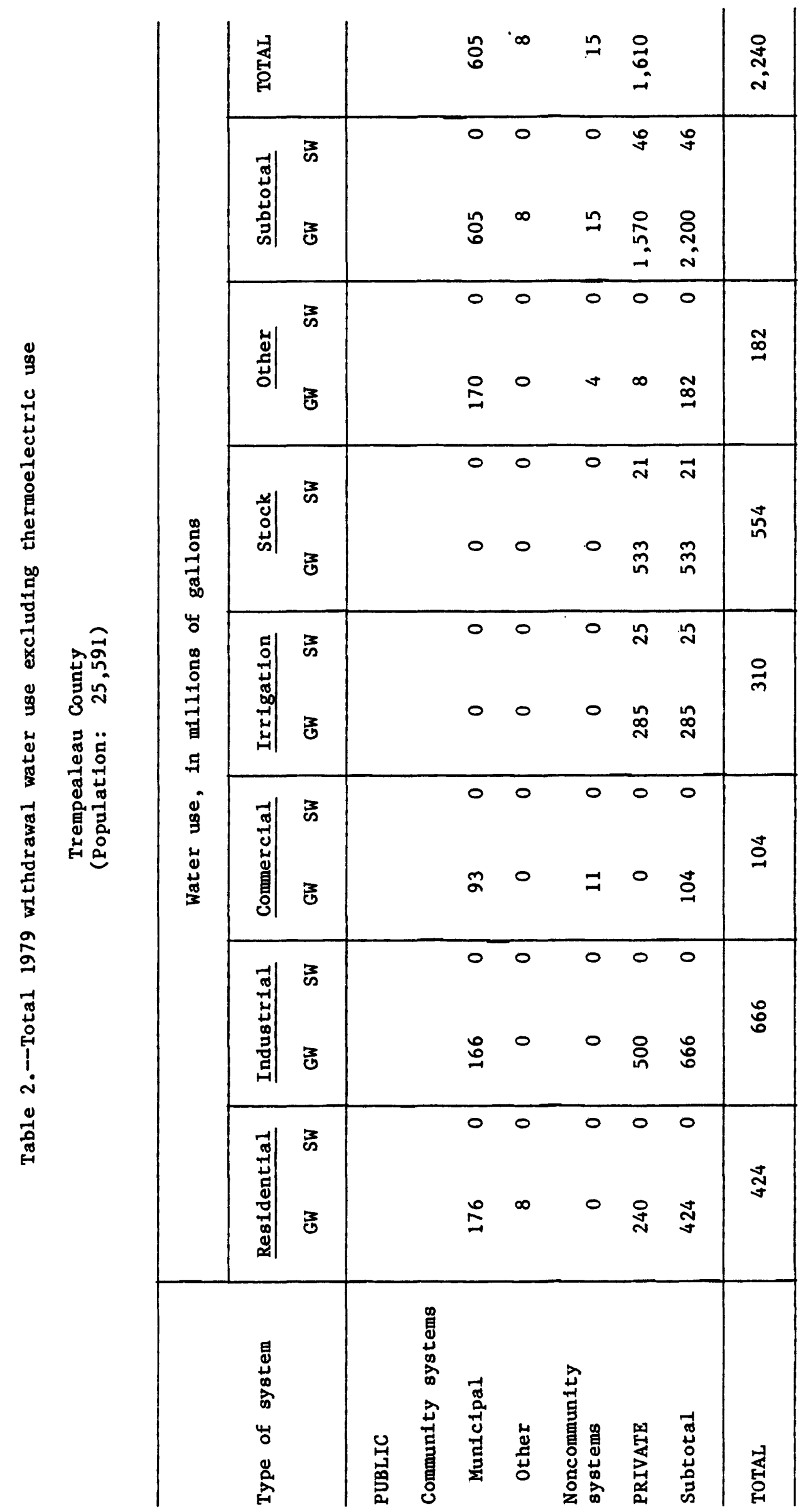




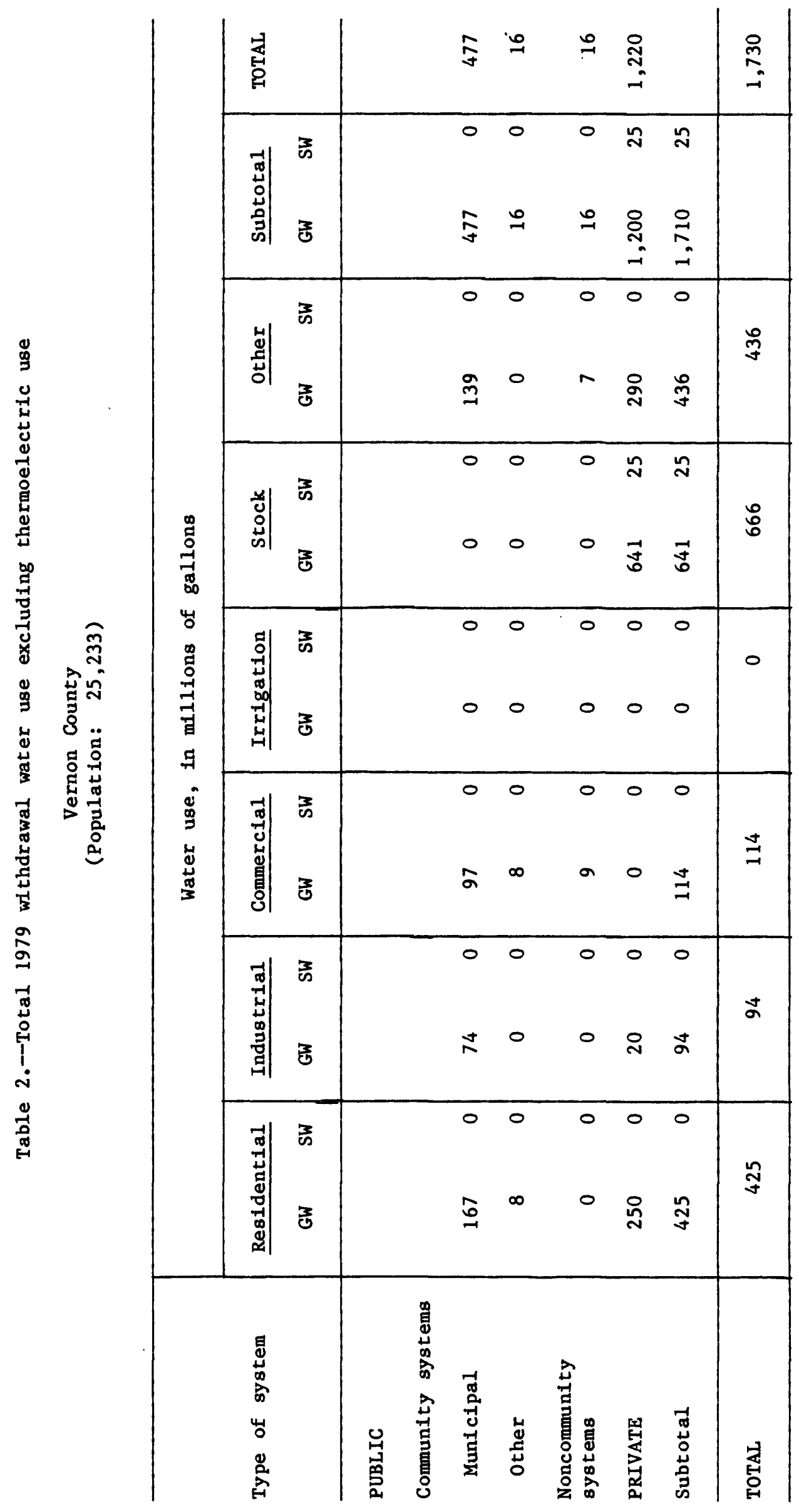




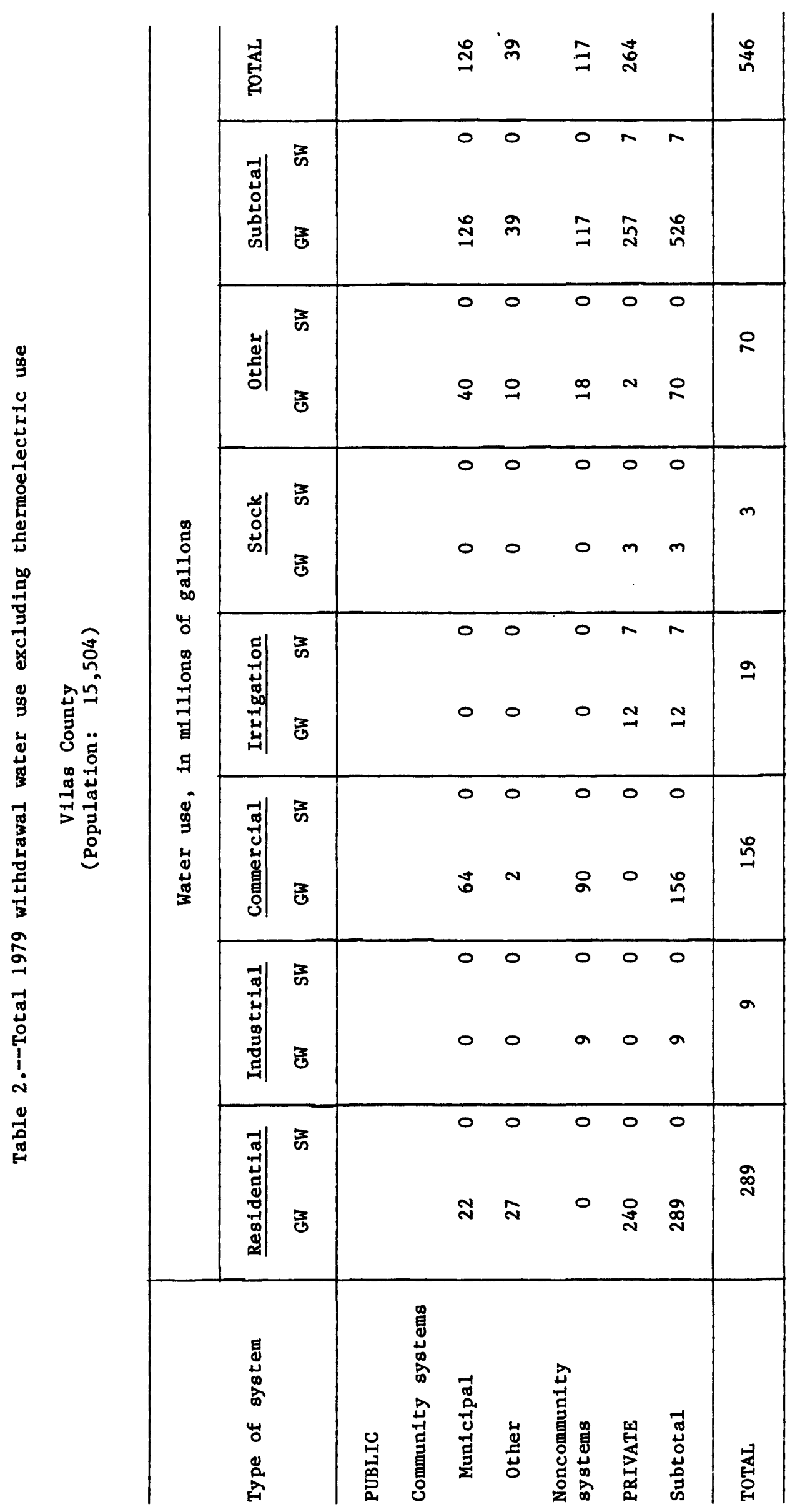




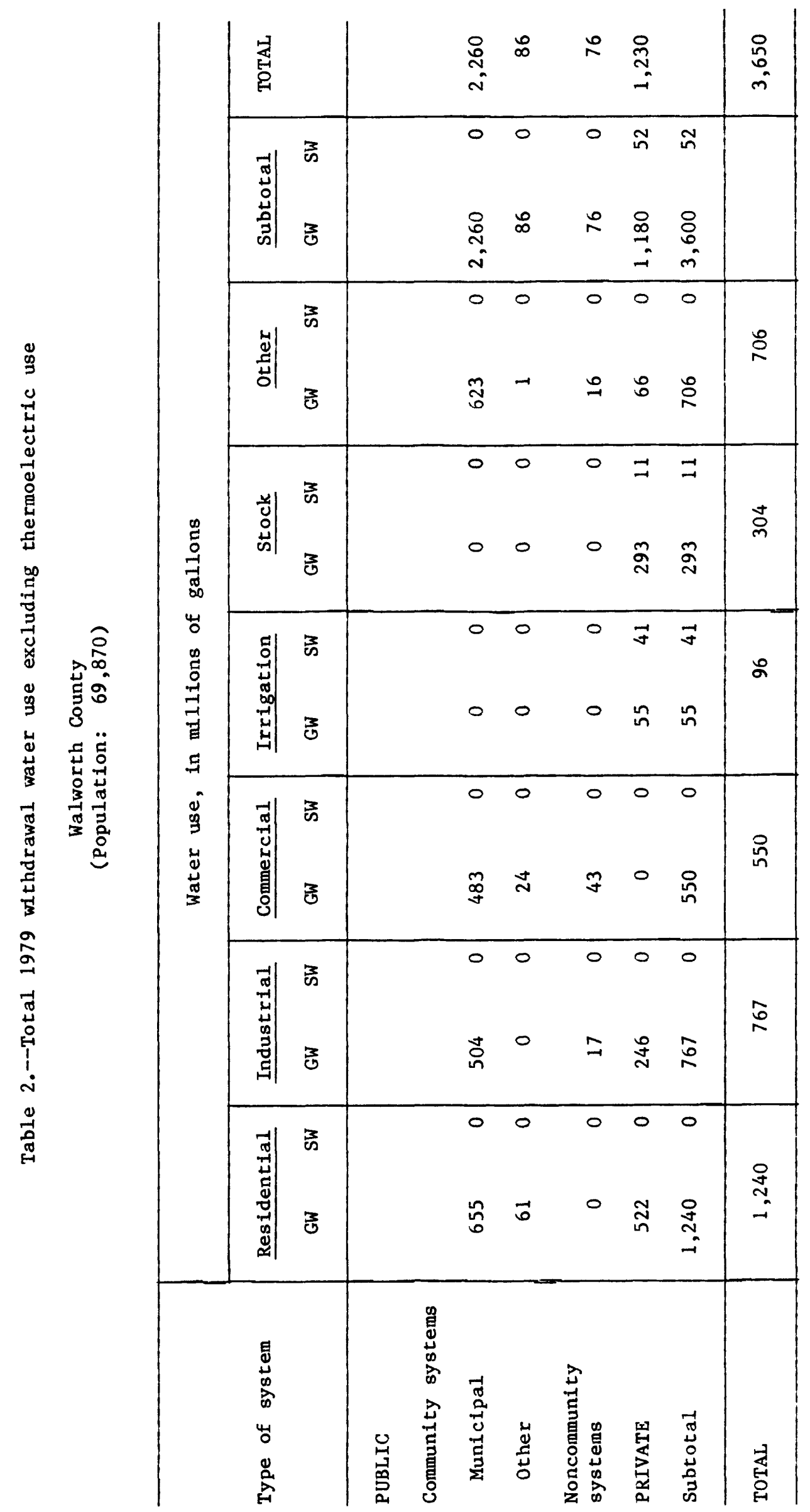




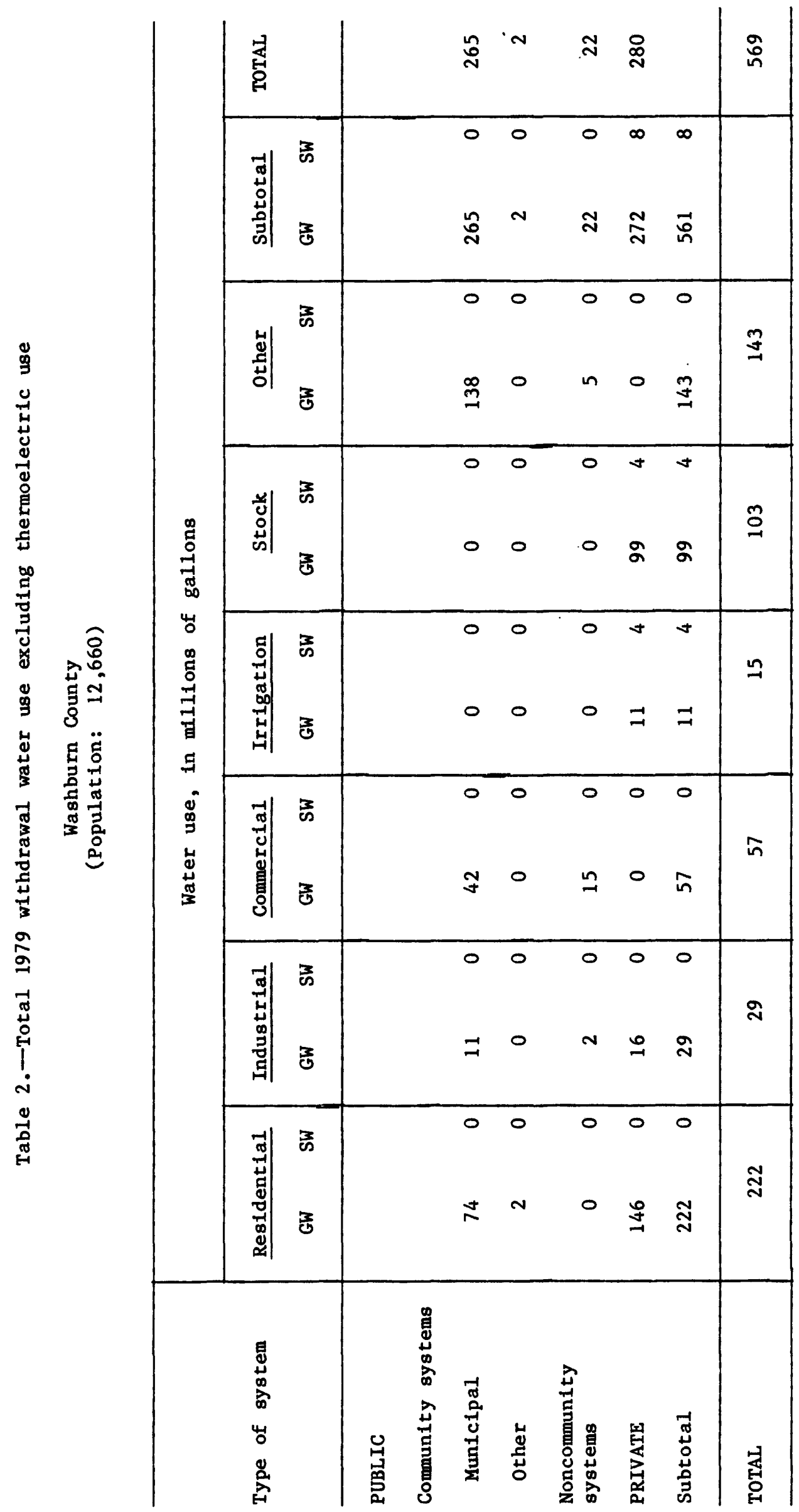




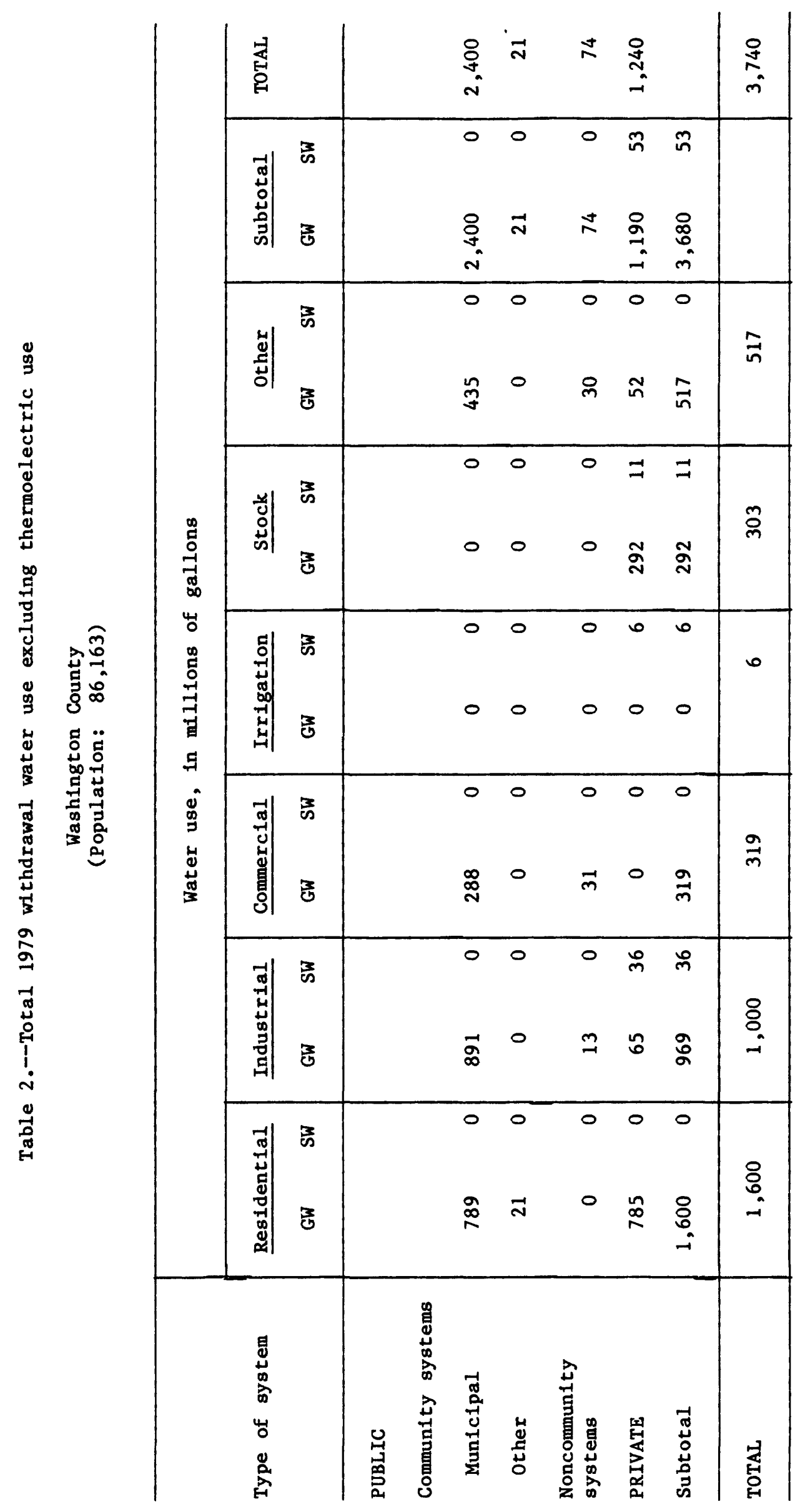




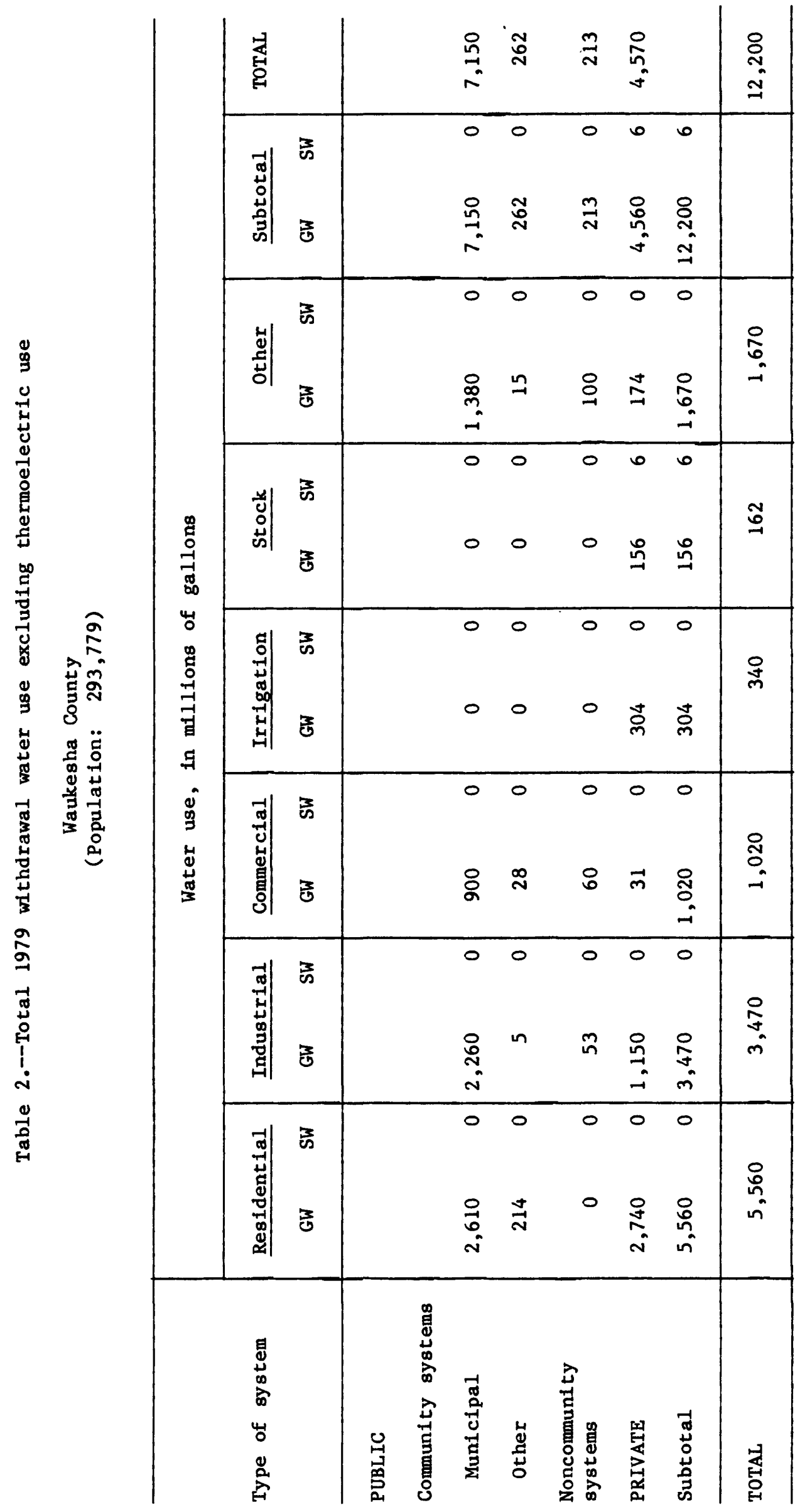




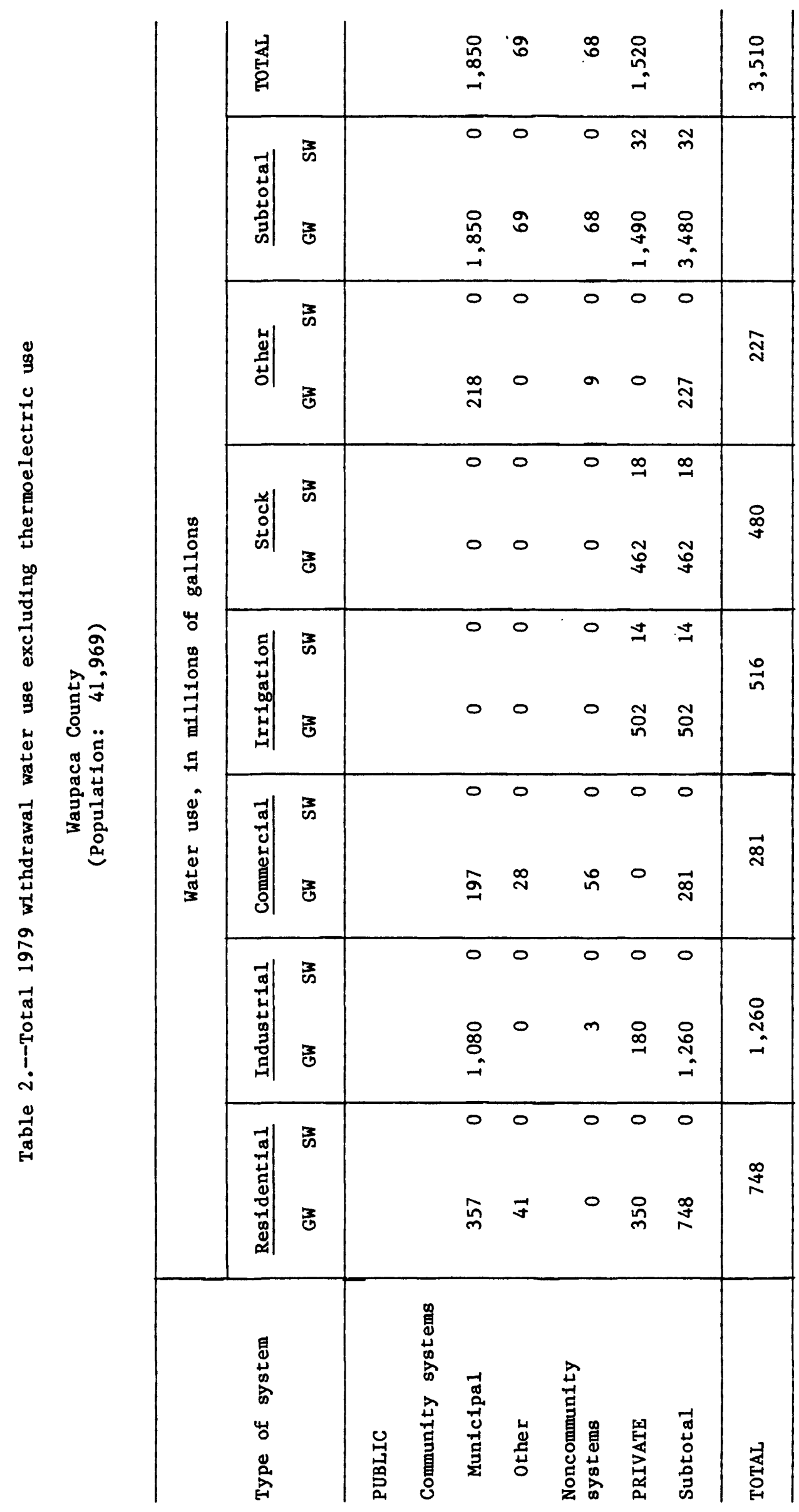




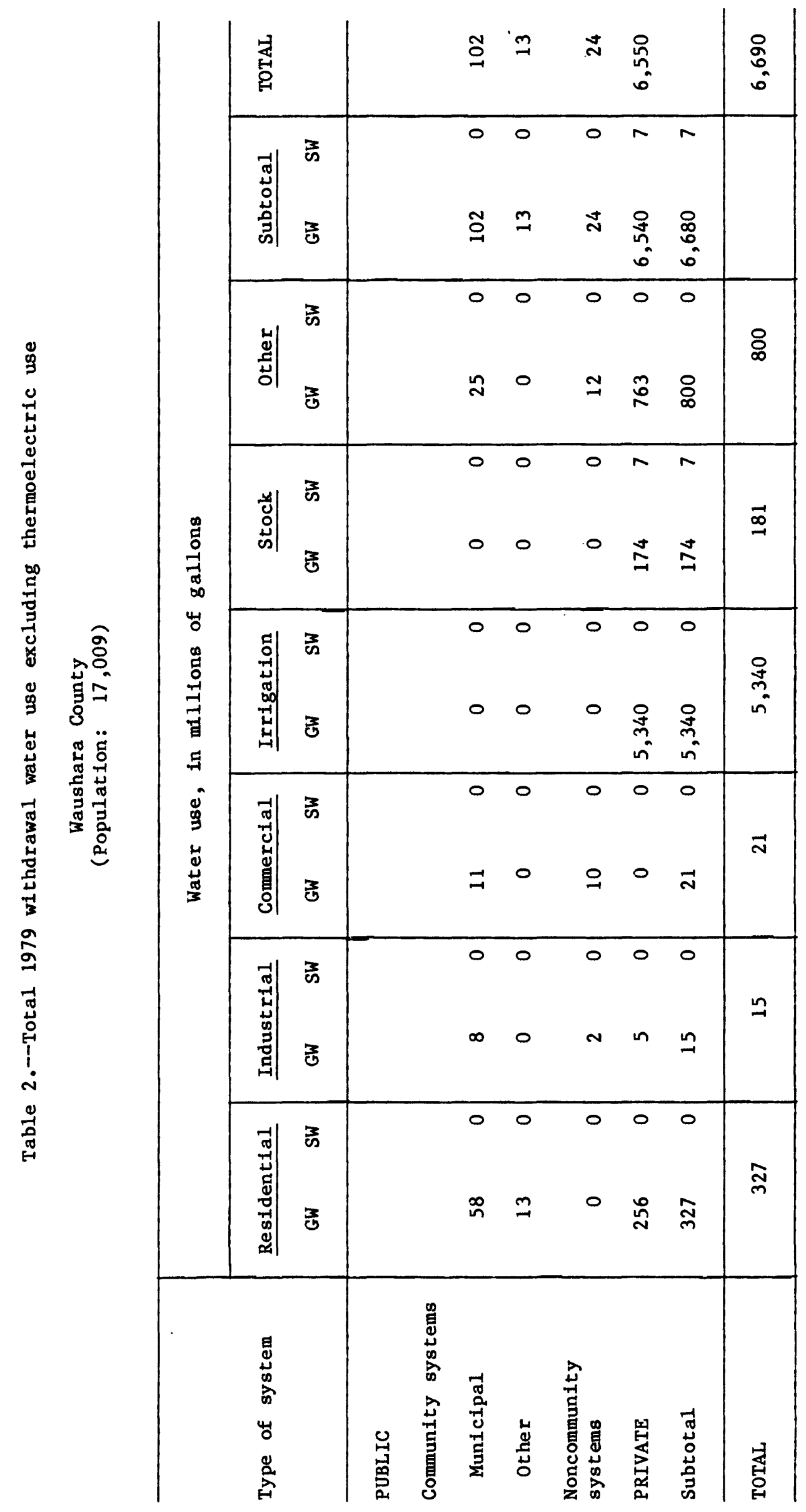




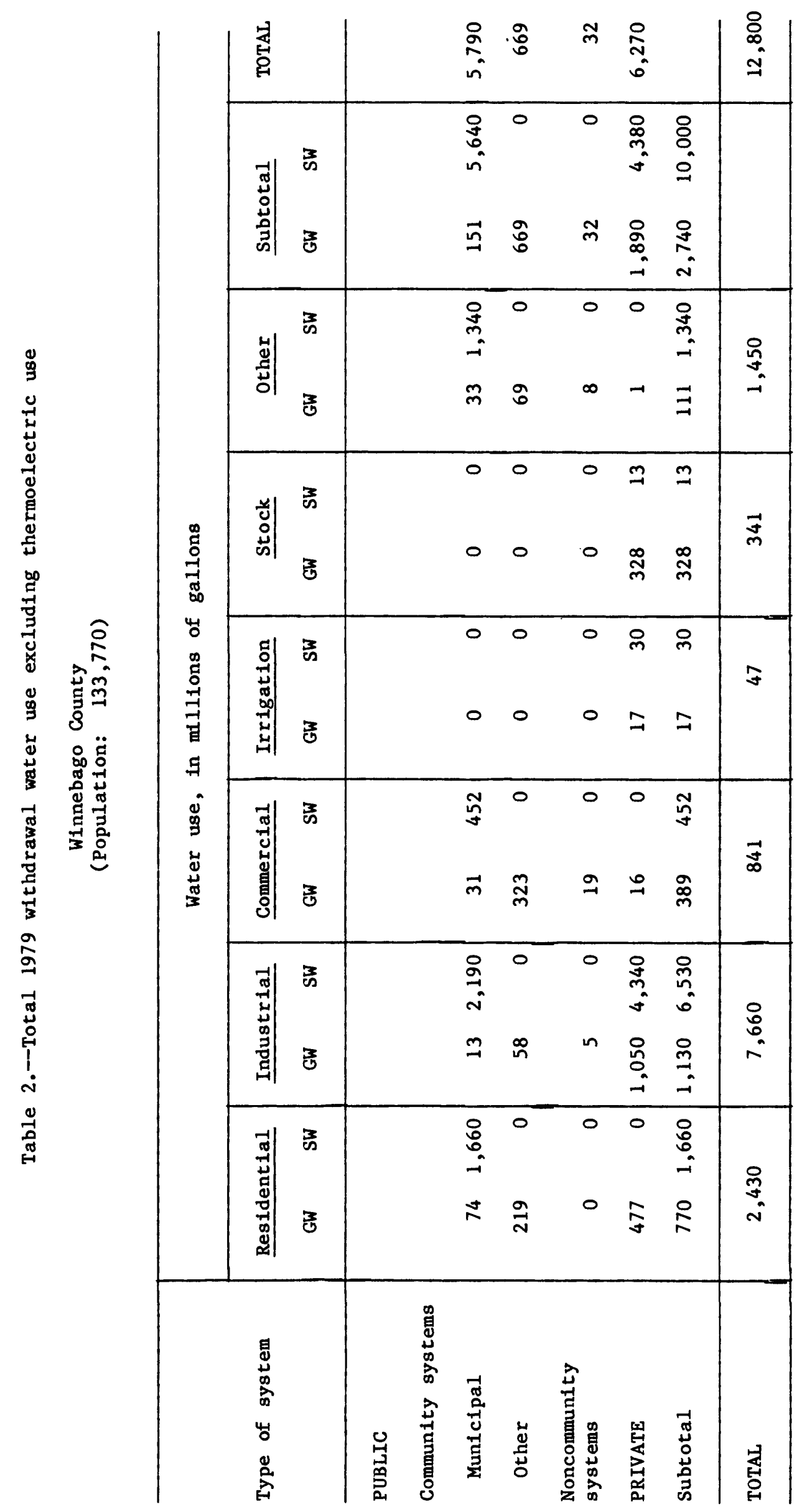




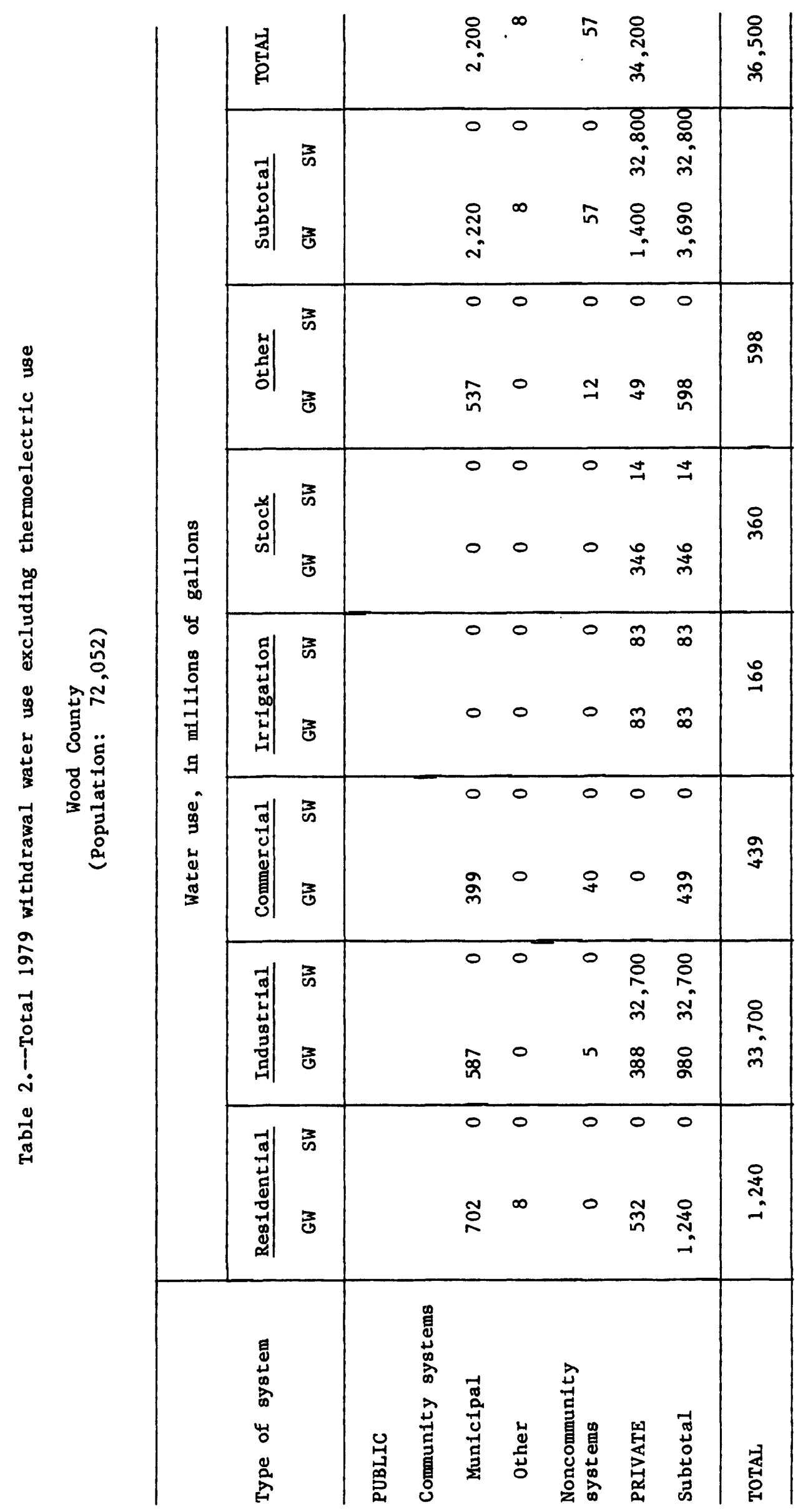




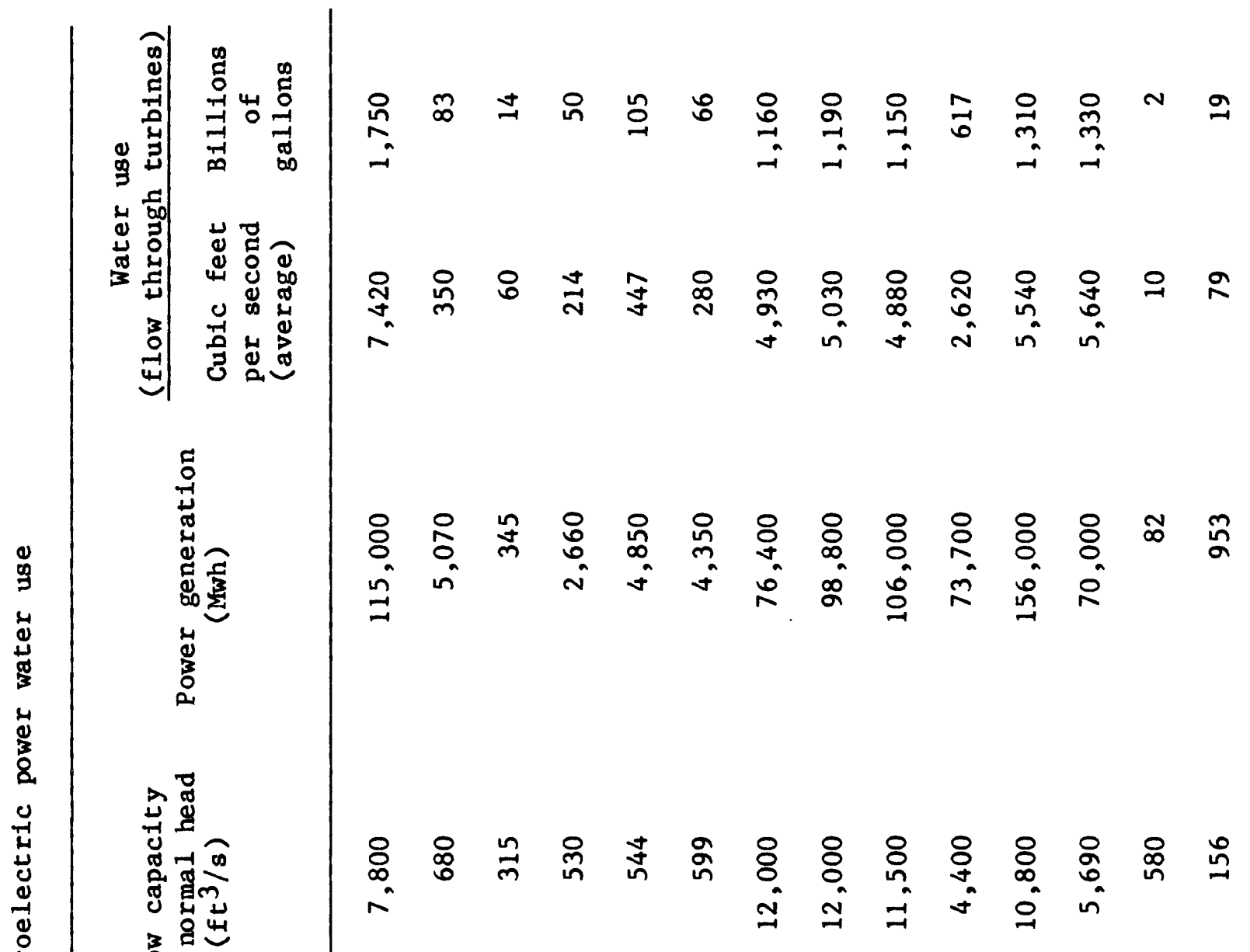

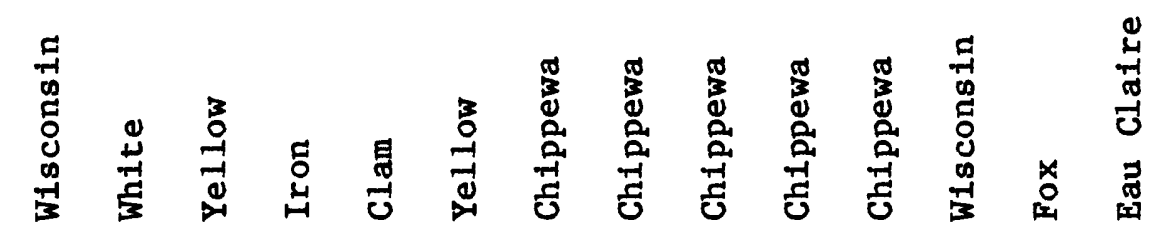

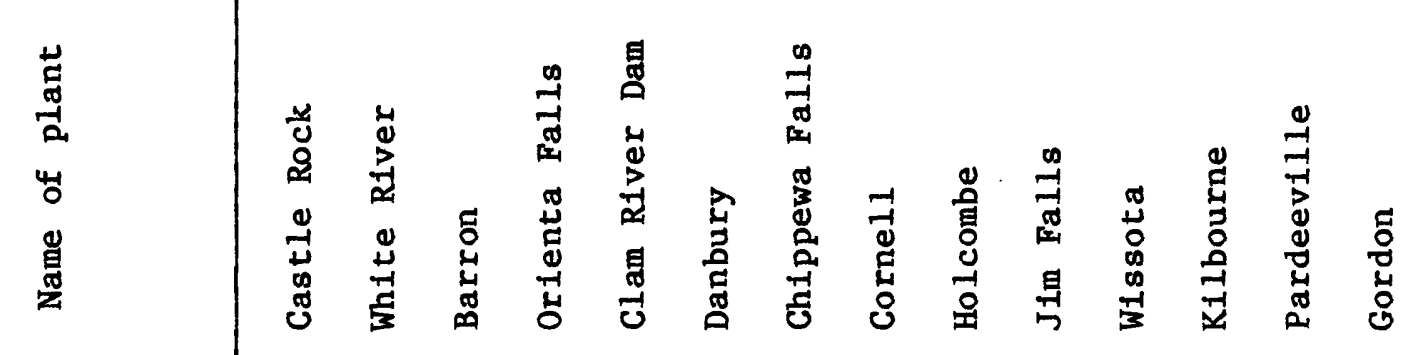

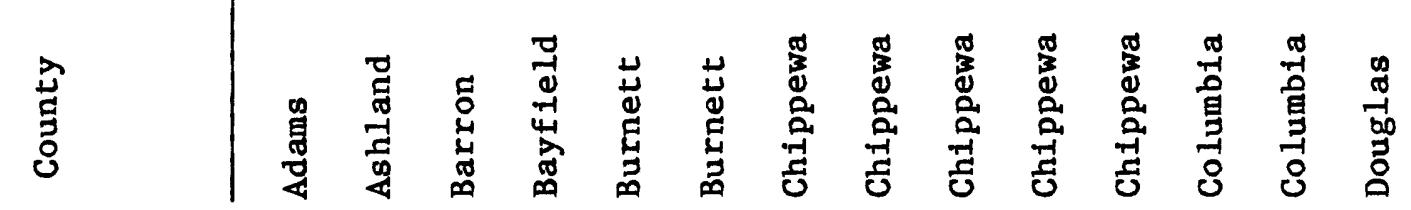




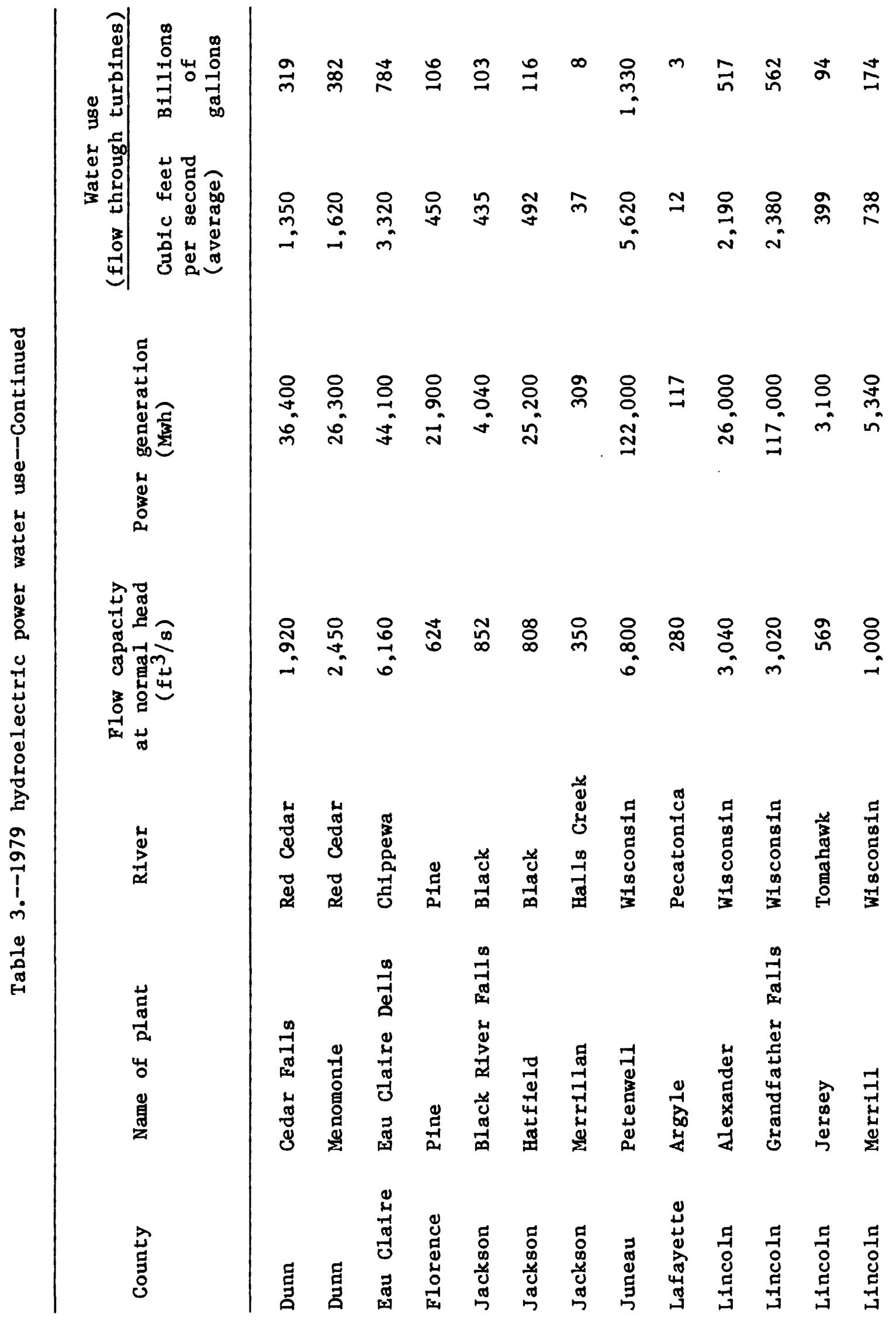




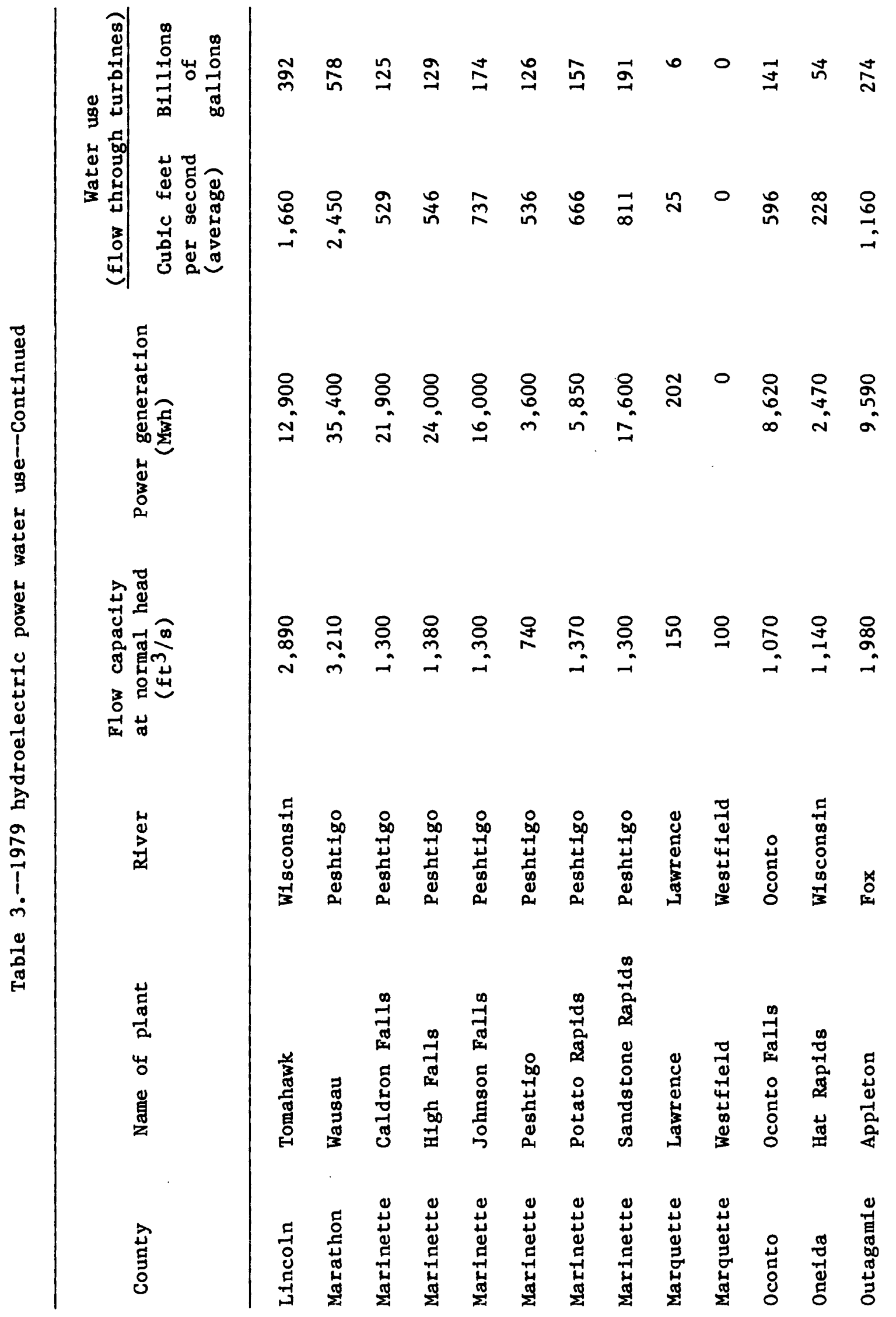




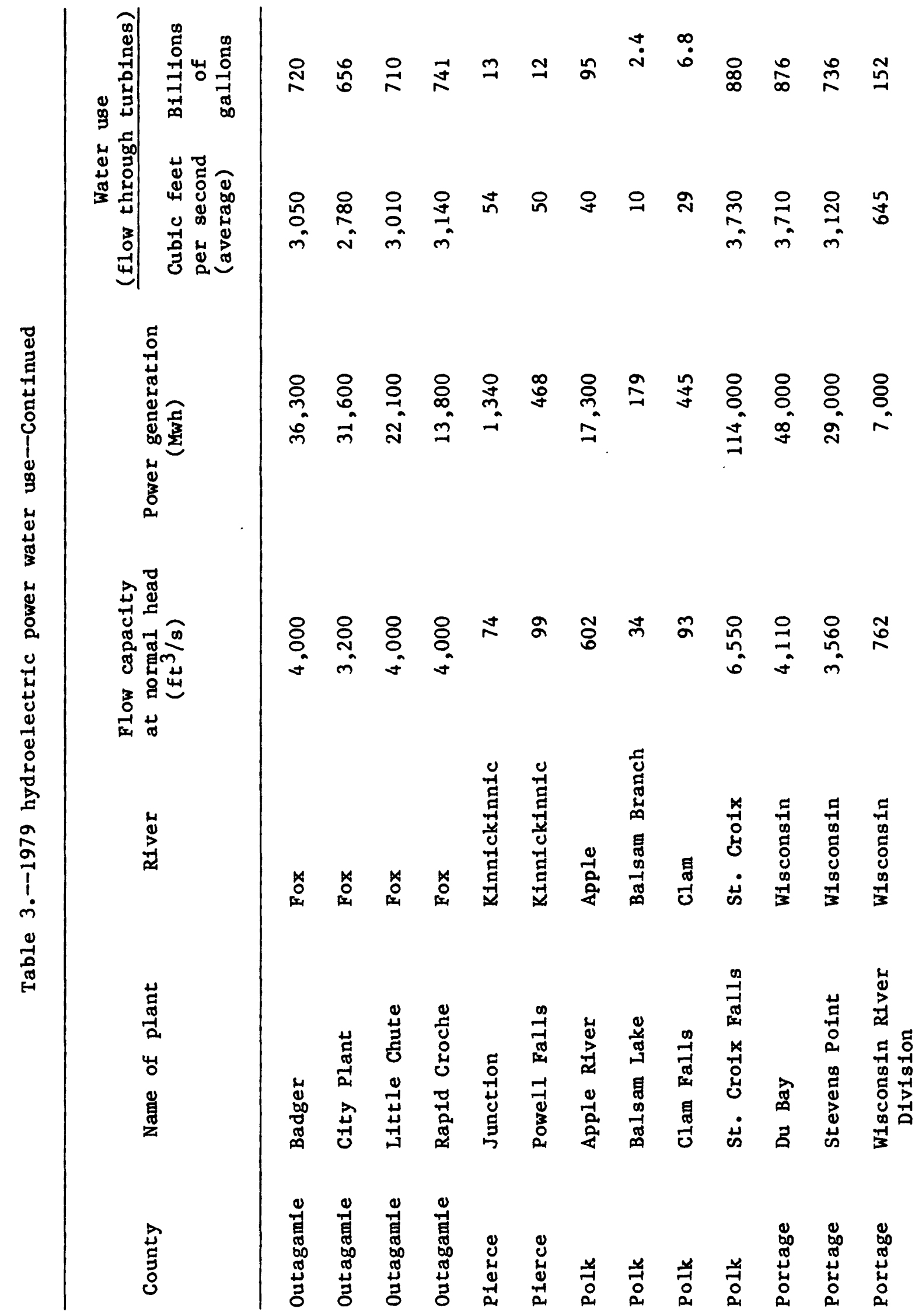




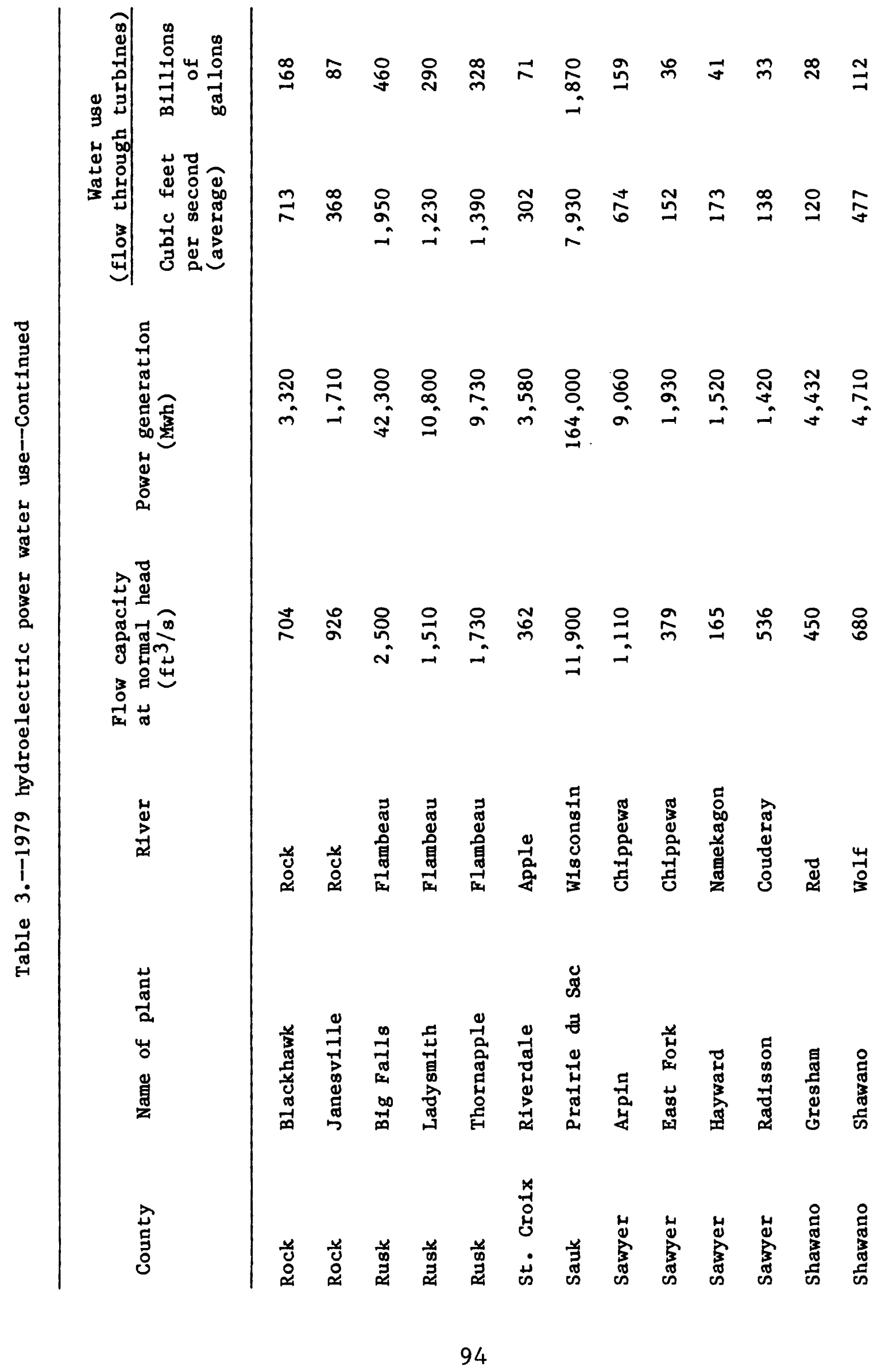




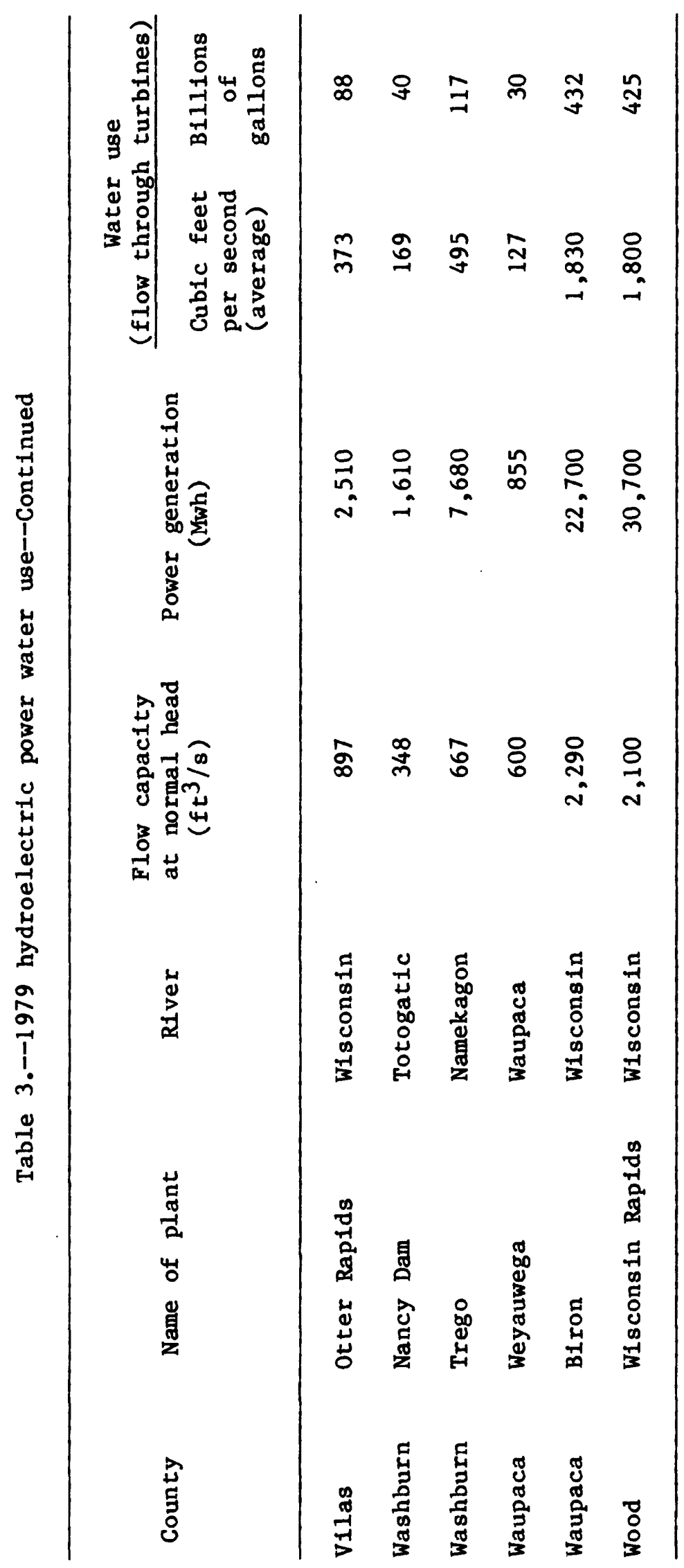




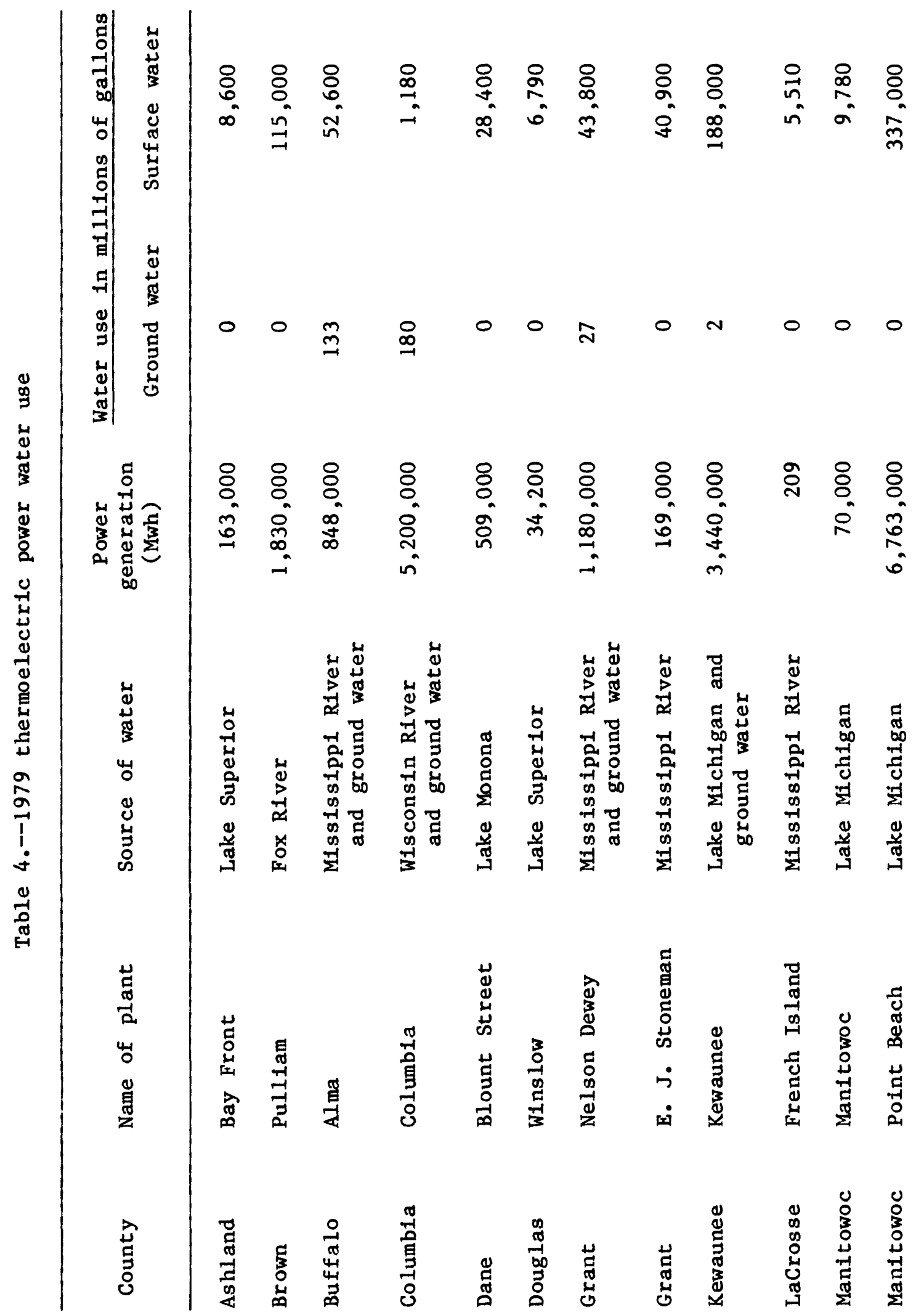




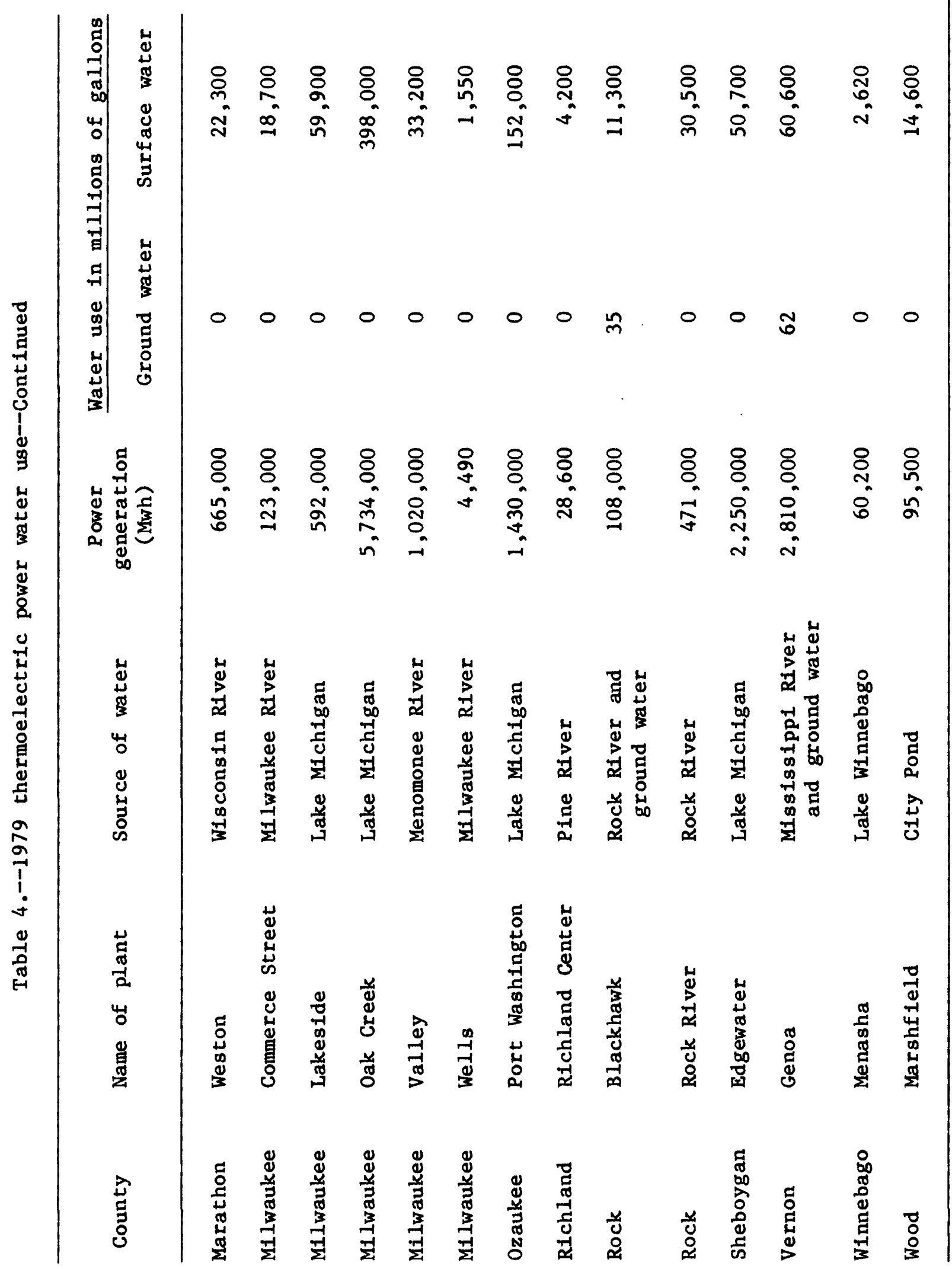


Table 5.--1979 Water Use Summary for Wisconsin, in millions of gallons

\begin{tabular}{|c|c|c|c|c|c|c|c|c|c|c|}
\hline \multirow{2}{*}{ Type of system } & \multicolumn{2}{|c|}{ Residential } & \multicolumn{2}{|c|}{ Industrial } & \multicolumn{2}{|c|}{ Commercial } & \multicolumn{2}{|c|}{ Irrigation } & \multicolumn{2}{|c|}{ Stock } \\
\hline & GW & SW & GW & SW & GW & SW & GW & SW & GW & SW \\
\hline \multicolumn{11}{|l|}{$\begin{array}{l}\text { PUBLIC } \\
\text { Community } \\
\text { systems }\end{array}$} \\
\hline Municipal & 30,600 & 32,100 & 25,000 & 37,700 & 15,200 & 15,400 & 0 & 0 & 0 & 0 \\
\hline Other & 2,910 & 0 & 143 & 0 & 1,190 & 0 & 0 & 0 & 0 & 0 \\
\hline $\begin{array}{l}\text { Noncoinmunity } \\
\text { systems }\end{array}$ & 0 & 0 & 508 & 0 & 1,870 & 0 & 0 & 0 & 0 & 0 \\
\hline PRIVATE & 26,600 & 0 & 23,000 & 128,800 & 224 & 0 & 29,600 & 1,030 & 26,700 & 1,020 \\
\hline Subtotal & 60,100 & 32,100 & 48,600 & 166,500 & 18,500 & 15,400 & 29,600 & 1,030 & 26,700 & 1,020 \\
\hline TOTAL & 92 & 200 & 215 & 100 & 33 & 900 & 30 & 600 & 27,7 & \\
\hline
\end{tabular}

\begin{tabular}{|c|c|c|c|c|c|c|c|c|}
\hline \multicolumn{9}{|l|}{$\begin{array}{l}\text { PUBLIC } \\
\text { Community } \\
\text { systems }\end{array}$} \\
\hline Municipal & 0 & 31,200 & $3,010,000$ & 26,600 & 15,700 & 97,400 & $3,142,000$ & $3,239,000$ \\
\hline Other & 195 & 154,000 & 0 & 671 & 0 & 5,100 & 154,000 & 159,100 \\
\hline $\begin{array}{l}\text { Noncommunity } \\
\text { systems }\end{array}$ & 0 & 0 & 0 & 691 & 0 & 3,070 & 0 & 3,070 \\
\hline Subtotal & 439 & $1,698,000$ & $26,110,000$ & 36,500 & 15,700 & 220,400 & $28,040,000$ & $28,260,000$ \\
\hline TO'TAL & & 698,000 & $26,110,000$ & 52,20 & & & & \\
\hline
\end{tabular}

Note: Because of rounding, totals do not necessarily equal the sum of parts. 